\title{
Equilibria of Dynamic Games with Many Players: Existence, Approximation, and Market Structure *
}

\author{
Sachin Adlakha ${ }^{\dagger} \quad$ Ramesh Johari $^{\ddagger} \quad$ Gabriel Y. Weintraub ${ }^{\S}$
}

First Version: October 2010; This Version: November, 2011

\begin{abstract}
In this paper we study stochastic dynamic games with many players; these are a fundamental model for a wide range of economic applications. The standard solution concept for such games is Markov perfect equilibrium (MPE), but it is well known that MPE computation becomes intractable as the number of players increases. We instead consider the notion of stationary equilibrium (SE), where players optimize assuming the empirical distribution of others' states remains constant at its long run average. We make two main contributions. First, we provide a rigorous justification for using SE. In particular, we provide a parsimonious collection of exogenous conditions over model primitives that guarantee existence of SE, and ensure that an appropriate approximation property to MPE holds, in a general model with possibly unbounded state spaces. Second, we draw a significant connection between the validity of SE, and market structure: under the same conditions that imply SE exist and approximates MPE well, the market becomes fragmented in the limit of many firms. To illustrate this connection, we study in detail a series of dynamic oligopoly examples. These examples show that our conditions enforce a form of "decreasing returns to larger states"; this yields fragmented industries in the limit. By contrast, violation of these conditions suggests "increasing returns to larger states" and potential market concentration. In that sense, our work uses a fully dynamic framework to also contribute to a longstanding issue in industrial organization: understanding the determinants of market structure in different industries.
\end{abstract}

\footnotetext{
${ }^{*}$ The authors are grateful for helpful conversations with Vineet Abhishek, Lanier Benkard, Peter Glynn, Andrea Goldsmith, Ben Van Roy, and Assaf Zeevi, and seminar participants at the INFORMS Annual Meeting and the Behavioral and Quantitative Game Theory Workshop. This work was supported by DARPA under the ITMANET program, and by the National Science Foundation.

${ }^{\dagger} \mathrm{S}$. Adlakha is with the Center for Mathematics of Information, California Institute of Technology, Pasadena, CA, 91125. adlakha@caltech.edu

${ }^{\ddagger}$ R. Johari is with the Department of Management Science and Engineering, Stanford University, Stanford, CA, 94305. ramesh. johariestanford.edu

${ }^{\S}$ G. Y. Weintraub is with the Columbia Business School, Columbia University, New York, NY, 10027. gweintraubecolumbia.edu
} 


\section{Introduction}

A common framework to study dynamic economic systems of interacting agents is a stochastic game, as pioneered by Shapley (1953). In a stochastic game agents' actions directly affect underlying state variables that influence their payoff. The state variables evolve according to a Markov process in discrete time, and players maximize their infinite horizon expected discounted payoff. Stochastic games provide a valuable general framework for a range of economic settings, including dynamic oligopolies-i.e., models of competition among firms over time. In particular, since the introduction of the dynamic oligopoly model of Ericson and Pakes (1995), they have been extensively used to study industry dynamics with heterogeneous firms in different applied settings (see Doraszelski and Pakes (2007) for a survey of this literature).

The standard solution concept for stochastic games is Markov perfect equilibrium (MPE) (Fudenberg and Tirole 1991), where a player's equilibrium strategy depends on the current state of all players. MPE presents two significant obstacles as an analytical tool, particularly as the number of players grows large. First is computability: the state space expands in dimension with the number of players, and thus the "curse of dimensionality" kicks in, making computation of MPE infeasible in many problems of practical interest. Second is plausibility: as the number of players grows large, it becomes increasingly difficult to believe that individual players track the exact behavior of the other agents.

To overcome these difficulties, previous research has considered an asymptotic regime in which the number of agents is infinite (Jovanovic and Rosenthal 1988, Hopenhayn 1992). In this case, individuals take a simpler view of the world: they postulate that fluctuations in the empirical distribution of other players' states have "averaged out" due to a law of large numbers, and thus they optimize holding the state distribution of other players fixed. Based on this insight, this approach considers an equilibrium concept where agents optimize only with respect to the long run average of the distribution of other players' states; Hopenhayn (1992) refers to this concept as stationary equilibrium (SE), and we adopt his terminology. SE are much simpler to compute and analyze than MPE, making this a useful approach across a wide range of applications. In particular, SE of infinite models have also been extensively used to study industry dynamics (see, for example, Luttmer 2007, Melitz 2003, Klette and Kortum 2004, and Hopenhayn and Rogerson 1993).

In this paper, we address two significant questions. First, under what conditions is it justifiable to use SE as a modeling tool? We provide theoretical foundations for the use of SE. In particular, our main results provide a parsimonious collection of exogenous conditions over model primitives that guarantee existence of SE, and ensure that an appropriate approximation property holds. These results provide a rigorous justification for using SE of infinite models to study stochastic games with a large but finite number of players.

The second question we address relates to a longstanding topic of research in industrial organization: when do industries fragment, and when do they concentrate? In a fragmented industry all firms have small market shares, with no single firm or group of firms becoming dominant. By contrast, in a concentrated industry, few participants that hold a notable market share can exert significant market power. In dynamic oligopoly models in particular, this is a challenging question to answer due to the inherent complexity of

MPE. Our second main contribution is to draw a significant connection between the validity of SE, and 
market structure: under the same conditions that imply SE exist and an appropriate approximation property holds, the market becomes fragmented in the limit of many firms. In particular, we interpret our conditions over model primitives as enforcement of a form of "decreasing returns to larger states" for an individual firm, that yields fragmented industries in the limit. By contrast, as we discuss, violation of these conditions suggests "increasing returns to larger states" and potential market concentration.

Our main results are described in detail below.

1. Theoretical foundations for SE: Existence of SE. We provide natural conditions over model primitives that guarantee existence of SE over unbounded state spaces. This is distinct from prior work on SE, which typically studies models with compact state spaces. Crucially, considering unbounded state spaces allows us to obtain sharp distinctions between increasing and decreasing returns to higher states, and the resulting concentration or fragmentation of an industry.

In addition, even though SE of a given model may exist over any compact state space, it may fail to exist over an unbounded state space. The reason is that agents may have incentives to grow unboundedly large and in this case the steady-state distribution is not well defined. Hence, a key aspect of our conditions is that they ensure the stability of the stochastic process that describes each agent's state evolution, and that the resulting steady-state distribution is well defined. In this way, we guarantee the compactness of an appropriately defined "best-response" correspondence. Our conditions also ensure the continuity and convexity of this correspondence, allowing us to use a topological fixed-point approach to prove existence.

2. Theoretical foundations for SE: Approximating MPE. We show that the same conditions over model primitives that ensure the existence of SE, imply that SE of infinite models approximate well MPE of models with a finite number of players, as the number of agents increases. An important condition that is required for this approximation result to hold is that the distribution of players' states in the SE under consideration must possess a light-tail, as originally observed in Weintraub et al. (2008) for a sequence of finite games, and in Weintraub et al. (2011) for a limiting infinite model like the one studied in this paper. In a light-tailed equilibrium, no single agent is "dominant;" without such a condition it is not possible for agents' to rationally ignore the state fluctuations of their dominant competitors.

Crucially, the light-tail assumption as used in Weintraub et al. (2008) and Weintraub et al. (2011) is an endogenous condition on the equilibrium outcome. A central contribution of this work is to develop exogenous conditions over model primitives that ensure the existence of light-tailed SE. In fact, the conditions that guarantee compactness in the existence result ensure that all SE are light-tailed. Thus approximation need not be verified separately; verification of our conditions simultaneously guarantees existence of SE as well as a good approximation to MPE as the number of agents increases.

3. Market structure in dynamic industries. Our results provide important insights into market structure in dynamic industries. The literature on dynamic oligopoly models has largely study individual industries in which market outcomes are very sensitive to certain model features and parameters (Doraszel- 
ski and Pakes 2007). In contrast, our results provide conditions for which we can predict important features of the equilibrium market structure for a broad range of parameters and specifications.

In particular, our conditions over model primitives imply that all SE are light-tailed, and therefore, in all SE the industry yields a fragmented market structure and no dominant firms emerge. Moreover, all these SE are valid approximations to MPE. While these conditions cannot pin-down the equilibrium exactly, they guarantee that in all of them the market structure is fragmented. In that sense, our work contributes to the "bounds approach" in the industrial organization literature pioneered by Sutton (1991), which aims to identify broad structural properties in industries that would yield a fragmented or a concentrated market structure. A novelty of our analysis compared to previous work is that it is done in a fully dynamic framework.

To illustrate the connection between our theoretical results and market structure in dynamic industries, we study in detail a collection of three examples in industrial organization. For each of these examples, we demonstrate that our conditions on model primitives that guarantee existence of light-tailed SE can be interpreted as enforcing "decreasing returns to higher states." Conversely, our analysis of the examples suggests that when these conditions are violated, the resulting models exhibit "increasing returns to higher states," and SE are not expected to provide accurate approximations or may not even exist. We note that, as emphasized above, unbounded state spaces are necessary to highlight the difference between increasing and decreasing returns to higher states.

The first example we discuss is a quality-ladder dynamic oligopoly model where firms can invest to improve a firm-specific state; e.g., a firm might invest in advertising to improve brand awareness, or invest in R\&D to improve product quality (Pakes and McGuire 1994). Firms' single period profits are determined through a monopolistic competition model. Through a limiting construction where the number of firms and market size both scale to infinity, we use our conditions to show that light-tailed SE exist and approximate MPE asymptotically if the single period profit function exhibits diminishing marginal returns to higher quality.

Next, we discuss a model with positive spillovers between firms (Griliches 1998). Here our conditions impose a form of decreasing returns in the spillover effect that, together with the decreasing returns to investment condition introduced in the previous model, ensure SE exist and provide good approximations to MPE. When the spillover effect is controlled in this way, the market is more likely to fragment.

Finally, we discuss a dynamic oligopoly that incorporates "learning-by-doing", so that firms become more efficient as they gain experience in the marketplace (Fudenberg and Tirole 1983). In this case, we find that firms' learning processes must exhibit decreasing returns to scale to ensure existence of light-tailed SE. These conditions are consistent with prior observations in the literature that suggest industries with prominent learning-by-doing effects will tend to concentrate; our results compactly quantify such intuition.

Indeed, in all these examples, our results validate intuition by providing quantifiable insight into market structure. Industries with increasing returns are typically concentrated and dominated by few firms, so SE would not be good approximations. By contrast, our conditions on model primitives delineate a broad range of industries with decreasing returns that become fragmented in the limit and for which SE provide accurate approximations. 
The remainder of the paper is organized as follows. Section 2 describes related literature. Section 3 introduces our stochastic game model, and there we define both MPE and SE. We then preview our results and discuss the motivating examples above in detail in Section 4. In Section 5, we develop exogenous conditions over model primitives that ensure existence of light-tailed SE. In Section 6, we show that under our conditions any light-tailed SE approximates MPE asymptotically. Section 7 revisits the examples in light of the theoretical results provided in the two previous sections. We conclude and discuss future research directions in Section 8. The appendices contain all mathematical proofs as well as important complementary material.

\section{Related Work}

Our work is related to previous literature that studies stationary equilibria or closely-related equilibrium concepts. SE is sometimes called mean field equilibrium because of its relationship to mean field models in physics, where large systems exhibit macroscopic behavior that is considerably more tractable than their microscopic description. (See, e.g., Blume (1993) and Morris (2000) for related ideas applied to static games.) In the context of stochastic games, SE and related approaches have been proposed under a variety of monikers across economics and engineering; see, e.g., studies of anonymous sequential games (Jovanovic and Rosenthal 1988, Bergin and Bernhardt 1995); dynamic stochastic general equilibrium in macroeconomic modeling (Stokey et al. 1989); Nash certainty equivalent control (Huang et al. 2006, 2007); mean field games (Lasry and Lions 2007); and dynamic user equilibrium (Friesz et al. 1993). SE has also been studied in recent works on information percolation models (Duffie et al. 2009), sensitivity analysis in aggregate games (Acemoglu and Jensen 2009), coupling of oscillators (Yin et al. 2010), scaling behavior of markets (Bodoh-Creed 2011), and in analysis of stochastic games with complementarities (Adlakha and Johari 2010).

Prior work has considered existence of equilibrium in stochastic games in general, but these are typically established only in restricted classes such as zero-sum games and games of identical interest; see Mertens et al. (1994) for background. Doraszelski and Satterthwaite (2010) and Escobar (2008) show existence of MPE for different classes of stochastic games under appropriate concavity assumptions. Our work is particularly related to Jovanovic and Rosenthal (1988) and Hopenhayn (1992) that consider existence of SE. The former paper considers a model similar to ours but restricts attention to compact sets, while the latter paper is focused on a specific model of oligopoly competition. Adlakha and Johari (2010) also consider

existence of SE; they focus on games with strategic complementarities, and establish existence using a constructive approach based on lattice theoretic methods. The preceding three papers study a different setting to ours and do not establish an approximation theorem. Several prior papers have considered various notions of approximation properties for SE in specific settings, either with bounded state spaces (Glynn 2004, Tembine et al. 2009, Bodoh-Creed 2011) or with an exogenous compactness assumption (Adlakha et al. 2010), or in linear-quadratic payoff models (Huang et al. 2007, Adlakha et al. 2008).

We briefly discuss here relation to our own prior work. In our previous conference papers (Adlakha et al. 2008, 2010), we study SE in a less general model of stochastic games than this paper. Though we 
study existence of SE and an appropriate approximation property, we make an endogenous assumption of compactness; in other words, we assume the model is such that in searching for SE we can restrict attention to a compact set. As a result, those results do not relate model primitives to either validity of SE as an approximation, nor to market structure. By contrast, in this paper, we derive exogenous conditions on model primitives that guarantee compactness, existence of SE, and an appropriate approximation property. In addition, as a consequence, we are able to apply our results to derive sharp insight into market structure.

Our paper is also closely related to Weintraub et al. (2011), who study a class of industry dynamic models. They also show a result that depends endogenously on SE: if a given SE satisfies an appropriate light-tail condition, then it approximates MPE well as the number of firms grows. Our paper provides several important contributions with respect to Weintraub et al. (2011). First, we consider a more general stochastic game model that allows us, for example, to study the models with spillovers and learning-bydoing. On the other hand, we do not consider entry and exit as they do; we discuss this extension in the conclusions section. We also consider a stronger approximation property. Second, and more importantly, the light-tail condition used to prove the approximation result in Weintraub et al. (2011) is a condition over equilibrium outcomes; by contrast, we provide conditions over model primitives that guarantee all SE are light-tailed and hence approximate MPE asymptotically. As a consequence, these conditions also give sharp insight into market structure in our paper. Finally, we provide a novel result pertaining to existence of SE, particularly over unbounded state spaces. We close by noting that Weintraub et al. (2011) also consider an analog of SE called "oblivious equilibrium" (OE) in models with finitely many agents. They study the relation between $\mathrm{OE}$ and SE by analyzing the hemicontinuity of the OE correspondence at the point where number of firms becomes infinite.

\section{Preliminaries and Definitions}

In this section we define our general model of a stochastic game, and proceed to define two equilibrium concepts: Markov perfect equilibrium (MPE) and stationary equilibrium (SE). We conclude by defining the asymptotic Markov equilibrium property, which requires that SE approximates MPE well as the number of players grows large.

\subsection{Stochastic Game Model}

In this section, we describe our stochastic game model. Compared to standard stochastic games in the literature (Shapley 1953), in our model, every player has an individual state. Players are coupled through their payoffs and state transitions. A stochastic game has the following elements:

Time. The game is played in discrete time. We index time periods by $t=0,1,2, \ldots$.

Players. There are $m$ players in the game; we use $i$ to denote a particular player.

State. The state of player $i$ at time $t$ is denoted by $x_{i, t} \in \mathcal{X}$, where $\mathcal{X} \subseteq \mathbb{Z}^{d}$ is a subset of the $d$ dimensional integer lattice. We use $\boldsymbol{x}_{t}$ to denote the state of all players at time $t$ and $\boldsymbol{x}_{-i, t}$ to denote the state of all players except player $i$ at time $t$. For indication of how to proceed with compact but not necessarily discrete state spaces, we refer the reader to the recent independent work of Bodoh-Creed (2011). 
Action. The action taken by player $i$ at time $t$ is denoted by $a_{i, t} \in \mathcal{A}$, where $\mathcal{A} \subseteq \mathbb{R}^{q}$ is a subset of the $q$-dimensional Euclidean space. We use $\boldsymbol{a}_{t}$ to denote the action of all players at time $t$.

Transition Probabilities. The state of a player evolves in a Markov fashion. Formally, let $h_{t}=$ $\left\{\boldsymbol{x}_{0}, \boldsymbol{a}_{0}, \ldots, \boldsymbol{x}_{t-1}, \boldsymbol{a}_{t-1}\right\}$ denote the history up to time $t$. Conditional on $h_{t}$, players' states at time $t$ are independent of each other. This assumption implies that random shocks are idiosyncratic, ruling out aggregate random shocks that are common to all players. The assumption is important to derive our asymptotic results. Player $i^{\prime} s$ state $x_{i, t}$ at time $t$ depends on the past history $h_{t}$ only through the state of player $i$ at time $t-1, x_{i, t-1}$; the states of other players at time $t-1, \boldsymbol{x}_{-i, t-1}$; and the action taken by player $i$ at time $t-1$, $a_{i, t-1}$. We represent the distribution of the next state as a transition kernel $\mathbf{P}$, where:

$$
\mathbf{P}\left(x_{i}^{\prime} \mid x_{i}, a_{i}, \boldsymbol{x}_{-i}\right)=\operatorname{Prob}\left(x_{i, t+1}=x_{i}^{\prime} \mid x_{i, t}=x_{i}, a_{i, t}=a_{i}, \boldsymbol{x}_{-i, t}=\boldsymbol{x}_{-i}\right) .
$$

Payoff. In a given time period, if the state of player $i$ is $x_{i}$, the state of other players is $\boldsymbol{x}_{-i}$, and the action taken by player $i$ is $a_{i}$, then the single period payoff to player $i$ is $\pi\left(x_{i}, a_{i}, \boldsymbol{x}_{-i}\right) \in \mathbb{R}$.

Discount Factor. The players discount their future payoff by a discount factor $0<\beta<1$. Thus a player $i$ 's infinite horizon payoff is given by: $\sum_{t=0}^{\infty} \beta^{t} \pi\left(x_{i, t}, a_{i, t}, \boldsymbol{x}_{-i, t}\right)$.

In a variety of games, coupling between players is independent of the identity of the players. The notion of anonymity captures scenarios where the interaction between players is via aggregate information about the state (e.g., see Jovanovic and Rosenthal 1988). Let $\boldsymbol{f}_{-i, t}^{(m)}(y)$ denote the fraction of players (excluding player $i$ ) that have their state as $y$ at time $t$, i.e.:

$$
\boldsymbol{f}_{-i, t}^{(m)}(y)=\frac{1}{m-1} \sum_{j \neq i} \mathbf{1}_{\left\{x_{j, t}=y\right\}},
$$

where $\mathbf{1}_{\left\{x_{j, t}=y\right\}}$ is the indicator function that the state of player $j$ at time $t$ is $y$. We refer to $\boldsymbol{f}_{-i, t}^{(m)}$ as the population state at time $t$ (from player $i$ 's point of view).

Definition 1 (Anonymous Stochastic Game). A stochastic game is called an anonymous stochastic game if the payoff function $\pi\left(x_{i, t}, a_{i, t}, \boldsymbol{x}_{-i, t}\right)$ and transition kernel $\mathbf{P}\left(x_{i, t}^{\prime} \mid x_{i, t}, a_{i, t}, \boldsymbol{x}_{-i, t}\right)$ depend on $\boldsymbol{x}_{-i, t}$ only through $\boldsymbol{f}_{-i, t}^{(m)}$. In an abuse of notation, we write $\pi\left(x_{i, t}, a_{i, t}, \boldsymbol{f}_{-i, t}^{(m)}\right)$ for the payoff to player $i$, and $\mathbf{P}\left(x_{i, t}^{\prime} \mid x_{i, t}, a_{i, t}, \boldsymbol{f}_{-i, t}^{(m)}\right)$ for the transition kernel for player $i$.

For the remainder of the paper, we focus our attention on anonymous stochastic games. For ease of notation, we often drop the subscript $i$ and $t$ and denote a generic transition kernel by $\mathbf{P}(\cdot \mid x, a, f)$, and a generic payoff function by $\pi(x, a, f)$, where $f$ represents the population state of players other than the player under consideration. Anonymity requires that a firm's single period payoff and transition kernel depend on the states of other firms via their empirical distribution over the state space, and not on their specific identify. The examples we discuss in the next section satisfy this assumption. Second, in an anonymous stochastic game the functional form of the payoff function is the same, regardless of the number of players $m$. In that sense, we often interpret the profit function $\pi(x, a, f)$ as representing a limiting regime in which the number of agents is infinite. In Section 4 we discuss how to derive this limiting profit function in different 
applications. Moreover, in Appendix B we briefly discuss how our results can be extended to include the case where there is a sequence of payoff functions that depends on the number of agents.

We introduce some additional useful notation. Let $\mathfrak{F}$ be the set of all possible population states on $\mathcal{X}$ :

$$
\mathfrak{F}=\left\{f: \mathcal{X} \rightarrow[0,1] \mid f(x) \geq 0, \sum_{x \in \mathcal{X}} f(x)=1\right\}
$$

In addition, we let $\mathfrak{F}^{(m)}$ denote the set of all population states in $\mathfrak{F}$ over $m-1$ players, i.e.:

$$
\mathfrak{F}^{(m)}=\left\{f \in \mathfrak{F}: \text { there exists } \boldsymbol{x} \in \mathcal{X}^{m-1} \text { with } f(y)=\frac{1}{m-1} \sum_{j} \mathbf{1}_{\left\{x_{j}=y\right\}}, \forall y \in \mathcal{X}\right\} \text {. }
$$

\subsection{Markov Perfect Equilibrium}

In studying stochastic games, attention is typically focused on Markov strategies, where the action of a player at each time is a function of only current state of every player (Fudenberg and Tirole 1991, Maskin and Tirole 1988). In the context of anonymous stochastic games, a Markov strategy depends on the current state of the player as well as the current population state. Because a player using such a strategy tracks the evolution of the other players, we refer to such strategies in our context as cognizant strategies.

Definition 2. Let $\mathfrak{M}$ be the set of cognizant strategies available to a player. That is, $\mathfrak{M}=\{\mu \mid \mu: \mathcal{X} \times \mathfrak{F} \rightarrow$ $\mathcal{A}\}$.

Consider an $m$-player anonymous stochastic game. At every time $t$, player $i$ chooses an action $a_{i, t}$ that depends on its current state and on the current population state $\boldsymbol{f}_{-i, t}^{(m)} \in \mathfrak{F}^{(m)}$. Letting $\mu_{i} \in \mathfrak{M}$ denote the cognizant strategy used by player $i$, we have $a_{i, t}=\mu_{i}\left(x_{i, t}, \boldsymbol{f}_{-i, t}^{(m)}\right)$. The next state of player $i$ is randomly drawn according to the kernel $\mathbf{P}$ :

$$
x_{i, t+1} \sim \mathbf{P}\left(\cdot \mid x_{i, t}, \mu_{i}\left(x_{i, t}, \boldsymbol{f}_{-i, t}^{(m)}\right), \boldsymbol{f}_{-i, t}^{(m)}\right) .
$$

We let $\boldsymbol{\mu}^{(m)}$ denote the strategy vector where every player has chosen strategy $\mu$. Define $V^{(m)}\left(x, f \mid \mu^{\prime}, \boldsymbol{\mu}^{(m-1)}\right)$ to be the expected net present value for a player with initial state $x$, and with initial population state $f \in$ $\mathfrak{F}^{(m)}$, given that the player follows a strategy $\mu^{\prime}$ and every other player follows the strategy $\mu$. In particular, we have

$$
\begin{aligned}
V^{(m)}\left(x, f \mid \mu^{\prime}, \boldsymbol{\mu}^{(m-1)}\right) & \triangleq \\
\mathbb{E} & {\left[\sum_{t=0}^{\infty} \beta^{t} \pi\left(x_{i, t}, a_{i, t}, \boldsymbol{f}_{-i, t}^{(m)}\right) \mid x_{i, 0}=x, f_{-i, 0}^{(m)}=f ; \mu_{i}=\mu^{\prime}, \boldsymbol{\mu}_{-i}=\boldsymbol{\mu}^{(m-1)}\right], }
\end{aligned}
$$

where $\boldsymbol{\mu}_{-i}$ denotes the strategies employed by every player except $i$. Note that state sequence $x_{i, t}$ and population state sequence $\boldsymbol{f}_{-i, t}^{(m)}$ evolve according to the transition dynamics (4).

We focus our attention on a symmetric Markov perfect equilibrium (MPE), where all players use the same cognizant strategy $\mu$. In an abuse of notation, we write $V^{(m)}\left(x, f \mid \boldsymbol{\mu}^{(m)}\right)$ to refer to the expected 
discounted value as given in equation (5) when every player follows the same cognizant strategy $\mu$.

Definition 3 (Markov Perfect Equilibrium). The vector of cognizant strategies $\boldsymbol{\mu}^{(m)} \in \mathfrak{M}$ is a symmetric Markov perfect equilibrium (MPE) if for all initial states $x \in \mathcal{X}$ and population states $f \in \mathfrak{F}^{(m)}$ we have $\sup _{\mu^{\prime} \in \mathfrak{M}} V^{(m)}\left(x, f \mid \mu^{\prime}, \boldsymbol{\mu}^{(m-1)}\right)=V^{(m)}\left(x, f \mid \boldsymbol{\mu}^{(m)}\right)$.

Thus, a Markov perfect equilibrium is a profile of cognizant strategies that simultaneously maximize the expected discounted payoff for every player, given the strategies of other players. ${ }^{1}$ It is a well known fact that computing a Markov perfect equilibrium for a stochastic game is computationally challenging in general (Doraszelski and Pakes 2007). This is because to find an optimal cognizant strategy, each player needs to track and forecast the exact evolution of the entire population state. In certain scenarios, it might be infeasible to exchange or learn this information at every step because of limited communication capacity between players or limited cognitive ability. Moreover, even if this is possible, the computation of an optimal cognizant strategy is subject to a curse of dimensionality; the state space $\mathfrak{F}^{(m)}$ grows too quickly as the number of agents $m$ and/or the number of individual states $\mathcal{X}$ becomes large. As a consequence, computing Markov perfect equilibrium in practice is only possible in models with few agents and few individual states, severely restricting the set of problems for which this equilibrium concept can be used. In the next subsection, we describe a scheme for approximating Markov perfect equilibrium that alleviates these difficulties.

\subsection{Stationary Equilibrium}

In a game with a large number of players, we might expect that fluctuations of players' states "average out" and hence the actual population state remains roughly constant over time. Because the effect of other players on a single player's payoff and transition probabilities is only via the population state, it is intuitive that, as the number of players increases, a single player has negligible effect on the outcome of the game. Based on this intuition, related schemes for approximating MPE have been proposed in different application domains via a solution concept we call stationary equilibrium or SE (see Sections 1 and 2 for references on SE and related work).

We consider a limiting model with an infinite number of agents in which a law of large numbers holds exactly. In an SE of this model, each player optimizes its payoff assuming the population state is fixed at its long-run average. Thus, rather than keep track of the exact population state, a single player's immediate action depends only on his own current state. We call such players oblivious, and refer to their strategies as oblivious strategies. (This terminology is due to Weintraub et al. 2008.) Formally, we let $\mathfrak{M}_{O}$ denote the set of (stationary, nonrandomized) oblivious strategies, defined as follows.

Definition 4. Let $\mathfrak{M}_{O}$ be the set of oblivious strategies available to a player. That is, $\mathfrak{M}_{O}=\{\mu \mid \mu: \mathcal{X} \rightarrow$ $\mathcal{A}\}$.

\footnotetext{
${ }^{1}$ Under the assumptions we make later in this paper, it can be shown that for any vector of cognizant strategies of players other than $i$, an optimal cognizant strategy always exists for player $i$.
} 
Given a strategy $\mu \in \mathfrak{M}_{O}$, an oblivious player $i$ takes an action $a_{i, t}=\mu\left(x_{i, t}\right)$ at time $t$; as before, the next state of the player is randomly distributed according to the transition kernel $\mathbf{P}$ :

$$
x_{i, t+1} \sim \mathbf{P}\left(\cdot \mid x_{i, t}, \mu\left(x_{i, t}\right), f\right)
$$

Note that because we are considering a limiting model, the player's state evolves according to a transition kernel with fixed population state $f$. The interpretation is that a single player conjectures the population state to be $f$; therefore, in determining a player's future expected payoff stream, it considers a transition kernel where its own state evolution is affected by the fixed population state $f$.

We define the oblivious value function $\tilde{V}(x \mid \mu, f)$ to be the expected net present value for any oblivious player with initial state $x$, when the long run average population state is $f$, and the player uses an oblivious strategy $\mu$. We have

$$
\tilde{V}(x \mid \mu, f) \triangleq \mathbb{E}\left[\sum_{t=0}^{\infty} \beta^{t} \pi\left(x_{i, t}, a_{i, t}, f\right) \mid x_{i, 0}=x ; \mu\right] .
$$

Note that the state sequence $x_{i, t}$ is determined by the strategy $\mu$ according to the dynamics (6), where the population state is fixed at $f$. We define the optimal oblivious value function $\tilde{V}^{*}(x \mid f)$ as $\tilde{V}^{*}(x \mid f)=$ $\sup _{\mu \in \mathfrak{M}_{O}} \tilde{V}(x \mid \mu, f)$. Given a population state $f$, an oblivious player computes an optimal strategy by maximizing its oblivious value function. Note that because an oblivious player does not track the evolution of the population state and its state evolution depends only on the population state $f$, if an optimal stationary nonrandomized strategy exists, it will only be a function of the player's current state-i.e., it must be oblivious even if optimizing over cognizant strategies. We capture this optimization step via the correspondence $\mathcal{P}$ defined next.

Definition 5. The correspondence $\mathcal{P}: \mathfrak{F} \rightarrow \mathfrak{M}_{O}$ maps a distribution $f \in \mathfrak{F}$ to the set of optimal oblivious strategies for a player. That is, $\mu \in \mathcal{P}(f)$ if and only if $\tilde{V}(x \mid \mu, f)=\tilde{V}^{*}(x \mid f)$ for all $x$.

Note that $\mathcal{P}$ maps a distribution to a stationary, nonrandomized oblivious strategy. This is typically without loss of generality, since in most models of interest there always exists such an optimal strategy. We later establish that under our assumptions $\mathcal{P}(f)$ is nonempty.

Now suppose that the population state is $f$, and all players are oblivious and play using a stationary strategy $\mu$. Because of averaging effects, we expect that if the number of agents is large, then the long run population state should in fact be an invariant distribution of the Markov process on $\mathcal{X}$ that describes the evolution of an individual agent, with transition kernel (6). We capture this relationship via the correspondence $\mathcal{D}$, defined next.

Definition 6. The correspondence $\mathcal{D}: \mathfrak{M}_{O} \times \mathfrak{F} \rightarrow \mathfrak{F}$ maps the oblivious strategy $\mu$ and population state $f$ to the set of invariant distributions $\mathcal{D}(\mu, f)$ associated with the dynamics (6).

Note that the image of the correspondence $\mathcal{D}$ is empty if the strategy does not result in an invariant distribution. We later establish conditions under which $\mathcal{D}(\mu, f)$ is nonempty. In addition, while we do not impose this restriction a priori, there are many models of interest where $\mathcal{D}$ is actually a function; that is, 
for all $\mu$ and $f$ the Markov process associated with the dynamics (6) will be ergodic and admit a unique invariant distribution.

We can now define stationary equilibrium. If every agent conjectures that $f$ is the long run population state, then every agent would prefer to play an optimal oblivious strategy $\mu$. On the other hand, if every agent plays $\mu$ and the population state is in fact $f$, then we should expect the long run population state of all players to be an invariant distribution of (6). Stationary equilibrium requires a consistency condition: the equilibrium population state $f$ must in fact be an invariant distribution of the dynamics (6) under the strategy $\mu$ and the same population state $f$.

Definition 7 (Stationary Equilibrium). An oblivious strategy $\mu \in \mathfrak{M}_{O}$ and a distribution $f \in \mathfrak{F}$ constitute a stationary equilibrium (SE) if $\mu \in \mathcal{P}(f)$ and $f \in \mathcal{D}(\mu, f)$.

In the event that the Markov chain induced by $\mu$ and $f$ has multiple invariant distributions, the agents must all conjecture the population state in equilibrium to be $f$. Further, in the event that there exist multiple optimal strategies given $f$, the agents must all choose to play $\mu$. In many models of interest (such as the examples presented in Section 4), both $\mathcal{P}$ and $\mathcal{D}$ are singletons, so such problems do not arise. For later reference, we define the correspondence $\Phi: \mathfrak{F} \rightarrow \mathfrak{F}$ as follows:

$$
\Phi(f)=\mathcal{D}(\mathcal{P}(f), f) .
$$

Observe that with this definition, a pair $(\mu, f)$ is an SE if and only if $f$ is a fixed point of $\Phi, f \in \Phi(f)$, such that $\mu \in \mathcal{P}(f)$ and $f \in \mathcal{D}(\mu, f)$

\subsection{Approximation}

A central goal of this paper is to determine conditions under which SE provides a good approximation to MPE as the number of players grows large. Here we formalize the approximation property of interest, referred to as the asymptotic Markov equilibrium (AME) property. Intuitively, this property requires that a stationary equilibrium strategy is approximately optimal even when compared against Markov strategies, as the number of players grows large.

Definition 8 (Asymptotic Markov Equilibrium). A stationary equilibrium $(\mu, f)$ possesses the asymptotic Markov equilibrium (AME) property if for all states $x$ and sequences of cognizant strategies $\mu_{m} \in \mathfrak{M}$, we have:

$$
\limsup _{m \rightarrow \infty} V^{(m)}\left(x, f^{(m)} \mid \mu_{m}, \boldsymbol{\mu}^{(m-1)}\right)-V^{(m)}\left(x, f^{(m)} \mid \boldsymbol{\mu}^{(m)}\right) \leq 0,
$$

almost surely, where the initial population state $f^{(m)}$ is derived by sampling each other player's initial state independently from the probability mass function $f$.

Note that $V^{(m)}\left(x, f^{(m)} \mid \mu^{\prime}, \boldsymbol{\mu}^{(m-1)}\right)$ is the actual value function of a player as defined in equation (5), when the player uses a cognizant strategy $\mu^{\prime}$ and every other player plays an oblivious strategy $\mu$. Similarly, $V^{(m)}\left(x, f^{(m)} \mid \boldsymbol{\mu}^{(m)}\right)$ is the actual value function of a player as defined in equation (5) when every player is playing the oblivious strategy $\mu$. AME requires that the error when using the SE strategy approaches 
zero almost surely with respect to the randomness in the initial population state. Hence, AME requires that the SE strategy becomes approximately optimal as the number of agents grows, with respect to population states that have nonzero probability of occurrence when sampling individual states according to the invariant distribution. ${ }^{2}$ This definition can be shown to be stronger than the definition considered by Weintraub et al. (2008), where AME is defined only in expectation with respect to randomness in the initial population state.

\subsection{Extensions to the Basic Model}

We briefly mention two extensions for which all our results follow. These extensions are often important in applications, but do not require any significant technical arguments. See Appendix A for further details.

First, note that players are ex-ante homogeneous in the model considered, in the sense that they share the same model primitives. This is not a particularly consequential choice, and is made primarily for notational convenience; indeed, by an appropriate redefinition of state we can model agent heterogeneity via types.

Second, note that in the game defined here, players are coupled through their states: both the transition kernel and the payoff depend on the current state of all players. However, in many models of interest the transition kernel and payoff of a player may depend on both the current state and current actions of other players. In particular, the example in Section 4.3 is a model where players are coupled through their actions. All the results of this paper naturally extend to a setting where players may also be coupled through their actions, i.e., where the transition kernel and payoff may depend on the current actions of all players as well. In the context of this paper, when players are coupled through actions, for technical simplicity we focus on finite action spaces. In this setting, to ensure existence of equilibrium, we assume that players maximize payoffs with respect to randomized strategies. In addition, we briefly discuss how our results could be extended to include continuous action spaces as well (see Appendix A).

\section{Preview of Results and Motivating Examples}

As discussed in the Introduction, this paper makes two complementary contributions. On one hand, we establish sufficient conditions over model primitives that provide justification for use of SE (in particular, that guarantee existence of SE and the AME property). On the other hand, we demonstrate that our conditions encode an economic dichotomy, broadly, between "increasing" and "decreasing" returns to higher states; the latter corresponds to those models where the industry becomes fragmented in the limit and SE is an appropriate modeling tool. In this way, our conditions directly provide insight into market structure.

This section is devoted to introducing examples drawn from industrial organization that motivate and illustrate our results. Each example presents the same basic difficulty: in terms of the parameters of the model, where does the boundary lie between those markets where fragmentation might arise, and those markets where concentration might be expected? As suggested by the preceding discussion, we use SE as a tool to inform this market structure question. In each example, we discuss how our technical results yield

\footnotetext{
${ }^{2}$ As noted earlier, under the assumptions we make an optimal cognizant strategy can be shown to exist, for any vector of cognizant strategies of the opponents. Therefore the AME property can be equivalently stated as the requirement that for all $x$ : $\lim _{m \rightarrow \infty}\left(\sup _{\mu_{m} \in \mathfrak{M}} V^{(m)}\left(x, f^{(m)} \mid \mu_{m}, \boldsymbol{\mu}^{(m-1)}\right)-V^{(m)}\left(x, f^{(m)} \mid \boldsymbol{\mu}^{(m)}\right)\right)=0$, almost surely.
} 
sharp conditions under which SE exist, the AME property holds, and all SE yield market fragmentation. We also discuss how failure of the conditions would suggest market concentration.

To set the stage, we first briefly preview the approach behind our main technical results (see Section 5 and 6). The mathematical complexity in our analysis arises due to unbounded state spaces; these are essential if we hope to identify a boundary between fragmentation and concentration in the limit of many firms. Unfortunately, with unbounded state spaces, both existence of SE and the AME property may become difficult to establish. Informally, this is because mass in the population state may "escape" to larger states as the number of firms grows; alternatively, firms may choose strategies that lead to unbounded steady state distributions over the state space.

The key condition we require to overcome these hurdles is to ensure that SE have light tails, i.e., limited mass at larger states (in a sense we make precise later). We develop exogenous conditions over model primitives that ensure all SE population states have light tails, and we further show that all light-tailed SE satisfy the AME property (extending a prior result of Weintraub et al. (2011)). Light tails ensure that no single dominant agent emerges in the limit of many firms. Note that in market structure terms, this is exactly market fragmentation.

Interpretation of our exogenous conditions reveals exactly the dichotomy introduced above: the conditions enforce a form of "decreasing returns to higher states" in the optimization problem faced by an individual agent, while their failure corresponds roughly to "increasing returns." Notably, all our results in the examples are simply applications of the same theoretical architecture. As we point out, when the examples below violate the assumptions we require-in particular, in models that exhibit increasing returns to higher states-we also expect that SE will not satisfy the AME property, and indeed, may not exist. Thus despite the fact that we only discuss sufficient conditions for existence and approximation in this paper, the examples suggest that perhaps these sufficient conditions identify a reasonable boundary between those models that admit analysis via SE, and those that do not.

For the rest of this section, we consider stochastic games with $m$ players in which the state of a player takes values on $\mathbb{Z}_{+}$.

\subsection{Dynamic Oligopoly Models}

Dynamic oligopoly models have received significant attention in the recent industrial organization literature (see Doraszelski and Pakes 2007 for a survey). In these models, firms' states correspond to some variable that affects profitability; for example, the state could represent the firm's product quality, its current productivity level, or its capacity. Per period profits are based on a static competition game, with heterogeneity among firms determined by their respective quality levels. Firms take actions to improve their quality; in the absence of this investment quality degrades over time.

Such models are extremely broad and capture a wide range of dynamic phenomena in industrial organization. In this context, we address the following important question: under what conditions on the model

primitives do we obtain concentration of the market, and under what conditions do we obtain fragmentation? Intuitively, we might expect that firms need to exhibit decreasing returns to their investments to obtain fragmentation. Our technical results yield a simple condition on model primitives that formalizes this intuition: 
we require that the single stage profit function exhibits decreasing returns to firm quality. In this case SE exist, the AME property holds, and the market structure is fragmented in the limit.

We now describe our specific model and our result in more detail.

States. For concreteness, here we consider the quality ladder model of Pakes and McGuire (1994), where the state $x_{i, t} \in \mathbb{Z}_{+}$represents the quality of the product produced by firm $i$ at time $t$.

Actions. Investments improve the state variable over time. At each time $t$, firm $i$ invests $a_{i, t} \in[0, \bar{a}]$ to improve the quality of its product. The action changes the state of the firm in a stochastic fashion as described below.

Payoffs. We consider a payoff function derived from price competition under a classic logit demand system. In such a model, there are $n$ consumers in the market. In period $t$, consumer $j$ receives utility $u_{i j t}$ from consuming the good produced by firm $i$ given by: $u_{i j t}=\theta_{1} \ln \left(x_{i t}+1\right)+\theta_{2} \ln \left(Y-p_{i t}\right)+\nu_{i j t}$, where $\theta_{1}, \theta_{2}>0, Y$ is the consumer's income, and $p_{i t}$ is the price of the good produced by firm $i$. Here $\nu_{i j t}$ are i.i.d. Gumbel random variables that represent unobserved characteristics for each consumer-good pair.

We assume that there are $m$ firms that set prices in the spot market. For a constant marginal production $\operatorname{cost} c$, there is a unique Nash equilibrium in pure strategies of the pricing game, denoted $p_{t}^{*}$ (Caplin and Nalebuff 1991). For our limit profit function, we consider an asymptotic regime in which the market size $n$ and the number of firms $m$ grow to infinity at the same rate. The limiting profit function corresponds to a logit model of monopolistic competition (Besanko et al. 1990) and is given by $\pi(x, a, f)=\frac{\tilde{c}(x+1)^{\theta_{1}}}{\sum_{y} f(y)(y+1)^{\theta_{1}}}-$ $d a$, where $\tilde{c}$ is a constant that depends on the limit equilibrium price, $c, \theta_{2}$, and $Y$. Here the second term is the cost of investment, where $d>0$ is the marginal cost per unit investment.

Transition dynamics. We use dynamics similar to those in Pakes and McGuire (1994) that have been widely used in dynamic oligopoly models. Compared to that paper, we assume random shocks are idiosyncratic. At each time period, a firm's investment of $a$ is successful with probability $\frac{\alpha a}{1+\alpha a}$ for some $\alpha>0$, in which case the quality level of its product increases by one level. The parameter $\alpha$ represents the effectiveness of the investment. The firm's product depreciates one quality level with probability $\delta \in(0,1)$ independently at each time period. Thus a firm's state decreases by one with probability $\frac{\delta}{1+\alpha a}$; it increases by one with probability $\frac{(1-\delta) \alpha a}{1+\alpha a}$ and stays at the same level with probability $\frac{1-\delta+\delta \alpha a}{1+\alpha a}$.

Discussion. Our main result for this model is the following proposition. The proof can be found in Section 7.1.

Proposition 1. Suppose that $\theta_{1}<1$. Then there exists an $S E$ for the dynamic oligopoly model, and all $S E$ possess the AME property.

The preceding result has a natural interpretation in terms of increasing and decreasing returns to higher states. Recall that $\theta_{1}$ represents how much consumers value the quality of the products, and hence if $\theta_{1}<1$, firms have strictly decreasing marginal returns in their payoff from increasing their own state. This implies that as their state grows, firms have less incentives to invest in improving their own state and ensures that, in equilibrium, the distribution of firms over the state space has a light tail and, therefore, the market structure becomes fragmented in the limit. On the other hand, if $\theta_{1} \geq 1$, then firms have an increasing marginal gain in their payoff from increasing their own state. Because the marginal cost of investment is constant, firms may continue to invest large amounts to improve their state even at very large states. Thus, a single firm 
optimization problem may not even induce a stable Markov process, and hence an SE may not exist (and the AME property may fail).

This result matches our intuition for exactly those regimes where SE work well as approximations to equilibria in finite models. In industries with decreasing returns, we expect to see a fragmented structure in the limit. By contrast, in industries with increasing returns, market concentration would likely result in the limit, i.e., a few firms capture most of the demand in the market. This is precisely where the AME property ceases to hold.

\subsection{Dynamic Oligopoly Models with Positive Spillovers}

In this section, we extend the previous model to account for positive spillovers, or externalities, across firms. Spillovers are commonly observed in industry data and could arise, for example, due to laggard firms imitating leaders' R\&D activities (Griliches 1998). The main difference from the preceding model is that now transition dynamics are coupled among the firms: one firm's state is more likely to increase if other firms are at higher quality levels.

Again, we are led to consider the effect of spillovers on market structure. From a technical standpoint, the main complexity is that firms' best responses may lead to unbounded distributions over the state space, due to the spillover effect. Thus, in order to ensure existence of SE and the AME property, we need a condition that controls the spillover effect: intuitively, if the spillover effect is not "too strong", then the dynamics will effectively exhibit decreasing returns. Our results quantify this sufficient condition. As before, in this case, market fragmentation is obtained in the limit of many firms.

To introduce spillovers, we consider a formal model in which the state space, action space, and payoff are identical to the previous section, and we continue to use the same notation. However, we modify the transition kernel to include spillovers, as described below.

Transition dynamics. We follow the model of Xu (2008), in which transition dynamics depend not only on the action of the firm, but also on the state of its competitors. Formally, let $s_{-i, t}^{(m)}$ be the spillover effect of the population state on player $i$ at time $t$, where: $s_{-i, t}^{(m)}=\sum_{y \in \mathcal{X}} \boldsymbol{f}_{-i, t}^{(m)}(y) h_{i, t}(y)$. Here $h_{i, t}(y)$ is a weight function that distinguishes the effect of different states. For this example, we use $h_{i, t}(y)=\zeta(y) \mathbf{1}_{\left\{y>x_{i, t}\right\}}$ for some uniformly bounded function $\zeta(y)$. In this case, a firm is affected with spillovers only from firms that have a better state than its own, which seems natural. We define the effective investment of player $i$ at time $t$ by: $a_{i, t}+\gamma s_{-i, t}^{(m)} \triangleq e_{i, t}$. The constant $\gamma$ is a spillover coefficient and it captures the effect of industry state on the state transition. A higher value of $\gamma$ means a higher spillover effect. With an effective investment of $e$, similar to Section 4.1, a firm's state increases by one level with probability $\frac{\alpha e}{1+\alpha e}$. Finally, as before, the firm's product depreciates in quality by one level with probability $\delta \in(0,1)$ independently at each time period.

Discussion. Since the kernel now depends on the population state $f$ through the spillover effect, even if $\theta_{1}<1$, the population state of an agent may grow due to large competitor states. This may lead to a scenario where the image of $\Phi$ is unbounded, because firms may exhibit unbounded growth. The following proposition provides a simple condition for existence of SE. The proof can be found in Section 7.2. 
Proposition 2. Suppose that $\theta_{1}<1$, and:

$$
\gamma<\frac{\delta}{(1-\delta) \alpha \sup _{y} \zeta(y)}
$$

Then there exists an SE for the dynamic oligopoly model with spillovers, and all SE possess the AME property.

Condition (10) admits a simple interpretation. This condition enforces a form of decreasing returns in the spillover effect. If the spillover effect is too large relative to depreciation-i.e., if (10) fails-then the state of a given firm has positive drift whenever other firms have large states; and in this case we expect that, for some $f$, the spillover effect can lead to optimal oblivious strategies that yield unbounded growth. On the other hand, when (10) holds, then this effect is controlled, and despite the presence of positive spillovers the state distribution has a light tail in equilibrium and the industry becomes fragmented in the limit.

What happens when the sufficient condition fails? We present one informal scenario that suggests market concentration may result. Observe that it is plausible that if the condition fails, few firms will have enough incentives to grow large to obtain a competitive advantage. Moreover, it is also plausible that a significant fraction of "fringe" firms will remain small to free-ride on the "dominant" firms. In this sense, when our condition is violated, a dramatically different market structure might be expected.

\subsection{Learning-By-Doing}

Another example that commonly arises in oligopoly settings is learning-by-doing, where firms become more efficient by producing goods. In a learning-by-doing model, the state of the firm represents its experience level; this grows in response to production, and otherwise depreciates over time.

In this type of model, it is clear that we require a dichotomy between "increasing" and "decreasing" returns to experience. Firms have to produce even in the absence of learning, simply to earn profits in each period. Note that if experience levels continue to grow without bound, then it will be impossible to ensure SE are light tailed. We show this is in fact sufficient: as long as experience begins to depreciate at sufficiently large states (in a sense we make precise), then SE exist, the market becomes fragmented in the limit, and the AME property holds.

We now describe our model; the variant we study is inspired by Fudenberg and Tirole (1983).

States. We let the state $x_{i, t}$ represent the cumulative experience level of a firm at time $t$; this represents the knowledge accumulated through past production.

Actions. The action $a_{i, t}$ represents the firm's output (i.e., goods produced) at time $t$. We consider a model in which firms compete on quantity; thus firms are coupled to each other through their actions. As discussed in Section 3.5, such an extension can be accommodated within our framework by restricting pure actions to lie on a finite subset $S=\left\{0,1, \ldots, s_{\max }\right\}$ of the integers. ${ }^{3}$

\footnotetext{
${ }^{3}$ This amounts to discretizing the action space of production quantities. In this case, we allow for mixed strategies to ensure existence of SE (see Proposition 5). However, note that in many models of interest, under the appropriate concavity assumptions, this is not very restrictive as firms will mix between two adjacent pure actions in equilibrium.
} 
Payoffs. At each time period, firms produce goods and compete in a market with $n$ consumers. Let $P_{n}(\cdot) \geq 0$ be the inverse demand function for a market size of $n$. For state $x$, pure action $s$, and population state-action profile $f$, we can write the payoff function as $\pi_{n}(x, s, f, m)=s P_{n}\left(s+(m-1) \sum_{x^{\prime}, s^{\prime}} s^{\prime} f\left(x^{\prime}, s^{\prime}\right)\right)$ $C(x, s)$, where the argument of $P_{n}$ is the aggregate output (from $m$ firms) in the market. Note that $f$ is a distribution over state-action pairs. Here, $C(x, s)$ denotes the cost of producing quantity $s$ when the firm's experience level is $x$. We assume that $C$ is nonnegative, decreasing, and convex in $x$; is increasing and convex in $s$; and has decreasing differences between $x$ and $s$. Consider a limiting case where both the number of firms $m$ and the market size $n$ become large at the same rate. We assume that there exists a limiting decreasing continuous demand function $P$ such that the limit profit function is given by $\pi(x, s, f)=s P\left(\sum_{x^{\prime}, s^{\prime}} s^{\prime} f\left(x^{\prime}, s^{\prime}\right)\right)-C(x, s)$. Note that the limiting case represents perfect competition as firms become price takers.

Transition dynamics. A firm's cumulative experience is improved as it produces more goods since it learns from the production process. On the other hand, experience capital depreciates over time due to "organizational forgetting." We assume that a firm's experience evolves independent of the experience level or the output of other firms in the market. For concreteness, we assume the transition dynamics are the same as those described in Section 4.1.

Discussion. Let $\lim _{x \rightarrow \infty} C(x, s)=\underline{C}(s)$, that is, $\underline{C}(s)$ is the cost of producing quantity $s$ for a firm with infinite experience. Our main result for this model is the following proposition. The proof can be found in Section 7.3.

Proposition 3. Let $s^{*}$ be the production level that maximizes $s P(0)-\underline{C}(s)$. Suppose that for all sufficiently large $x$ and all actions $s \in\left[0, s^{*}\right]$, we have $\sum_{x^{\prime}} x^{\prime} \mathbf{P}\left(x^{\prime} \mid x, s\right)<x$; i.e., the state has negative drift at all such pairs $(x, s)$. Then there exists an SE for the learning-by-doing model, and all SE possess the AME property.

Observe that $s p-C(x, s)$ is the single period profit to a firm when the market price is $p$, the firm produces quantity $s$, and its experience level is $x$. Generally speaking, because of learning, firms at low experience levels face strong incentives to increase their experience, leading them to produce beyond the single period optimal quantity. However, for firms at high experience levels, the choice of optimal quantity is driven primarily by maximization of single period profit (because $C(x, s)$ is decreasing and convex in $x$ ). The quantity $s^{*}$ is an upper bound on the maximizer of single period profits, so the drift condition in the proposition ensures that at high experience levels, firms' maximization of single period profit does not continue to yield unbounded growth in the experience level. ${ }^{4}$

The condition requires that the transition kernel must exhibit sufficiently strong decreasing returns to scale; as long as the possible productivity gains induced by learning-by-doing are reduced at larger states, light-tailed SE will exist and the market becomes fragmented in the limit. However, if there are not diminishing returns to learning-by-doing, then a firm's experience level will grow without bound and hence a

\footnotetext{
${ }^{4}$ For example, consider $C(x, s)=s / x$. Then $s^{*}$ is the largest allowable pure action, hence, the condition requires that all actions have negative drift for sufficiently large experience levels. For a less restrictive case, consider $C(x, s)=s^{2} / x+s^{2} / c$. Then, $s^{*}=c P(0) / 2$, so the condition requires that all actions less than or equal to $c P(0) / 2$ eventually have negative drift.
} 
light-tailed SE may not exist. This is consistent with prior observations: an industry for which learning-bydoing is prevalent may naturally become concentrated over time (Dasgupta and Stiglitz 1988).

\section{Theory: Existence}

In this section, we study the existence of light-tailed stationary equilibria. We recall that $(\mu, f)$ is a stationary equilibrium if and only if $f$ is a fixed point of $\Phi(f)=\mathcal{D}(\mathcal{P}(f), f)$, such that $\mu \in \mathcal{P}(f)$ and $f \in \mathcal{D}(\mu, f)$. Thus our approach is to find conditions under which the correspondence $\Phi$ has a fixed point; in particular, we aim to apply Kakutani's fixed point theorem to $\Phi$ to find an SE.

Kakutani's fixed point theorem requires three essential pieces: (1) compactness of the range of $\Phi$; (2) convexity of both the domain of $\Phi$, as well as $\Phi(f)$ for each $f$; and (3) appropriate continuity properties of the operator $\Phi$. It is clear, therefore, that our analysis requires topologies on both the set of possible strategies and the set of population states. For the set of oblivious strategies $\mathfrak{M}_{O}$, we use the topology of pointwise convergence.

For the set of population states, we recall that a key concept in our analysis is that of "light-tailed" population states. To formalize this notion, for the set of population states we consider a topology induced by the 1-p norm. Given $p>0$, the 1-p-norm of a function $f: \mathcal{X} \rightarrow \mathbb{R}$ is given by $\|f\|_{1-p}=\sum_{x \in \mathcal{X}}\|x\|_{p}^{p}|f(x)|$, where $\|x\|_{p}$ is the usual $p$-norm of a vector. Let $\mathfrak{F}_{p}$ be the set of all possible population states on $\mathcal{X}$ with finite 1-p norm, i.e., $\mathfrak{F}_{p}=\left\{f \in \mathfrak{F}:\|f\|_{1-p}<\infty\right\}$. The requirement $f \in \mathfrak{F}_{p}$ imposes a light-tail condition over the population state $f$. The exponent $p$ controls the weight in the tail of the population state: distributions with finite 1- $p$-norms for larger $p$ have lighter tails. The condition essentially requires that larger states must have a small probability of occurrence under $f$. As we discussed in the context of our examples, light-tailed SE imply that the market structure becomes fragmented in the limit of a large number of firms.

We start with the following restatement of Kakutani's theorem.

Theorem 1 (Kakutani-Fan-Glicksberg). Suppose there exists a set $\mathfrak{C} \subseteq \mathfrak{F}_{p}$ such that (1) $\mathfrak{C}$ is convex and compact (in the 1-p norm), with $\Phi(\mathfrak{C}) \subset \mathfrak{C} ;(2) \Phi(f)$ is convex and nonempty for every $f \in \mathfrak{C}$; and (3) $\Phi$ has a closed graph on $\mathfrak{C}^{5}$ Then there exists a stationary equilibrium $(\mu, f)$ with $f \in \mathfrak{C}$.

In the remainder of this section, we find exogenous conditions on model primitives to ensure these requirements are met. We tackle them in reverse order. We first show that under an appropriate continuity condition, $\Phi$ has a closed graph. Next, we study conditions under which $\Phi(f)$ can be guaranteed to be convex. Finally, we provide conditions on model primitives under which there exists a compact, convex set $\mathfrak{C}$ with $\Phi(\mathfrak{F}) \subset \mathfrak{C}$. The conditions we provide suffice to guarantee that $\Phi(f)$ is nonempty for all $f \in \mathfrak{F}$. Taken together our conditions ensure existence of SE, as well as an additional stronger characterization: all SE are light-tailed, i.e., they have finite 1- $p$ norm. This fact will allow us to show that every SE satisfies the AME property in the next section.

\footnotetext{
${ }^{5} \Phi$ has a closed graph if the set $\{(f, g): g \in \Phi(f)\} \subset \mathfrak{F}_{p} \times \mathfrak{F}_{p}$ is closed (where $\mathfrak{F}_{p}$ is endowed with the 1-p norm).
} 


\subsection{Closed Graph}

In this section we develop conditions to ensure the model is appropriately "continuous." Before stating the desired assumption, we introduce one more piece of notation. Without loss of generality, we can view the state Markov process in terms of the increments from the current state. In particular, we can write $x_{i, t+1}=x_{i, t}+\xi_{i, t}$, where $\xi_{i, t}$ is a random increment distributed according to the probability mass function $\mathbf{Q}(\cdot \mid x, a, f)$ defined by $\mathbf{Q}\left(z^{\prime} \mid x, a, f\right)=\mathbf{P}\left(x+z^{\prime} \mid x, a, f\right)$. Note that $\mathbf{Q}\left(z^{\prime} \mid x, a, f\right)$ is positive for only those $z^{\prime}$ such that $x+z^{\prime} \in \mathcal{X}$. We make the following assumptions over model primitives.

Assumption 1 (Continuity). $\quad$ 1. Compact action set. The set of feasible actions for a player, denoted by $\mathcal{A}$, is compact.

2. Bounded increments. There exists $M \geq 0$ such that, for all $z$ with $\|z\|_{\infty}>M, \mathbf{Q}(z \mid x, a, f)=0$, for all $x \in \mathcal{X}, a \in \mathcal{A}$, and $f \in \mathfrak{F}$.

3. Growth rate bound. There exist constants $K$ and $n \in \mathbb{Z}_{+}$such that $\sup _{a \in \mathcal{A}, f \in \mathfrak{F}}|\pi(x, a, f)| \leq K(1+$ $\left.\|x\|_{\infty}\right)^{n}$ for every $x \in \mathcal{X}$, where $\|\cdot\|_{\infty}$ is the sup norm.

4. Payoff and kernel continuity. For each fixed $x, x^{\prime} \in \mathcal{X}$ and $f \in \mathfrak{F}$, the payoff $\pi(x, a, f)$ and the kernel $\mathbf{P}\left(x^{\prime} \mid x, a, f\right)$ are continuous in $a \in \mathcal{A}$.

In addition, for each fixed $x, x^{\prime} \in \mathcal{X}$, the payoff $\pi(x, a, f)$ and the kernel $\mathbf{P}\left(x^{\prime} \mid x, a, f\right)$ are jointly continuous in $a \in \mathcal{A}$ and $f \in \mathfrak{F}_{p}$ (where $\mathfrak{F}_{p}$ is endowed with the 1-p norm). ${ }^{6}$

The assumptions are fairly mild and are satisfied in a variety of models of interest. For example, all models in Section 4 satisfy it. The first assumption is standard. We also place a finite (but possibly large) bound on how much an agent's state can change in one period (Assumption 1.2), an assumption that is reasonably weak. The polynomial growth rate bound on the payoff is quite weak, and serves to exclude the possibility of strategies that yield infinite expected discounted payoff.

Finally, Assumption 1.4 ensures that the impact of action on payoff and transitions is continuous. It also imposes that the payoff function and transition kernel are "smooth" functions of the population state under an appropriate norm. We note that when $\mathcal{X}$ is finite, then $\|f\|_{1-p}$ induces the same topology as the standard Euclidean norm. However, when $\mathcal{X}$ is infinite, the 1-p-norm weights larger states higher than smaller states. In many applications, other players at larger states have a greater impact on the payoff; in such settings, continuity of the payoff in $f$ in the 1-p-norm naturally controls for this effect. Given a particular model, the exponent $p$ should be chosen to ensure continuity of the payoff and transition kernel. ${ }^{7}$ The following proposition establishes that the continuity assumptions embodied in Assumption 1 suffice to ensure that $\Phi$ has a closed graph.

Proposition 4. Suppose that Assumption 1 holds. Then $\Phi$ has a closed graph on $\mathfrak{F}_{p}$.

\footnotetext{
${ }^{6}$ Here we view $\mathbf{P}\left(x^{\prime} \mid x, a, f\right)$ as a real valued function of $a$ and $f$, for fixed $x, x^{\prime}$; note that since we have also assumed bounded increments, this notion of continuity is equivalent to assuming that $\mathbf{P}(\cdot \mid x, a, f)$ is jointly continuous in $a$ and $f$, for fixed $x$, with respect to the topology of weak convergence on distributions over $\mathcal{X}$.

${ }^{7}$ See Section 4 and Section 7 for concrete examples. For example, in subsection 4.1 the payoff function depends on the distribution $f$ via its $\theta_{1}$ moment so it is natural to endow the set of distributions with the 1- $p$ norm with $p=\theta_{1}$.
} 


\subsection{Convexity}

Next, we develop conditions to ensure that $\Phi(f)$ is convex. We first provide a result for mixed strategies and then a result for pure strategies.

\subsubsection{Mixed Strategies}

We start by considering a simple model, where the action set $\mathcal{A}$ is the simplex of randomized actions on a base set of finite pure actions. This setting is particularly useful when we assume players are coupled through actions (see Section 3.5). Formally, we have the following definition.

Definition 9. An anonymous stochastic game has a finite action space if there exists a finite set $S$ such that the following three conditions hold:

1. $\mathcal{A}$ consists of all probability distributions over $S: \mathcal{A}=\left\{a \geq 0: \sum_{s} a(s)=1\right\}$.

2. $\pi(x, a, f)=\sum_{s} a(s) \pi(x, s, f)$, where $\pi(x, s, f)$ is the payoff evaluated at state $x$, population state $f$, and pure action $s$.

3. $\mathbf{P}\left(x^{\prime} \mid x, a, f\right)=\sum_{s} a(s) \mathbf{P}\left(x^{\prime} \mid x, s, f\right)$, where $\mathbf{P}\left(x^{\prime} \mid x, s, f\right)$ is the kernel evaluated at states $x^{\prime}$ and $x$, population state $f$, and pure action $s$.

Essentially, the preceding definition allows inclusion of randomized strategies in our search for SE. This model inherits Nash's original approach to establishing existence of an equilibrium for static games, where randomization induces convexity on the strategy space. We show next that in any game with finite action spaces, the set $\Phi(f)$ is always convex.

Proposition 5. Suppose Assumption 1 holds. In any anonymous stochastic game with a finite action space, $\Phi(f)$ is convex for all $f \in \mathfrak{F}$.

The preceding result ensures that if randomization is allowed over a set of finite actions, then the map $\Phi$ is convex-valued. We conclude by noting that another simplification is possible when working with a finite action space. In particular, it is straightforward to show that if Assumption 1 holds for the payoff and transition kernel over all pure actions, then it also holds for the payoff and transition kernel over all mixed actions; Proposition 4 follows similarly. The proof follows in an easy manner using the linearity of the payoff and transition kernel. This is a valuable insight, since in applications it simplifies the complexity of checking the model assumptions necessary to guarantee existence of an equilibrium. We discuss a similar point in Section 5.3.

\subsubsection{Pure Strategies}

In contrast to the preceding section, many relevant applications typically require existence of equilibria in pure strategies. For such examples, we employ an approach based on the following proposition.

Proposition 6. Suppose that $\mathcal{P}(f)$ is a singleton for all $f \in \mathfrak{F}$. Then $\Phi(f)$ is convex for all $f \in \mathfrak{F}$. 
The proof is straightforward: $\mathcal{D}(\mu, f)$ is convex-valued for any fixed $\mu$ and $f$, since the set of invariant distributions for the kernel defined by $\mu$ and $f$ are identified by a collection of linear equations. Thus if $\mathcal{P}(f)$ is a singleton, then $\Phi(f)=\mathcal{D}(\mathcal{P}(f), f)$ will be convex.

We now provide two different assumptions over model primitives that guarantee that $\mathcal{P}(f)$ is a singleton, for all $f \in \mathfrak{F}$. The first assumption is a condition introduced by Doraszelski and Satterthwaite (2010) and is described in detail there. The assumption has found wide application in dynamic oligopoly models.

Assumption 2. 1. The state space is scalar, i.e., $\mathcal{X} \subseteq \mathbb{Z}_{+}$, and the action space $\mathcal{A}$ is a compact interval of the real numbers.

2. The payoff $\pi(x, a, f)$ is strictly decreasing and concave in a for fixed $x$ and $f$.

3. For all $f \in \mathfrak{F}$, the transition kernel $\mathbf{P}$ is unique investment choice (UIC) admissible: there exist functions $g_{1}, g_{2}, g_{3}$ such that $\mathbf{P}\left(x^{\prime} \mid x, a, f\right)=g_{1}(x, a, f) g_{2}\left(x^{\prime}, x, f\right)+g_{3}\left(x^{\prime}, x, f\right), \forall x^{\prime}, x, a, f$, where $g_{1}(x, a, f)$ is strictly increasing and strictly concave in a.

The preceding conditions ensure that for all population states $f$ and initial states $x$, and all continuation value functions, the maximization problem in the right hand side of Bellman's equation (cf. (16) in the Appendix) is strictly concave, or that the unique maximizer is a corner solution.

The previous assumption requires a single-dimensional state space and action space. Our next assumption imposes a different set of conditions over the payoff and the transition kernel, and allows for multidimensional state and action spaces. Before providing our second condition, we require some additional terminology. Let $S \subset \mathbb{R}^{n}$. We say that a function $g: S \rightarrow \mathbb{R}$ is nondecreasing if $g\left(x^{\prime}\right) \geq g(x)$ whenever $x^{\prime} \geq x$ (where we write $x^{\prime} \geq x$ if $x^{\prime}$ is at least as large as $x$ in every component). We say $g$ is strictly increasing if the inequality is strict. Let $\mathbf{P}_{\theta}$ be a family of probability distributions on $\mathcal{X}$ indexed by $\theta \in S$. Given a nondecreasing function $u: \mathcal{X} \rightarrow \mathbb{R}$, define $\mathbb{E}_{\theta}[u]=\sum_{x} u(x) \mathbf{P}_{\theta}(x)$. We say that $\mathbf{P}_{\theta}$ is stochastically nondecreasing in the parameter $\theta$, if $\mathbb{E}_{\theta}[u]$ is nondecreasing in $\theta$ for every nondecreasing function $u$. Similarly, we say that $\mathbf{P}_{\theta}$ is stochastically concave in the parameter $\theta$ if $\mathbb{E}_{\theta}[u]$ is a concave function of $\theta$ for every nondecreasing function $u$. We say that $\mathbf{P}_{\theta}$ is strictly stochastically concave if, in addition, $\mathbb{E}_{\theta}[u]$ is strictly concave for every strictly increasing function $u$. We have the following assumption.

Assumption 3. 1. The action set $\mathcal{A}$ is convex.

2. The payoff $\pi(x, a, f)$ is strictly increasing in $x$ for fixed a and $f$, and the kernel $\mathbf{P}(\cdot \mid x, a, f)$ is stochastically nondecreasing in $x$ for fixed $a$ and $f$.

3. The payoff is concave in a for fixed $x$ and $f$, and the kernel is stochastically concave in a for fixed $x$ and $f$, with at least one of the two strictly concave in a.

The following result shows the preceding conditions on model primitives ensure the optimal oblivious strategy is unique.

Proposition 7. Suppose Assumption 1 holds, and that at least one of Assumptions 2 or 3 holds. Then $\mathcal{P}(f)$ is a singleton, and thus $\Phi(f)$ is convex for all $f \in \mathfrak{F}$. 


\subsection{Compactness}

In this section, we provide conditions under which we can guarantee the existence of a compact, convex, nonempty set $\mathfrak{C}$ such that $\Phi(\mathfrak{F}) \subset \mathfrak{C}$. The assumptions we make are closely related to those needed to ensure that $\Phi(f)$ is nonempty. To see the relationship between these results, observe that in Lemma 2 in the Appendix, we show that under Assumption 1 an optimal oblivious strategy always exists for any $f \in \mathfrak{F}$. Thus to ensure that $\Phi(f)$ is nonempty, it suffices to show that there exists at least one strategy that possesses an invariant distribution. Our approach to demonstrating existence of an invariant distribution is based on the Foster-Lyapunov criterion Meyn and Tweedie (1993). Intuitively, this criterion checks whether the process that describes the evolution of an agent eventually has "negative" drift and in this way controls for the growth of the agent's state. This same argument also allows us to bound the moments of the invariant distribution - precisely what is needed to find the desired set $\mathfrak{C}$ that is compact in the 1- $p$ norm.

One simple condition under which $\Phi(f)$ is nonempty is that the state space is finite; any Markov chain on a finite state space possesses at least one positive recurrent class. In this case the entire set $\mathfrak{F}$ is compact in the 1-p norm. Thus we have the following result.

Proposition 8. Suppose Assumption 1 holds, and that the state space $\mathcal{X}$ is finite. Then $\Phi(f)$ is nonempty for all $f \in \mathfrak{F}$, and $\mathfrak{F}$ is compact in the 1-p norm.

We now turn our attention to the setting where the state space may be unbounded; for notational simplicity, in the remainder of the section we assume $\mathcal{X}=\mathbb{Z}_{+}^{d}$. In this case, we must make additional assumptions to control for the agent's growth; these assumptions ensure the optimal strategy does not allow the state to become transient, and also allows us to bound moments of the invariant distribution of any optimal oblivious strategy.

In the sequel we restrict attention to multiplicatively separable transition kernels, as defined below.

Definition 10. The transition kernel is multiplicatively separable if there exist transition kernels $\mathbf{P}_{1}, \ldots, \mathbf{P}_{d}$ such that for all $x, x^{\prime} \in \mathcal{X}, a \in \mathcal{A}, f \in \mathfrak{F}$, there holds $\mathbf{P}\left(x^{\prime} \mid x, a, f\right)=\prod_{\ell=1}^{d} \mathbf{P}_{\ell}\left(x_{\ell}^{\prime} \mid x, a, f\right)$. In this case we let $\mathbf{Q}_{1}, \ldots, \mathbf{Q}_{\ell}$ be the coordinatewise increment transition kernels; i.e., $\mathbf{Q}_{\ell}\left(z_{\ell} \mid x, a, f\right)=\mathbf{P}_{\ell}\left(x_{\ell}+z_{\ell} \mid x, a, f\right)$, for $z$ such that $x+z \in \mathcal{X}$.

This is a natural class of dynamics in models with multidimensional state spaces. We note that if $\mathcal{X}$ is one-dimensional, the definition is vacuous. We introduce the following assumption.

Assumption 4. 1. For all $\Delta \in \mathbb{Z}_{+}^{d}$, there holds $\lim \sup _{\|x\|_{\infty} \rightarrow \infty} \sup _{a \in \mathcal{A}, f \in \mathfrak{F}} \pi(x+\Delta, a, f)-\pi(x, a, f) \leq$ 0 .

2. The transition kernel $\mathbf{P}$ is multiplicatively separable.

3. For $\ell=1, \ldots, d, \mathbf{P}_{\ell}(\cdot \mid x, a, f)$ is stochastically nondecreasing in $x \in \mathcal{X}$ and $a \in \mathcal{A}$ for fixed $f \in \mathfrak{F}$.

4. For $\ell=1, \ldots, d$, and for each $a \in \mathcal{A}$ and $f \in \mathfrak{F}, \mathbf{Q}_{\ell}(\cdot \mid x, a, f)$ is stochastically nonincreasing in $x \in \mathcal{X}$. Further, for all $x \in \mathcal{X} \sup _{f} \sum_{z_{\ell}} z_{\ell} \mathbf{Q}_{\ell}\left(z_{\ell} \mid x, a, f\right)$ is continuous in $a$.

5. There exists a compact set $\mathcal{A}^{\prime} \subset \mathcal{A}$, a constant $K^{\prime}$, and a continuous, strictly increasing function $\kappa: \mathbb{R}_{+} \rightarrow \mathbb{R}_{+}$with $\kappa(0)=0$, such that: 
(a) For all $x \in \mathcal{X}, f \in \mathfrak{F}$, $a \notin \mathcal{A}^{\prime}$, there exists $a^{\prime} \in \mathcal{A}^{\prime}$ with $a^{\prime} \leq a$, such that $\pi\left(x, a^{\prime}, f\right)-$ $\pi(x, a, f) \geq \kappa\left(\left\|a^{\prime}-a\right\|_{\infty}\right)$.

(b) For all $\ell$, and all $x^{\prime}$ such that $x_{\ell}^{\prime} \geq K^{\prime}$, $\sup _{a^{\prime} \in \mathcal{A}^{\prime}} \sup _{f \in \mathcal{F}} \sum_{z_{\ell}} z_{\ell} \mathbf{Q}_{\ell}\left(z_{\ell} \mid x^{\prime}, a^{\prime}, f\right)<0$.

Some of the previous conditions are natural, while others impose a type of "decreasing returns to higher states." First, we discuss the former. Multiplicative separability (Assumption 4.2) is natural. The first part of Assumption 4.3 is also fairly weak. The transition kernel is stochastically nondecreasing in state in models for which the state is persistent, in the sense that a larger state today increases the chances of being at a larger state tomorrow. The transition kernel is stochastically nondecreasing in action in models where larger actions take agents to larger states.

Assumption 4.1, 4.4, and 4.5 impose a form of "decreasing returns to higher states" in the model. In particular, Assumption 4.1 ensures the marginal gain in payoff by increasing one's state becomes nonpositive as the state grows large. This assumption is used to show that for large enough states agents effectively become myopic; increasing the state further does not provide additional gains. Assumption 4.5 then implies that as the state grows large, optimal actions produce negative drift inducing a "light-tail" on any invariant distribution of the resulting optimal oblivious strategy. The set $\mathcal{A}^{\prime}$ can be understood as (essentially) the set of actions that maximize the single period payoff function. Assumption 4.5 is often natural because in many models of interest increasing the state beyond a certain point is costly and requires dynamic incentives; agents will take larger actions that induce positive drift only if they consider the future benefits of doing so.

The first part of Assumption 4.4 imposes a form of decreasing returns in the transition kernel. The second part of Assumption 4.4 will hold if, for example, the transition kernel is coordinatewise stochastically nonincreasing in $f \in \mathfrak{F}$ (with respect to the first order stochastic dominance ordering) and Assumption 1 holds. In this case $\sup _{f} \sum_{z_{\ell}} z_{\ell} \mathbf{Q}_{\ell}\left(z_{\ell} \mid x, a, f\right)=\sum_{z_{\ell}} z_{\ell} \mathbf{Q}_{\ell}\left(z_{\ell} \mid x, a, \underline{f}\right)$, where $\underline{f}$ is the distribution that places all its mass at state 0 .

Much of the difficulty in the proof of the result lies in ensuring that the tail of any invariant distribution obtained from an optimal oblivious strategy is uniformly light over the image of $\Phi$. The fact that Assumptions 4.1, 4.4, and 4.5 are uniform over $f$ are crucial for this purpose.

Under the preceding assumptions we have the following result.

Proposition 9. Suppose $\mathcal{X}=\mathbb{Z}_{+}^{d}$, and Assumptions 1 and 4 hold. Then $\Phi(f)$ is nonempty for all $f \in \mathfrak{F}$, and there exists a compact, convex, nonempty set $\mathfrak{C}$ such that $\Phi(\mathfrak{F}) \subset \mathfrak{C}$.

Note that the preceding result ensures $\Phi(f) \subset \mathfrak{C}$ for all $f \in \mathfrak{F}$.

We conclude this section with a brief comment regarding finite action spaces, cf. Definition 9. The key observation we make is that if Assumption 4 holds with respect to the pure actions-i.e., with $\mathcal{A}$ replaced by $S$-then the same result as Proposition 9 holds for mixed actions. A nearly identical argument applies to establish the result.

\subsection{Summary of Results}

The previous results can be summarized by the following corollary that imposes conditions over model primitives to guarantee the existence of a light-tailed SE. 
Corollary 1. Suppose that (1) Assumption 1 holds; (2) either the game has a finite action space, or Assumption 2 holds, or Assumption 3 holds; and (3) either the state space $\mathcal{X}$ is finite, or $\mathcal{X}=\mathbb{Z}_{+}^{d}$ and Assumption 4 holds. Then, there exists a SE, and every $S E(\mu, f)$ has $f \in \mathfrak{F}_{p}$.

As we have discussed and as one can show in the examples in Section 4, many models of interest satisfy Assumption 1 and Assumptions 2 or 3 (or, more generally, some condition that guarantees uniqueness of the optimal oblivious strategy); see Section 7. Hence, if these models have a finite state space, existence of SE follows immediately. If the state space is unbounded, the only condition that remains to be checked to guarantee existence of SE is Assumption 4. As discussed in the examples in Section 4, this condition imposes a form of "decreasing returns to higher states."

We conclude by emphasizing that under the assumptions of the existence result all SE have $f \in \mathfrak{F}_{p}$; in other words, all the resulting SE have a light-tail. In the context of our examples, as previously discussed, this implies that all SE yield a fragmented market structure. In addition, the light-tail property, together with Assumption 1, will be used in the next section to ensure that the AME property holds.

\section{Theory: Approximation}

In this section we show that under the assumptions of the preceding section, any SE $(\mu, f)$ possesses the AME property. We emphasize that the AME property is essentially a continuity property in the population state $f$. Under reasonable assumptions, we show that the time $t$ population state in the system with $m$ players, $f_{-i, t}^{(m)}$, approaches the deterministic population state $f$ in an appropriate sense almost surely for all $t$ as $m \rightarrow \infty$; in particular, this type of uniform law of large numbers will hold as long as $f$ has tails that are sufficiently light. If $f_{-i, t}^{(m)}$ approaches $f$ almost surely, then informally, if the payoff satisfies an appropriate continuity property in $f$, we should expect the AME property to hold. The remainder of the section is devoted to formalizing this argument.

Theorem 2 (AME). Suppose Assumption 1 holds. Let $(\mu, f)$ be a stationary equilibrium with $f \in \mathfrak{F}_{p}$. Then the AME property holds for $(\mu, f)$.

Observe that Assumption 1 is also required for the existence of SE that satisfy $f \in \mathfrak{F}_{p}$. In this sense, under our assumptions, the AME property is a direct consequence of existence. This relationship between existence and the AME property is a significant insight of our work.

The proof of the AME property exploits the fact that the 1-p-norm of $f$ must be finite (since $f \in \mathfrak{F}_{p}$ ) to show that $\left\|f_{-i, t}^{(m)}-f\right\|_{1-p} \rightarrow 0$ almost surely as $m \rightarrow \infty$; i.e., the population state of other players approaches $f$ almost surely under an appropriate norm. Continuity of the payoff $\pi$ in $f$, together with the growth rate bounds in Assumption 1, yields the desired result.

In practice, the light-tail condition-i.e., the requirement that $f \in \mathfrak{F}_{p}$-ensures that an agent's state rarely becomes too large under the invariant distribution $f$ associated with the dynamics (6). Weintraub et al. (2011) provide a similar result in a dynamic industry model with entry and exit. Our result, on the other hand, is more general in terms of the definition of the AME property, as well as the payoff functions and transition kernels considered. In particular, we allow for dependence of the transition kernel on the 
population state. This necessitates a significantly different proof technique, since agents' states do not evolve independently in general. We note that the light-tail condition is consequential, as it is possible to construct examples for which stationary equilibria exist, but $f \notin \mathfrak{F}_{p}$ and the AME property does not hold (Weintraub et al. 2011).

We conclude by noting that in many models of interest it is more reasonable to assume that the payoff function explicitly depends on the number of agents. To study these environments, we consider a sequence of payoff functions indexed by the number of agents, $\pi_{m}(x, a, f)$. Here, the profit function $\pi$ is a limit: $\lim _{m \rightarrow \infty} \pi_{m}(x, a, f)=\pi(x, a, f)$. (See Section 4 for concrete examples.) In this case, if the number of players is $m$, the actual expected net present value is defined with $\pi_{m}$; hence, the payoff function in the AME property depends on $m$. In Appendix B we show that under a strengthening of Assumption 1, Theorem 2 can be generalized to this setting.

\section{Examples Revisited}

In this section we revisit each of the examples presented in Section 4 and show that all the propositions for these examples are consequences of Corollary 1 and Theorem 2. This establishes the key connection in the paper between existence of SE and the AME property on one hand, and the impact of model primitives on market structure on the other hand. In particular, our conditions over model primitives imply that all SE are light-tailed, and therefore, in all SE the industry yields a fragmented market structure, and the AME property is satisfied.

Formally, recall that the conditions required to establish the main results of this paper are Assumption 1 (used to ensure continuity properties); Assumption 2 and/or 3 (used to ensure convexity of the image of $\Phi$ ); and Assumption 4 (used to ensure the existence of a compact subset $\mathfrak{C} \subset \mathfrak{F}$ such that $\Phi(\mathfrak{C}) \subset \mathfrak{C}$ ). Of these properties, continuity and convexity are typically straightforward to guarantee in each of the models we consider below. Thus we primarily focus on the role of Assumption 4.

\subsection{Dynamic Oligopoly Models}

In this section, we provide the proof of Proposition 1. Note that the payoff function depends on the distribution $f$ via its $\theta_{1}$ moment, and hence we endow the set of distributions with the topology induced by the 1-p norm with $p=\theta_{1}$. Since the payoff is continuous and nonincreasing in the $\theta_{1}$ moment of $f$, and the transition kernel is independent of $f$, it is straightforward to check that Assumption 1 holds. In addition, Doraszelski and Satterthwaite (2010) show that the transition kernel of this model satisfies Assumption 2 (it can also be shown that Assumption 3 is satisfied).

Thus the desired result is reduced determining whether Assumption 4 holds. It is straightforward to check that Assumptions 4.2-4 hold; we omit the details. Assumption 4.5 holds because positive drift is costly, as the kernel defined above exhibits depreciation; in particular, it suffices to set $\mathcal{A}^{\prime}=\{0\}$. Thus the central condition to check in this model is Assumption 4.1. This assumption holds if and only if $\theta_{1}<1$ : in this case, $\sup _{a, f} \pi(x+\Delta, a, f)-\pi(x, a, f) \rightarrow 0$ as $x \rightarrow \infty$ for all $\Delta>0$. Using Corollary 1 and Theorem 2 , the result follows. 
Thus, existence of SE and the AME property are closely tied to the parameter $\theta$ which represents how much consumers value the quality of the product. For $\theta<1$, the firms have decreasing marginal returns in their payoff from increasing their state. This ensures that the Markov process associated with a single firm optimization process is stable which in turn ensures that the range of $\Phi$ is compact. As discussed earlier, this condition leads to a natural separation between industries where we expect to see a fragmented market structure and the industries where market concentration is likely to result in the limit.

\subsection{Dynamic Oligopoly Models with Positive Spillovers}

In this section we provide the proof of Proposition 2. Assumption 1 and 2 follow as in the preceding result; the proof is omitted. Again we focus on Assumption 4. Assumption 4.1, 4.2, 4.3, and the first part of 4.4 hold as before; we omit the details. The key assumptions that we need to verify are thus the second part of Assumption 4.4, and Assumption 4.5.

Observe that the maximum possible value of the effective investment when a firm takes action $a$ is $e_{\max }(a) \triangleq a+\gamma \sup _{y} \zeta(y)$. A straightforward calculation yields:

$$
\begin{aligned}
\sup _{f} \sum_{z} z \mathbf{Q}(z \mid x, a, f) & =(1-\delta)\left(\frac{\alpha e_{\max }(a)}{1+\alpha e_{\max }(a)}\right)-\delta\left(\frac{1}{1+\alpha e_{\max }(a)}\right) \\
& =\frac{\alpha e_{\max }(a)}{1+\alpha e_{\max }(a)}-\delta .
\end{aligned}
$$

It follows from the definition of the transition kernel that the second part of Assumption 4.4 holds. In order for Assumption 4.5 to hold with $\mathcal{A}^{\prime}=\{0\}$, it follows that we need:

$$
\gamma<\frac{\delta}{(1-\delta) \alpha \sup _{y} \zeta(y)}
$$

Using Corollary 1 and Theorem 2, we conclude that the result of the proposition follows if (10) holds.

For industries with spillovers, the compactness assumptions requires that the spillover effect is not too large relative to depreciation. This along with decreasing marginal returns in the payoff ensures that the firms do not have unbounded growth in their state. As a result, the market structure becomes fragmented in the limit of a large number of firms.

\subsection{Learning-By-Doing}

In this section, we provide the proof of Proposition 3. Since $P$ is decreasing and $C(x, s)$ is decreasing in $x$, Assumption 1 follows in a straightforward manner in this model, as long as $P$ is continuous. Since this is a model with finite action spaces, the result of Proposition 5 also applies. Thus, as before, the proof is reduced to determining whether Assumption 4 holds for the given model. As in the preceding examples, it is straightforward to check that Assumptions 4.2, 4.3, and 4.4 hold. Note that $\pi(x+\Delta, s, f)-\pi(x, s, f)=$ $C(x, s)-C(x+\Delta, s)$ for all $x, s, f$ and $\Delta \geq 0$, and the action space is finite. Thus Assumption 4.1 follows since $C(x, s)$ is nonnegative, decreasing, and convex in $x$. 
Therefore, our focus turns to Assumption 4.5. Using standard supermodularity arguments, it is simple to check that any $s$ that maximizes $\pi(x, s, f)$ for some $x, f$ is contained in the interval $\left[0, s^{*}\right]$. In particular, then, suppose that for all sufficiently large $x$ and all actions $s \in\left[0, s^{*}\right]$, we have $\sum_{z} z \mathbf{Q}(z \mid x, s)<0$. Then Assumption 4.5 holds, so using Corollary 1 and Theorem 2 the result follows.

In learning-by-doing models, compactness of the image of $\Phi$ is ensured by requiring that the transition kernel exhibits decreasing returns to higher states. In other words, if the productivity gains induced by learning-by-doing are reduced at larger states, light-tailed SE will exist and the AME property will hold. As discussed earlier this is consistent with the observation that a very strong learning-by-doing effect (that persists even at large scale) will likely lead to market concentration.

\section{Conclusions}

This paper considered stationary equilibrium in dynamic games with many players. Our main results provide a parsimonious set of assumptions on the model primitives which ensure that a stationary equilibrium exists in a large variety of games. We also showed that the same set of assumptions ensure that SE yield fragmented market structures, and is a good approximation to MPE in large finite games. Through a set of examples, we illustrate that our conditions on model primitives can be naturally interpreted as enforcing "decreasing returns to higher states."

We conclude by noting several extensions that can be developed for the models described here.

1. Entry and exit. A natural extension, particularly relevant for dynamic oligopoly models, would be to consider a scenario where agents (i.e., firms) make entry and exit decisions endogenously in equilibrium. We conjecture that under some mild additional assumptions our results would extend to this setting.

2. Connections between $S E$ and oblivious equilibrium in finite models. In some contexts, particularly in empirical settings, it may be more appropriate to work over a model with a finite number of agents. In these cases, as discussed in Section B, it is possible to define an "oblivious equilibrium" for finite models (Weintraub et al. 2008). We conjecture that under some additional technical conditions over the model primitives we can prove that a sequence of OE satisfies the AME property.

3. Nonstationary equilibrium. Our focus was on SE because it is of practical interest and has received significant attention in the literature. We conjecture, however, that our results can be extended to nonstationary versions of an equilibrium concept based on averaging effects that could be used to approximate transitional short-run dynamics as oppose to long-run behavior.

We leave these directions for future research.

\section{References}

Acemoglu, D., M. K. Jensen. 2009. Aggregate comparative statics SSRN Working Paper. 
Adlakha, S., R. Johari. 2010. Mean field equilibrium in dynamic games with complementarities Submitted.

Adlakha, S., R. Johari, G. Y. Weintraub, A. Goldsmith. 2008. Oblivious equilibrium for large-scale stochastic games with unbounded costs. Proceedings of the IEEE Conference on Decision and Control. 5531-5538.

Adlakha, S., R. Johari, G. Y. Weintraub, A. Goldsmith. 2010. On oblivious equilibrium in large population stochastic games. Proceedings of the IEEE Conference on Decision and Control. 3117-3124.

Aliprantis, C. D., K. C. Border. 2006. Infinite dimensional analysis: a hitchhiker's guide. Springer Verlag.

Bergin, J., D. Bernhardt. 1995. Anonymous sequential games: existence and characterization of equilibria. Economic Theory 5(3) 461-489.

Bertsekas, Dimitri P. 2007. Dynamic Programming and Optimal Control, Volume II. Athena Scientific, Nashua, New Hampshire.

Besanko, D., M. K. Perry, R. H. Spady. 1990. The logit model of monopolistic competition: Brand diversity. The Journal of Industrial Economics 38(4) 397 - 415.

Blume, L. E. 1993. The statistical mechanics of best-response strategy revision. Games and Economic Behavior 11(2) 111-145.

Bodoh-Creed, A. 2011. Approximation of large dynamic games In preparation.

Cachon, Gérard P., M. A. Lariviere. 1999. Capacity choice and allocation: Strategic behavior and supply chain performance. Management Science 45(8) 1091-1108.

Caplin, A., B. Nalebuff. 1991. Aggregation and imperfect competition - on the existence of equilibrium. Econometrica 59(1) $25-59$.

Ching, A.T. 2010. Consumer learning and heterogeneity: Dynamics of demand for prescription drugs after patent expiration. International Journal of Industrial Organization .

Dasgupta, P., J. Stiglitz. 1988. Learning-by-doing, market structure and industrial and trade policies. Oxford Economic Papers 40 246-268.

Doraszelski, U., A. Pakes. 2007. A framework for applied dynamic analysis in IO. Handbook of Industrial Organization, Volume 3 .

Doraszelski, U., M. Satterthwaite. 2010. Computable Markov-perfect industry dynamics. RAND Journal of Economics 41(2) $215-243$.

Duffie, D., S. Malamud, G. Manso. 2009. Information percolation with equilibrium search dynamics. Econometrica 77(5) 1513-1574.

Ericson, R., A. Pakes. 1995. Markov-perfect industry dynamics: A framework for empirical work. Review of Economic Studies 62(1) 53-82.

Escobar, J. 2008. Existence of pure and behavior strategy stationary Markov equilibrium in dynamic stochastic games. Working paper, University of Chile.

Friesz, T. L., D. Bernstein, T. E. Smith, R. L. Tobin, B.W. Wie. 1993. A variational inequality formulation of the dynamic network user equilibrium problem. Operations Research 41(1) 179-191.

Fudenberg, D., J. Tirole. 1983. Learning-by-doing and market performance. Bell Journal of Economics 14(14) 552530.

Fudenberg, D., J. Tirole. 1991. Game Theory. The MIT Press.

Glynn, P. 2004. Distributed algorithms for wireless networks Presented at the Conference on Stochastic Networks, Montréal, Québec, Canada. 
Glynn, P. W., A. Zeevi. 2006. Bounding Stationary Expectations of Markov Processes. Markov processes and related topics: A Festschrift for Thomas G. Kurtz. Selected papers of the conference, Madison, WI, USA, July. 10-13.

Griliches, Z. 1998. R\&D and Productivy: The Econometric Evidence. The University of Chicago Press.

Hajek, B. 1982. Hitting-time and occupation-time bounds implied by drift analysis with applications. Advances in Applied probability 14(3) 502-525.

Hopenhayn, H., R. Rogerson. 1993. Job turnover and policy evaluation - a general equilibrium-analysis. Journal of Political Economy 101(5) 915 - 938.

Hopenhayn, H. A. 1992. Entry, exit, and firm dynamics in long run equilibrium. Econometrica 60(5) 1127-1150.

Huang, M., P. E. Caines, R. P. Malhamé. 2007. Large-population cost-coupled LQG problems with nonuniform agents: Individual-mass behavior and decentralized $\epsilon$-Nash equilibria. IEEE Transactions on Automatic Control 52(9) $1560-1571$.

Huang, M., R. P. Malhamé, P. E. Caines. 2006. Large population stochastic dynamic games: closed-loop MckeanVlasov systems and the Nash certainty equivalence principle. Communications in Information and Systems 6(3) $221-251$.

Jovanovic, B., R. W. Rosenthal. 1988. Anonymous sequential games. Journal of Mathematical Economics 17 77-87.

Kelly, Frank P. 1997. Charging and rate control for elastic traffic. European Transactions on Telecommunications 8 33-37.

Klette, T. J., S. Kortum. 2004. Innovating firms and aggregate innovation. Journal of Political Economy 112(5) 986 1018.

Lasry, J. M., P. L. Lions. 2007. Mean field games. Japanese Journal of Mathematics 2 229-260.

Luttmer, E. G. J. 2007. Selection, growth, and the size distribution of firms. Quarterly Journal of Economics 122(3) $1103-1144$.

Maskin, E., J. Tirole. 1988. A theory of dynamic oligopoly, I and II. Econometrica 56(3) 549 - 570.

Melitz, M. J. 2003. The impact of trade on intra-industry reallocations and aggregate industry productivity. Econometrica 71(6) 1695 - 1725.

Mertens, J.-F., S. Sorin, R. Zamir. 1994. Repeated games CORE Discussion Papers 9420, 9421, 9422.

Meyn, S. P., R. L. Tweedie. 1993. Markov Chains and Stochastic Stability. Springer-Verlag.

Morris, S. 2000. Contagion. Review of Economic Studies 67(1) 57-78.

Pakes, A., P. McGuire. 1994. Computing Markov-perfect Nash equilibria: Numerical implications of a dynamic differentiated product model. RAND Journal of Economics 25(4) 555-589.

Shapley, L. S. 1953. Stochastic games. Proceeding of the National Academy of Sciences 39 1095-1100.

Stokey, N. L., R. E. Lucas, Jr., E. C. Prescott. 1989. Recursive methods in economic dynamics. Harvard University Press, Cambridge, MA.

Sutton, J. 1991. Sunk Costs and Market Structure. 1st ed. MIT Press.

Sznitman, A.S. 1991. Topics in propagation of chaos. Ecole d'Eté de Probabilités de Saint-Flour XIX1989 165-251.

Tembine, H., J.-Y. Le Boudec, R. El-Azouzi, E. Altman. 2009. Mean field asymptotics of Markov decision evolutionary games and teams. Proceedings of GameNets '09. 140-150.

Weintraub, G. Y., C. L. Benkard, B. Van Roy. 2008. Markov perfect industry dynamics with many firms. Econometrica 76(6) 1375-1411.

Weintraub, G. Y., C. L. Benkard, B. Van Roy. 2011. Industry dynamics: Foundations for models with an infinite number of firms. Journal of Economic Theory 146 1965-1994. 
$\mathrm{Xu}, \mathrm{Y}$. 2008. A structural empirical model of R\&D, firm heterogeneity, and industry evolution. Working paper, NYU University.

Yin, H., P. G. Mehta, S. P. Meyn, U. V. Shanbhag. 2010. Synchronization of coupled oscillators is a game. IEEE CDC.

\section{A Extensions to the Basic Model}

\section{A.1 Heterogeneous Players}

In this section, we study anonymous stochastic games with ex-ante heterogeneous players. To represent this heterogeneity, at the beginning of the game, a player is assigned a type (denoted by $\theta$ ) that stays fixed for the entire duration of the game. For simplicity, we assume that the players' types are randomly and independently drawn out of a finite set $\Theta$ with a common prior distribution $\Gamma$. Let $\mathbf{P}(\cdot \mid x, a, f ; \theta)$ and $\pi(x, a, f ; \theta)$ denote the transition kernel and payoff of a type $\theta$ player.

To analyze a stochastic game with heterogeneous players, we define a new state as follows. Let $\hat{x}=$ $(x, \theta)$ be an extended state; if a player's extended state is $\hat{x}$, we interpret it to mean that the player is in state $x$ and has a type $\theta$. We let $\hat{\mathcal{X}}=\mathcal{X} \times \Theta$ denote the expanded state space. Let $\hat{f}$ denote a population state over the expanded state space, i.e., $\hat{f}$ is a distribution over $\hat{\mathcal{X}}$. Given $\hat{f}$, we define $F(\hat{f}) \in \mathfrak{F}$ by:

$$
F(\hat{f})(x)=\sum_{\theta} \hat{f}(x, \theta)
$$

We have the following two definitions:

$$
\begin{aligned}
\hat{\pi}(\hat{x}, a, \hat{f}) & =\pi(x, a, F(\hat{f}) ; \theta) ; \\
\hat{\mathbf{P}}\left(\hat{x}^{\prime} \mid \hat{x}, a, \hat{f}\right) & = \begin{cases}0, & \text { if } \theta^{\prime} \neq \theta ; \\
\mathbf{P}\left(x^{\prime} \mid x, a, F(\hat{f}) ; \theta\right), & \text { if } \theta^{\prime}=\theta .\end{cases}
\end{aligned}
$$

These definitions simply map the payoff and transition kernel with respect to the extended state back to the payoff and transition kernel in the original game. Now observe that in the new game defined in this way, it can be verified that if the original game satisfied Assumptions 1, 2 or 3, and 4 for each $\theta$, then the extended game satisfies the same assumptions as well. Thus all our preceding results apply even in games with heterogeneous players. Because strategies are a function of the extended state, in this case players of different types will use different strategies.

\section{A.2 Coupling Through Actions}

In the main development of this paper, we considered anonymous stochastic games where players are coupled to each other via the population state as defined in equation (2); note, in particular, that the population state gives the fraction of players at each state. As discussed in the Introduction, however, in many models of interest the transition kernel and payoff of a player may depend on both the current state and current actions 
of other players. In particular, the example in Section 4.3 is a model where players are coupled through their actions.

To formally model such a scenario, we consider an $m$ player stochastic game being played in discrete time over the infinite horizon, where again the payoff and transition kernel of a player are denoted by $\pi(x, a, f)$ and $\mathbf{P}(\cdot \mid x, a, f)$ respectively. ${ }^{8}$ However, we now assume that $f$ is a distribution over both states and actions. We refer to $f$ as the population state-action profile (to distinguish it from just the population state, which is the marginal distribution of $f$ over $\mathcal{X}$ ). For simplicity, since the prior development in this paper assumes state spaces are discrete, for the purposes of this subsection we restrict attention to a game with a finite action space $S \subset \mathbb{Z}^{k}$, cf. Definition 9; in particular, we assume that players maximize payoffs with respect to randomized strategies over $S$. Thus the population state-action profile is a distribution over $\mathcal{X} \times S$.

We again let $x_{i, t} \in \mathcal{X}$ be the state of player $i$ at time $t$, where $\mathcal{X} \subseteq \mathbb{Z}^{d}$. We let $s_{i, t} \in S$ be the (pure) action taken by player $i$ at time $t$. Let $\boldsymbol{f}_{-i, t}^{(m)}$ denote the empirical population state-action profile at time $t$ in an $m$-player game; in other words, $f_{i, t}^{(m)}(x, s)$ is the fraction of players other than $i$ at state $x$ who play $s$ at time $t$. With these definitions, $x_{i, t}$ evolves according to the transition kernel $\mathbf{P}$ as before, i.e., $x_{i, t+1} \sim \mathbf{P}\left(\cdot \mid x_{i, t}, a_{i, t}, f_{-i, t}^{(m)}\right)$.

A player acts to maximize their expected discounted payoff, as before. Note that a potential challenge here is that a player's time $t$ payoff and transition kernel depend on the actions of his competitors, which are chosen simultaneously with his own action. Thus to evaluate the time $t$ expected payoffs and transition kernel, a player must take an expectation with respect to the randomized strategies employed by his competitors.

Our first step is to extend the appropriate assumptions to this game model. Let $\mathfrak{F}$ now denote the set of all distributions over $\mathcal{X} \times S$, and let $\mathfrak{F}_{p}$ denote the set of all distributions in $\mathfrak{F}$ with finite 1 - $p$-norm as before. Assumptions 1 and 4 thus extend naturally to games with coupling through actions, with these new interpretations of $\mathfrak{F}$ and $\mathfrak{F}_{p}$.

The AME property continues to hold for games with coupling through actions. Recall that in the proof of Theorem 2, we establish that if $(\mu, f)$ is a stationary equilibrium, then $\left\|f_{-i, t}^{(m)}-f\right\|_{1-p} \rightarrow 0$ almost surely for all $t$, if players' initial states are sampled independently from $f$, all players other than $i$ follow strategy $\mu$, and player $i$ follows any strategy. (See Lemma 10 in the Appendix.) In a game with coupling through actions, $f_{-i, t}^{(m)}$ also tracks the empirical distribution of players' actions. However, since all players other than $i$ use the same oblivious strategy $\mu$, and since the base action space $S$ is finite, it is straightforward to extend the argument of Lemma 10 to the current setting. The remainder of the existing proof of Theorem 2 carries over essentially unchanged under Assumption 1; for brevity we omit the details.

Next, recall that to prove existence of a stationary equilibrium, we consider two maps: $\mathcal{P}(f)$ (which identifies the set of optimal oblivious strategies given $f$ ), and $\mathcal{D}(\mu, f)$ (which identifies the set of invariant distributions of the Markov process induced by $\mu$ and $f$ ). The analysis of $\mathcal{P}(f)$ proceeds exactly as before (but with randomized strategies, as discussed in Section 5.2.1). However, in a game with coupling through actions, we redefine $\mathcal{D}(\mu, f)$ to be the set of invariant distributions over $\mathcal{X} \times S$ induced by $\mu$ and $f$. In other

\footnotetext{
${ }^{8}$ For the purposes of this subsection we assume players are homogeneous.
} 
words, $f^{\prime} \in \mathcal{D}(\mu, f)$ if and only if there exists a distribution $g$ over $\mathcal{X}$ such that the following two conditions hold:

$$
\begin{aligned}
g\left(x^{\prime}\right) & =\sum_{x} g(x) \mathbf{P}\left(x^{\prime} \mid x, \mu(x), f\right), \text { for all } x^{\prime} ; \\
f^{\prime}(x, s) & =g(x) \cdot \mu(x)(s), \text { for all } x, s .
\end{aligned}
$$

Note that here $\mu(x)(s)$ is the probability assigned to pure action $s$ by the randomized strategy $\mu$ at state $x$. The first equation requires that $g$ is an invariant distribution of the state Markov process induced by $\mu$ and $f$ (recall Definition 9 of the transition kernel with mixed actions). The second equation requires $f^{\prime}$ to be derived from $g$ in the natural way, via $\mu$. As before, we let $\Phi(f)=\mathcal{D}(\mathcal{P}(f), f)$.

It is now straightforward to show that if Assumption 1 holds, then the result of Proposition 4 holds, i.e., $\Phi$ has a closed graph. Further, if Assumptions 1 and 4 hold, then the result of Proposition 9 holds as well. From this and the result in Proposition 5 we conclude that under those assumptions, a stationary equilibrium exists, and all SE are light-tailed (i.e., have finite 1- $p$ norm). The arguments involved are analogous to the existing proofs, and we omit the details.

We conclude by commenting on the restriction that the action space must be finite. From a computational standpoint this is not very restrictive, since in many applications discretization is required or can be used efficiently. From a theoretical standpoint, we can analyze games with general compact Euclidean action spaces using techniques similar to this paper, at the expense of additional measure-theoretic complexity, since now the population state-action profile is a measure over a continuous extended state space.

\section{B Approximation: Sequence of Payoff Functions}

In many models of interest it is more reasonable to assume that the payoff function explicitly depends on the number of agents. To study these environments, in this section we consider a sequence of payoff functions indexed by the number of agents, $\pi_{m}(x, a, f)$. Here, the profit function $\pi$ is a limit: $\lim _{m \rightarrow \infty} \pi_{m}(x, a, f)=$ $\pi(x, a, f)$. See Section 4 for concrete examples.

In this case, the actual expected net present value of a player using a cognizant strategy $\mu^{\prime}$ when every other of the $m-1$ players uses an oblivious strategy $\mu$ is given by equation (5), but where $\pi$ is replaced by $\pi_{m}$. That is, if the number of players is $m$, the payoff obtained each period is given by $\pi_{m}$. Hence, with some abuse of notation, for this section, we define:

$$
\begin{aligned}
V^{(m)}\left(x, f \mid \mu^{\prime}, \boldsymbol{\mu}^{(m-1)}\right) & \triangleq \\
& \mathbb{E}\left[\sum_{t=0}^{\infty} \beta^{t} \pi_{m}\left(x_{i, t}, a_{i, t}, \boldsymbol{f}_{-i, t}^{(m)}\right) \mid x_{i, 0}=x, f_{-i, 0}^{(m)}=f ; \mu_{i}=\mu^{\prime}, \boldsymbol{\mu}_{-i}=\boldsymbol{\mu}^{(m-1)}\right] .
\end{aligned}
$$

We generalize Theorem 2 for this setting. First, we need to strengthen Assumption 1.

Assumption 5. For each $m \in \mathbb{Z}_{+}$, Assumption 1 holds, with the following strengthened properties. 
1. Equicontinuity. The set of functions $\left\{\pi_{m}(x, a, f): m \in \mathbb{Z}_{+}\right\}$is jointly equicontinuous ${ }^{9}$ in $a \in \mathcal{A}$ and $f \in \mathfrak{F}_{p}$.

2. Uniform growth rate bound. There exist constants $K$ and $n \in \mathbb{Z}_{+}$such that $\sup _{m \in \mathbb{Z}_{+}, a \in \mathcal{A}, f \in \mathfrak{F}_{p}}\left|\pi_{m}(x, a, f)\right| \leq$ $K\left(1+\|x\|_{\infty}\right)^{n}$ for every $x \in \mathcal{X}$.

The following result is more general than Theorem 2, because the payoff function in the AME property depends on $m$.

Theorem 3 (AME). Suppose Assumption 5 holds. Let $(\mu, f)$ be a stationary equilibrium with $f \in \mathfrak{F}_{p}$. Then the AME property holds for $(\mu, f)$.

The proof is similar to Theorem 2, but requires an additional step to accommodate the sequence of payoff functions. However, note that similar to Theorem 2 , the stationary equilibrium $(\mu, f)$ is fixed and is computed with the limit payoff function $\pi$. Alternatively, it is possible to define an "oblivious equilibrium" (OE) for each finite model. An OE is similar to SE in the sense that agents optimize assuming that the long run population state is constant; the main difference is that it is defined in a finite model rather than in the limit model. Under a uniform light-tail condition, it can be shown that the sequence of OE satisfies the AME property Weintraub et al. (2008). In addition, we conjecture that a version of the assumptions that guarantee existence of SE in Section 5, but that applies uniformly over all finite models, would guarantee that such a uniform light-tail condition holds. For clarity of presentation, we chose to work with the SE of the limit model directly.

Moreover, we believe that the existence result for the limit model that we provide is important, because even though OE might exist under mild conditions for each finite model, SE in the limit model may fail to exist. In particular, as we discuss in Section 4, this might be the case in applications that exhibit "increasing returns to scale". See in particular Sections 4.1, 4.2, and 4.3, for examples of how limit models are derived in specific applications, and also conditions in such models that ensure stationary equilibria provide accurate approximations.

\section{Additional Examples}

In this section we present two additional applications to our results, to a model of supply chain competition, and a model of consumer learning.

\section{C.1 Supply Chain Competition}

We now consider an example of supply chain competition among firms (Cachon and Lariviere 1999), where the firms use a common resource that is sold by a single supplier. The firms only interact with each other in the sourcing stage as the goods produced are assumed to be sold in independent markets.

\footnotetext{
${ }^{9}$ Let $\mathcal{X}$ and $\mathcal{Y}$ be two metric spaces, with metrics $d \mathcal{X}$ and $d \mathcal{Y}$ respectively. A set of functions $\mathcal{F}$ mapping $\mathcal{X}$ to $\mathcal{Y}$ is said to be equicontinuous at $x_{0} \in \mathcal{X}$, if for every $\epsilon>0$, there exists a $\delta>0$ such that $d_{\mathcal{Y}}\left(f(x), f\left(x_{0}\right)\right)<\epsilon$ for all $f \in \mathcal{F}$ and all $x$ such that $d_{\mathcal{X}}\left(x_{0}, x\right)<\delta$.
} 
States. We let the state $x_{i, t}$ be the inventory of goods held by firm $i$ at time $t$.

Actions. At each time period, the supplier runs an auction to sell the goods. Each firm $i$ places a bid $a_{i, t}$ at time $t$; for example, $a_{i, t}$ may denote the willingness-to-pay of the supplier, or it may be a two-dimensional bid consisting of desired payment and quantity. Since the interaction between firms is via their action profiles we again assume that the action taken by a firm lies in a finite subset $S$ of the integer lattice.

Transition dynamics. Suppose that each firm $i$ sees demand $d_{i, t}$ at time $t$; we assume $d_{i, t}$ are i.i.d. and independent across firms, with bounded nonnegative support and positive expected value. Further, suppose that when a firm bids $s$ and the population state-action profile is $f$, the firm receives an allocation $\xi(s, f)$. Then the state evolution for a firm $i$ is given by $x_{i, t+1}=\max \left\{x_{i, t}-d_{i, t}, 0\right\}+\xi\left(s_{i, t}, \boldsymbol{f}_{-i, t}^{(m)}\right)$. Note that $\xi$ depends on $\boldsymbol{f}_{-i, t}^{(m)}$ only through the marginal distribution over actions. We make the natural assumptions that $\xi(s, f)$ is increasing in $s$ and decreasing in $f$ (where the set of distributions is ordered in the first order stochastic dominance sense). Thus the transition kernel captures inventory evolution in the usual way: demand consumes inventory, and procurement restocks inventory. The amount of resource procured by a firm and the price it pays depends on its own bid, as well as bids of other firms competing for the resource.

As one example of how $\xi$ might arise, suppose that the supplier uses a proportional allocation mechanism (Kelly 1997). In such a mechanism, the bid $s$ denotes the total amount a firm pays. Further, suppose the total quantity $Q_{m}$ of the resource available scales with the number of firms, i.e., $Q_{m}=m Q$. Let $k(s \mid f)=\sum_{x} f(x, s)$ denote the fraction of agents bidding $s$ in population state-action profile $f$.

As $m \rightarrow \infty$, and introducing $R$ as a small "reserve" bid that ensures the denominator is always nonzero, we obtain the following limiting proportional allocation function: $\xi(s, f)=s Q /\left(R+\sum_{s^{\prime}} s^{\prime} k\left(s^{\prime} \mid f\right)\right)$. Note that this expression is increasing in $s$ and decreasing in $f$.

Payoffs. A firm earns revenue for demand served, and incurs a cost both for holding inventory, as well as for procuring additional goods via restocking. We assume every firm faces an exogenous retail price $\phi$. (Heterogeneity in the retail price could be captured via the description in Section 3.5.) Let $h$ be the unit cost of holding inventory for one period and let $\Omega(s, f)$ be the procurement payment made by a firm with bid $s$, when the population state-action profile is $f$; of course, $\Omega$ also depends on $f$ only through $k(\cdot \mid f)$. In general we assume that $\Omega$ is increasing in $f$ for each fixed $s$. In the proportional allocation mechanism described above, we simply have $\Omega(s, f)=s$. Since the demand is i.i.d., the single period payoff for a firm is given by the expected payoff it receives, where the expectation is over the demand uncertainty; i.e. $\pi(x, s, f)=\phi \mathbb{E}[\min \{d, x\}]-h x-\Omega(s, f)$.

Discussion. We have the following proposition.

Proposition 10. Suppose that d has positive expected value. Then there exists an SE for the supply chain competition model with the proportional allocation mechanism, and all SE possess the AME property.

Proof. We present the proof in a more general setting, and specialize to the proportional allocation mechanism. If $\xi$ and $\Omega$ are uniformly bounded and appropriately continuous in $f$ for each pure action $s$, then Assumption 1 follows in a straightforward manner. For example, in the proportional allocation mechanism with a positive reserve bid $R$, note that $\xi$ is continuous in $f$ in the 1- $p$ norm with $p=1$, since $\xi$ depends on $f$ through its first moment. Since this is a model with finite action spaces, the result of Proposition 5 
also applies. Thus, as before, the proof is reduced to determining whether Assumption 4 holds for the given model.

As before, Assumption 4.2, Assumption 4.3, and Assumption 4.4 are easy to check. Assumption 4.1 follows because the payoff function is decreasing in $x$ for large $x$. Finally, suppose $0 \in S$ and $\xi(0, f)=0$ for all $f$; this will be the case, for example, in the proportional allocation mechanism with reserve $R$. Then if $\mathcal{A}^{\prime}=\{0\}$, it follows that Assumption 4.5 holds, as long as (1) $d$ has positive expected value; and (2) bidding zero is myopically optimal, and this induces negative drift in the inventory level. Note that bidding zero is myopically optimal for the proportional allocation mechanism, and this induces negative drift in the inventory. Using Corollary 1 and Theorem 2 the result follows.

More generally, for other choices of allocation mechanism, it can be shown that the same result holds if $d$ has positive expected value and the following conditions hold: (1) if $\xi$ and $\Omega$ are uniformly bounded and appropriately continuous in $f$ for each pure action $s$; (2) $0 \in S$ and $\xi(0, f)=0$ for all $f$; and (3) bidding zero maximizes a firm's single period payoff, and this induces negative drift in the inventory level.

In this model, decreasing returns to higher states are naturally enforced because the payoff function becomes decreasing in the state as the state grows. Simply because holding inventory is costly, firms prefer not to become arbitrarily large. Thus in this model light tails in the population state can be guaranteed under fairly weak assumptions on the model primitives.

\section{C.2 Consumer Learning}

In this section, we analyze a model of social learning. Imagine a scenario where a group of individuals decide to consume a product (e.g., visiting a restaurant). These individuals learn from each other's experience, perhaps through product reviews or word-of-mouth (see, for example, Ching 2010).

States. We let $x_{i, t}$ be the experience level of an individual at time $t$.

Actions. At each time period $t$, an individual invests an "effort" $a_{i, t} \in[0, \bar{a}]$ in searching for a new product.

Payoffs. At each time period, an individual selects a product to consume. The quality of the product is a normally distributed random variable $Q$ with a distribution given by $Q \sim \mathcal{N}(\gamma a, \omega(x, f))$, where $\gamma>0$ is a constant. Thus, the average quality of the product is proportional to the amount of effort made. Furthermore, the variance of the product is dependent on both individual and population experience levels.

We assume that $\omega(x, f)$ is continuous in the population state $f$ (in an appropriate norm, cf. Section 5). We make the natural assumption that $\omega(x, f)$ is a nonincreasing function of $f$ and strictly decreasing in $x$ (where the set of distributions is ordered in the first order stochastic dominance sense). This is natural as we expect that as an individual's experience increases or if she can learn from highly expert people, the randomness in choosing a product will decrease. We also assume that there exists constants $\sigma_{L}, \sigma_{H}$, such that $\sigma_{L}^{2} \leq \omega(x, f) \leq \sigma_{H}^{2}$.

The individual receives a utility $U(Q)$, where $U(\cdot)$ is a nondecreasing concave function of the quality. For concreteness, we let $U(Q)=1-e^{-Q}$. Since at each time, the individual selects the product or the restaurant in an i.i.d. manner, the single period payoff is given by $\pi(x, a, f)=E[U(Q) \mid Q \sim \mathcal{N}(\gamma a, \omega(x, f))]-$ $d a=1-e^{-\gamma a+\frac{1}{2} \omega(x, f)}-d a$, where $d$ is the marginal cost of effort. 
Transition dynamics. An individual's experience level is improved as she expends effort because she learns more about the quality of products. However, this experience level also depreciates over time; this depreciation is assumed to be player-specific and comes about because an individual's tastes may change over time. Thus, an individual's experience evolves (independently of the experience of others or their investments) in a stochastic manner. Several specifications for the transition kernel satisfying our assumptions can be used; for concreteness we assume that the dynamics are the same as those described in Section 4.1.

Discussion. Our main result is the following proposition.

Proposition 11. Suppose that:

$$
d \geq \gamma e^{-\gamma c_{0}+\frac{1}{2} \sigma_{H}^{2}}
$$

where $c_{0}=\delta /(\alpha(1-\delta))$. Then there exists an SE for the consumer learning model, and all SE possess the AME property.

Proof. Note that $\omega(x, f)<\sigma_{H}^{2}$ and thus the growth rate bound in Assumption 1 is trivially satisfied. If $\omega(x, f)$ is continuous in $f$ (in the appropriate 1-p norm), then Assumption 1 follows in a straightforward manner. To verify that $\Phi(f)$ is convex, we note that Assumption 3 will hold if $\pi(x, a, f)$ is strictly increasing in $x$ and concave in $a$. Since $\omega(x, f)$ is strictly decreasing in $x$, these conditions are naturally satisfied for our model. Thus, to complete the proof, we need to verify Assumption 4.

It is straightforward to check that Assumption 4.2-4 hold; we omit the details. Assumption 4.1 follows since $\omega(x, f)$ is nonincreasing in $x$ and bounded below, so $\omega(x, f)-\omega(x+\Delta, f) \rightarrow 0$ as $x \rightarrow \infty$. In order for Assumption 4.5 to hold, we require $\mathcal{A}^{\prime}$ to contain all myopically optimal actions. A straightforward calculation shows that $\arg \max _{a} \pi(x, a, f)=a^{*}(x, f)$, where

$$
a^{*}(x, f)=\frac{1}{2 \gamma} \omega(x, f)-\frac{1}{\gamma} \log \left(\frac{d}{\gamma}\right)
$$

for simplicity we assume $0<a^{*}(x, f)<\bar{a}$ for all $x, f$, though an analogous argument holds otherwise. Thus we define $\mathcal{A}^{\prime}=\left[0, a_{\max }\right]$, where:

$$
a_{\max }=\frac{1}{2 \gamma} \sigma_{H}^{2}-\frac{1}{\gamma} \log \left(\frac{d}{\gamma}\right) .
$$

To verify Assumption 4.5(a), note that if $a \notin \mathcal{A}^{\prime}$, then:

$$
\begin{aligned}
\pi\left(x, a^{*}(x, f), f\right)-\pi(x, a, f) & =e^{-\gamma a^{*}(x, f)+\frac{1}{2} \omega(x, f)}\left(e^{-\gamma\left(a-a^{*}(x, f)\right)}-1\right)+d\left(a-a^{*}(x, f)\right) \\
& =\frac{d}{\gamma} \kappa\left(a-a^{*}(x, f)\right),
\end{aligned}
$$

where $\kappa(x)=e^{-\gamma x}-1+\gamma x$, which is strictly increasing and nonnegative with $\kappa(0)=0$. Here the preceding derivation follows by observing that the optimality condition for $a^{*}(x, f)$ ensures that $\gamma e^{-\gamma a^{*}(x, f)+\frac{1}{2} \omega(x, f)}=$ d. Thus Assumption 4.5(a) holds.

When do the actions in $\mathcal{A}^{\prime}$ produce negative drift in the state? For the dynamics given in Section 4.1, one can easily verify that the drift is negative if the action is sufficiently small; in particular, the drift is negative 
for any action $a$ such that:

$$
a<\frac{\delta}{(1-\delta) \alpha} \triangleq c_{0}
$$

where $\delta \in(0,1)$ is the probability that the experience depreciates and $\alpha>0$ controls the probability that a player is successful in improving the experience. The above inequality is satisfied by all $a^{\prime} \in \mathcal{A}^{\prime}$ if :

$$
d \geq \gamma e^{-\gamma c_{0}+\frac{1}{2} \sigma_{H}^{2}}
$$

Using Corollary 1 and Theorem 2 the result follows.

Recall that $\delta \in(0,1)$ is the probability that the experience depreciates and $\alpha>0$ controls the probability that a player is successful in improving the experience. The right hand side is an upper bound to the marginal gain in utility due to effort, at effort level $c_{0}$; while the left hand side is the marginal cost of effort. Thus the condition (14) can be interpreted as a requirement that the marginal cost of effort should be sufficiently large relative to the marginal gain in utility due to effort. Otherwise, an individual's effort level when her experience is high will cause her state to continue to increase, so a light-tailed SE may not exist. Hence we see the same dichotomy as before: decreasing returns to higher states yield existence of SE and the AME property, while increasing returns may not.

\section{Existence and AME: Preliminary Lemmas}

We begin with the following lemma, which follows from the growth rate bound and bounded increments in Assumption 1.

Lemma 1. Suppose Assumption 1 holds. Let $x_{0}=x$. Let $a_{t} \in \mathcal{A}$ be any sequence of (possibly history dependent) actions, and let $f_{t} \in \mathfrak{F}$ be any sequence of (possibly history dependent) population states. Let $x_{t}$ be the state sequence generated, i.e., $x_{t} \sim \mathbf{P}\left(\cdot \mid x_{t-1}, a_{t-1}, f_{t-1}\right)$. Then for all $T \geq 0$, there exists $C(x, T)<\infty$ such that $\mathbb{E}\left[\sum_{t=T}^{\infty} \beta^{t}\left|\pi\left(x_{t}, a_{t}, f_{t}\right)\right| \mid x_{0}=x\right] \leq C(x, T)$. Further, $C(x, T) \rightarrow 0$ as $T \rightarrow \infty$.

Proof. Observe that by Assumption 1, the increments are bounded. Thus starting from state $x$, we have $\left\|x_{t}\right\|_{\infty} \leq\|x\|_{\infty}+t M$. Again by Assumption $1,\left|\pi\left(x_{t}, a_{t}, f_{t}\right)\right| \leq K\left(1+\left\|x_{t}\right\|_{\infty}\right)^{n}$. Therefore:

$$
\mathbb{E}\left[\sum_{t=T}^{\infty} \beta^{t}\left|\pi\left(x_{t}, a_{t}, f_{t}\right)\right| \mid x_{0}=x\right] \leq K \sum_{t=T}^{\infty} \beta^{t}\left(1+\|x\|_{\infty}+t M\right)^{n} .
$$

We define $C(x, 0)$ as the right hand side above when $T=0$ :

$$
C(x, 0)=K \sum_{t=0}^{\infty} \beta^{t}\left(1+\|x\|_{\infty}+t M\right)^{n}
$$

Observe that $C(x, 0)<\infty$. 
We now reason as follows for $T \geq 1$ :

$$
\begin{aligned}
K \sum_{t=T}^{\infty} \beta^{t}\left(1+\|x\|_{\infty}+t M\right)^{n} & =K \beta^{T} \sum_{t=0}^{\infty} \beta^{t}\left(1+\|x\|_{\infty}+t M+T M\right)^{n} \\
& =K \beta^{T} \sum_{t=0}^{\infty} \beta^{t} \sum_{j=0}^{n}\left(\begin{array}{c}
n \\
j
\end{array}\right)\left(1+\|x\|_{\infty}+t M\right)^{j}(T M)^{n-j} \\
& \leq K \beta^{T} \sum_{t=0}^{\infty} \beta^{t} \sum_{j=0}^{n}\left(\begin{array}{c}
n \\
j
\end{array}\right)\left(1+\|x\|_{\infty}+t M\right)^{n}(T M)^{n} \\
& =K \beta^{T} 2^{n}(T M)^{n} \sum_{t=0}^{\infty} \beta^{t}\left(1+\|x\|_{\infty}+t M\right)^{n} \\
& =C(x, 0) \beta^{T}(2 M T)^{n}
\end{aligned}
$$

Here the inequality holds because $1+\|x\|_{\infty}+t M \geq 1, M \geq 0$, and $T \geq 1$. So for $T \geq 1$, define:

$$
C(x, T)=C(x, 0) \beta^{T}(2 M T)^{n}
$$

Then $C(x, T) \rightarrow 0$ as $T \rightarrow \infty$, as required.

We now show that the Bellman equation holds for the dynamic program solved by a single agent given a population state $f$. Given our unbounded state space, our proof involves the use of a weighted sup norm, defined as follows. For each $x \in \mathcal{X}$, let $W(x)=\left(1+\|x\|_{\infty}\right)^{n}$. For a function $F: \mathcal{X} \rightarrow \mathbb{R}$, define:

$$
\|F\|_{W-\infty}=\sup _{x \in \mathcal{X}}\left|\frac{F(x)}{W(x)}\right|
$$

This is the weighted sup norm with weight function $W$. We let $B(\mathcal{X})$ denote the set of all functions $F$ : $\mathcal{X} \rightarrow \mathbb{R}$ such that $\|F\|_{W-\infty}<\infty$.

Let $T_{f}$ denote the dynamic programming operator with population state $f:$ given a function $F: \mathcal{X} \rightarrow \mathbb{R}$, we have $\left(T_{f} F\right)(x)=\sup _{a \in \mathcal{A}}\left\{\pi(x, a, f)+\beta \sum_{x^{\prime} \in \mathcal{X}} F\left(x^{\prime}\right) \mathbf{P}\left(x^{\prime} \mid x, a, f\right)\right\}$. We define $T_{f}^{k}$ to be the composition of the mapping $T_{f}$ with itself $k$ times. The following lemma applies standard dynamic programming arguments.

Lemma 2. Suppose Assumption 1 holds. For all $f \in \mathfrak{F}$, if $F \in B(\mathcal{X})$ then $T_{f} F \in B(\mathcal{X})$. Further, there exist $k, \rho$ independent of $f$ with $0<\rho<1$ such that $T_{f}$ is a $k$-stage $\rho$-contraction on $B(\mathcal{X})$; i.e., if $F, F^{\prime} \in B(\mathcal{X})$, then for all $f:\left\|T_{f}^{k} F-T_{f}^{k} F^{\prime}\right\|_{W-\infty} \leq \rho\left\|F-F^{\prime}\right\|_{W-\infty}$.

In particular, value iteration converges to $\tilde{V}^{*}(\cdot \mid f) \in B(\mathcal{X})$ from any initial value function in $B(\mathcal{X})$, and for all $f \in \mathfrak{F}$ and $x \in \mathcal{X}$, the Bellman equation holds:

$$
\tilde{V}^{*}(x \mid f)=\sup _{a \in \mathcal{A}}\left\{\pi(x, a, f)+\beta \sum_{x^{\prime} \in \mathcal{X}} \tilde{V}^{*}\left(x^{\prime} \mid f\right) \mathbf{P}\left(x^{\prime} \mid x, a, f\right)\right\} .
$$

Further, $\tilde{V}^{*}(x \mid f)$ is continuous in $f \in \mathfrak{F}_{p}$. 
Finally, there exists at least one optimal oblivious strategy among all (possibly history-dependent, possibly randomized) strategies; i.e., $\mathcal{P}(f)$ is nonempty for all $f \in \mathfrak{F}$. An oblivious strategy $\mu \in \mathfrak{M}_{O}$ is optimal given $f$ if and only if $\mu(x)$ achieves the maximum on the right hand side of (16) for every $x \in \mathcal{X}$.

Proof. We have the following three properties:

1. By growth rate bound in Assumption 1 we have $\sup _{a}|\pi(x, a, f)| / W(x) \leq K$ for all $x$.

2. We have:

$$
\bar{W}(x)=\sup _{a \in \mathcal{A}} \sum_{x^{\prime}} \mathbf{P}\left(x^{\prime} \mid x, a, f\right) W\left(x^{\prime}\right) \leq\left(1+\|x\|_{\infty}+M\right)^{n},
$$

since the increments are bounded (Assumption 1). Thus $\bar{W}(x) / W(x) \leq(1+M)^{n}$ for all $x$.

3. Finally, fix $\rho$ such that $0<\rho<1$ and let:

$$
\bar{W}_{k}(x)=\sup _{\mu \in \mathfrak{M}_{O}} \mathbf{E}\left[W\left(x_{k}\right) \mid x_{0}=x, \mu\right]
$$

where the state evolves according to $x_{t+1} \sim \mathbf{P}\left(\cdot \mid x_{t}, \mu\left(x_{t}\right), f\right)$. By bounded increments in Assumption 1, we have:

$$
\beta^{k} \bar{W}_{k}(x) \leq \beta^{k}\left(1+\|x\|_{\infty}+k M\right)^{n} \leq \beta^{k}(1+k M)^{n} W(x) .
$$

By choosing $k$ sufficiently large so that $\beta^{k}(1+k M)^{n}<\rho$, we have:

$$
\beta^{k} \bar{W}_{k}(x) \leq \rho W(x)
$$

Given (1)-(3), by standard arguments (see, e.g., Bertsekas 2007), it follows that $T_{f}$ is a $k$-stage $\rho$ contraction with respect to the weighted sup norm, value iteration converges to $\tilde{V}^{*}(\cdot \mid f)$, the Bellman equation holds, and any (stationary, nonrandomized) oblivious strategy that maximizes the right hand side in (16) for each $x \in \mathcal{X}$ is optimal. Observe that since $\tilde{V}^{*}(\cdot \mid f) \in B(\mathcal{X})$ for any $f$, it follows that $\tilde{V}^{*}(x \mid f)<\infty$ for all $x$. In fact, by Lemma $1,\left|\tilde{V}^{*}(x \mid f)\right| \leq C(x, 0)$ for all $x$.

Next we show that $\tilde{V}^{*}(x \mid f)$ is continuous in $f$. Define $Z(x)=0$ for all $x$, and let $V_{f}^{(\ell)}=T_{f}^{\ell} Z$. We first show that $V_{f}^{(\ell)}(x)$ is continuous in $f$. To see this, we proceed by induction. The result is trivially true at $\ell=0$. Next, observe that $\pi(x, a, f)$ is jointly continuous in $a$ and $f$ for each fixed $x$ by Assumption 1 . Suppose $V_{f}^{(\ell)}(x)$ is continuous in $f$ for each $x$; then $V_{f}^{(\ell)}\left(x^{\prime}\right) \mathbf{P}\left(x^{\prime} \mid x, a, f\right)$ is jointly continuous in $a$ and $f$ for each fixed $x, x^{\prime}$. Since the kernel has bounded increments from Assumption 1, we conclude that $\sum_{x^{\prime}} V_{f}^{(\ell)}\left(x^{\prime}\right) \mathbf{P}\left(x^{\prime} \mid x, a, f\right)$ is jointly continuous in $a$ and $f$ for each fixed $x$. It follows by Berge's maximum theorem (Aliprantis and Border 2006) that $V_{f}^{(\ell+1)}(x)$ is continuous in $f$.

Fix $\epsilon>0$. Since $T_{f}$ is a $k$-stage $\rho$-contraction in the weighted sup norm for every $f$, it follows that for all sufficiently large $\ell$, for every $f$ there holds:

$$
\left|V_{f}^{(\ell)}(x)-\tilde{V}^{*}(x \mid f)\right| \leq W(x) \epsilon
$$


So now suppose that $f_{n} \rightarrow f$ in the 1-p norm. Since $V_{f}^{\ell}(x)$ is continuous in $f$, for all sufficiently large $n$ there holds:

$$
\left|V_{f_{n}}^{(\ell)}(x)-V_{f}^{(\ell)}(x)\right| \leq \epsilon .
$$

Thus using the triangle inequality, for all sufficiently large $n$ we have:

$$
\left|\tilde{V}^{*}(x \mid f)-\tilde{V}^{*}\left(x \mid f_{n}\right)\right| \leq(2 W(x)+1) \epsilon .
$$

Since $\epsilon$ was arbitrary it follows that the left hand side approaches zero as $n \rightarrow \infty$, as required. Finally, observe that by a similar argument as above,

$$
\sum_{x^{\prime}} \tilde{V}^{*}\left(x^{\prime} \mid f\right) \mathbf{P}\left(x^{\prime} \mid x, a, f\right)
$$

is a continuous function of $a$ for each fixed $x$ and $f$; since $\pi(x, a, f)$ is also continuous in $a$ for each fixed $f$, the right hand side of (16) is continuous in $a$ for each fixed $f$. Since $\mathcal{A}$ is compact, it follows that there exists an optimal action at each state $x$, and thus there exists an optimal strategy given $f$.

\section{E Existence: Proof}

\section{E.1 Closed Graph: Proof}

Throughout this subsection we suppose Assumption 1 holds.

Lemma 3. For each $f, \mathcal{P}(f)$ is compact; further, the correspondence $\mathcal{P}$ is upper hemicontinuous on $\mathfrak{F}_{p}$.

Proof. By Assumption $1, \pi(x, a, f)$ is jointly continuous in $a$ and $f$. Lemma 2 establishes that the optimal oblivious value function $\tilde{V}^{*}(x \mid f)$ is continuous in $f$, and so as in the proof of that lemma, it follows that for a fixed state $x, \pi(x, a, f)+\beta \sum_{x^{\prime}} \tilde{V}^{*}\left(x^{\prime} \mid f\right) \mathbf{P}\left(x^{\prime} \mid x, a, f\right)$ is finite and jointly continuous in $a$ and $f$. Define the set $\mathcal{P}_{x}(f) \subset \mathcal{A}$ as the set of actions that achieve the maximum on the right hand side of (16); this is nonempty as $\mathcal{A}$ is compact (Assumption 1) and the right hand side is continuous in $a$. By Berge's maximum theorem, for each $x$ the correspondence $\mathcal{P}_{x}$ is upper hemicontinuous with compact values (Aliprantis and Border 2006).

By Lemma 2, $\mu \in \mathcal{P}(f)$ if and only if $\mu(x) \in \mathcal{P}_{x}(f)$ for each $x$. Note that we have endowed the set of strategies with the topology of pointwise convergence. The range space of $\mathcal{P}$ is an infinite product of the compact action space $\mathcal{A}$ (Assumption 1) over the countable state space. Hence by Tychonoff's theorem (Aliprantis and Border 2006), the range space of $\mathcal{P}$ is compact. Further, since $\mathcal{P}_{x}$ is compact-valued, it follows that $\mathcal{P}$ is compact-valued. Since $\mathcal{P}_{x}(f)$ is compact-valued and upper hemicontinuous, the Closed Graph Theorem ensures that $\mathcal{P}_{x}$ has a closed graph (Aliprantis and Border 2006). This in turn ensures that $\mathcal{P}$ has closed graph; again by the Closed Graph Theorem, we conclude that $\mathcal{P}$ is upper hemicontinuous.

Proof of Proposition 4. Suppose $f_{k} \rightarrow f$ in the 1-p norm, and that $g_{k} \rightarrow g$ in the 1-p norm, where $g_{k} \in \Phi\left(f_{k}\right)$ for all $k$. We must show that $g \in \Phi(f)$. For each $k$, let $\mu_{k} \in \mathcal{P}\left(f_{k}\right)$ be an optimal oblivious 
strategy such that $g_{k} \in \mathcal{D}\left(\mu_{k}, f_{k}\right)$. As in the proof of Lemma 3, the range space of $\mathcal{P}$ is compact in the topology of pointwise convergence; therefore, taking subsequences if necessary, we can assume without loss of generality that $\mu_{k}$ converges to some strategy $\mu \in \mathfrak{M}_{O}$ pointwise. By upper hemicontinuity of $\mathcal{P}$ (Lemma 3), we have $\mu \in \mathcal{P}(f)$.

By definition of $\mathcal{D}$, it follows that for all $x$ :

$$
g_{k}(x)=\sum_{x^{\prime}} g_{k}\left(x^{\prime}\right) \mathbf{P}\left(x \mid x^{\prime}, \mu_{k}\left(x^{\prime}\right), f_{k}\right)
$$

Since $\mathbf{P}\left(x \mid x^{\prime}, a, f\right)$ is jointly continuous in action and population state (Assumption 1), it follows that for all $x$ and $x^{\prime}$ :

$$
\mathbf{P}\left(x \mid x^{\prime}, \mu_{k}\left(x^{\prime}\right), f_{k}\right) \rightarrow \mathbf{P}\left(x \mid x^{\prime}, \mu\left(x^{\prime}\right), f\right)
$$

as $k \rightarrow \infty$. Further, if $g_{k} \rightarrow g$ in the 1-p norm, then in particular, $g_{k}(x) \rightarrow g(x)$ for all $x$. Finally, observe that for all $a$ and $f$, we have $\mathbf{P}\left(x \mid x^{\prime}, a, f\right)=0$ for all states $x^{\prime}$ such that $\left\|x^{\prime}-x\right\|_{\infty}>M$, since increments are bounded (Assumption 1). Thus:

$$
\sum_{x^{\prime}} g_{k}\left(x^{\prime}\right) \mathbf{P}\left(x \mid x^{\prime}, \mu_{k}\left(x^{\prime}\right), f_{k}\right) \rightarrow \sum_{x^{\prime}} g\left(x^{\prime}\right) \mathbf{P}\left(x \mid x^{\prime}, \mu\left(x^{\prime}\right), f\right)
$$

as $k \rightarrow \infty$. Taking the limit as $k \rightarrow \infty$ on both sides of (17) yields:

$$
g(x)=\sum_{x^{\prime}} g\left(x^{\prime}\right) \mathbf{P}\left(x \mid x^{\prime}, \mu\left(x^{\prime}\right), f\right)
$$

which establishes that $g \in \mathcal{D}(\mu, f)$. Since we had $\mu \in \mathcal{P}(f)$, we conclude $g \in \Phi(f)$, as required.

\section{E.2 Convexity: Proof}

Proof of Proposition 5. Fix $f \in \mathfrak{F}_{p}$, and let $g_{1}, g_{2}$ be elements of $\Phi(f)$. Let $\mu_{1}, \mu_{2} \in \mathcal{P}(f)$ be strategies such that $g_{i} \in \mathcal{D}\left(\mu_{i}, f\right), i=1,2$. Then for $i=1,2$ and all $x^{\prime} \in \mathcal{X}$, we have:

$$
g_{i}\left(x^{\prime}\right)=\sum_{x} g_{i}\left(x^{\prime}\right) \mathbf{P}\left(x^{\prime} \mid x, \mu_{i}(x), f\right)
$$

Fix $\delta, 0 \leq \delta \leq 1$, and for each $x$, define $g(x)$ by:

$$
g(x)=\delta g_{1}(x)+(1-\delta) g_{2}(x)
$$

We must show $g \in \Phi(f)$. Define a new strategy $\mu$ as follows: for each $x$ such that $g(x)>0$,

$$
\mu(x)=\frac{\delta g_{1}(x) \mu_{1}(x)+(1-\delta) g_{2}(x) \mu_{2}(x)}{g(x)} .
$$

For each $x$ such that $g(x)=0$, let $\mu(x)=\mu_{1}(x)$. 
We claim that $\mu \in \mathcal{P}(f)$, i.e., $\mu$ is an optimal oblivious strategy given $f$; and that $g \in \mathcal{D}(\mu, f)$, i.e., that $g$ is an invariant distribution given strategy $\mu$ and population state $f$. This suffices to establish that $g \in \Phi(f)$.

To establish the claim, first observe that under Definition 9, the right hand side of (16) is linear in $a$. Thus any convex combination of two optimal actions is also an optimal action. This establishes that for every $x, \mu(x)$ achieves the maximum on the right hand side of (16); so we conclude $\mu \in \mathcal{P}(f)$.

Let $T=\{x: g(x)>0\}$. Then:

$$
\begin{aligned}
g\left(x^{\prime}\right) & =\delta g_{1}\left(x^{\prime}\right)+(1-\delta) g_{2}\left(x^{\prime}\right) \\
& =\sum_{x} \delta g_{1}(x) \mathbf{P}\left(x^{\prime} \mid x, \mu_{1}(x), f\right)+(1-\delta) g_{2}(x) \mathbf{P}\left(x^{\prime} \mid x, \mu_{2}(x), f\right) \\
& =\sum_{x} \sum_{s}\left(\delta g_{1}(x) \mu_{1}(x)(s)+(1-\delta) g_{2}(x) \mu_{2}(x)(s)\right) \mathbf{P}\left(x^{\prime} \mid x, s, f\right) \\
& =\sum_{x \in T} \sum_{s} g(x) \mu(x)(s) \mathbf{P}\left(x^{\prime} \mid x, s, f\right) .
\end{aligned}
$$

The first equality is the definition of $g\left(x^{\prime}\right)$, and the second equality follows by expanding the invariant distribution equations for $g_{1}$ and $g_{2}$. The third equality follows by expanding the sum over pure actions $s$. Finally, in the last equality, we substitute the definition of $g(x)$, and we also observe that for $x \notin T$, $g(x)=0$ — and therefore, $g_{1}(x)=g_{2}(x)=0$. Since $g(x)=0$ for $x \notin T$, it follows that:

$$
\sum_{x \notin T} \sum_{s} g(x) \mu(x)(s) \mathbf{P}\left(x^{\prime} \mid x, s, f\right)=0 .
$$

It follows that:

$$
g\left(x^{\prime}\right)=\sum_{x} g(x) \mathbf{P}\left(x^{\prime} \mid x, \mu(x), f\right)
$$

as required.

Lemma 4. Suppose Assumptions 1 and 3 hold. Then $\tilde{V}^{*}(\cdot \mid f)$ is strictly increasing for every $f \in \mathfrak{F}_{p}$, and the right hand side of (16) is strictly concave in a.

Proof. Define $Z(x)=0$ for all $x$, and let $V_{f}^{(\ell)}=T_{f}^{\ell} Z$. Observe that if $V_{f}^{(\ell)}$ is nondecreasing, then under the conditions of the lemma, it follows that $V_{f}^{(\ell+1)}$ will be nondecreasing. Taking the limit as $n \rightarrow \infty$, we conclude (from convergence of value iteration) that $\tilde{V}^{*}(\cdot \mid f)$ is nondecreasing, and thus the right hand side of (16) is strictly increasing in $x$. From this it follows that in fact, $\tilde{V}^{*}(\cdot \mid f)$ is strictly increasing.

Since $\tilde{V}^{*}(\cdot \mid f)$ is strictly increasing, $\pi(x, a, f)$ is concave in $a$, and the kernel is stochastically concave in $a$, with at least one of the last two strictly concave, it follows that the right hand side of (16) is strictly concave in $a$.

Proof of Proposition 7. Under Assumptions 1 and 2, the optimal action in (16) can be shown to be unique (see Doraszelski and Satterthwaite 2010). It follows that $\mathcal{P}(f)$ is a singleton.

From the preceding lemma, Assumptions 1 and 3 together also guarantee a unique optimal solution in the right hand side of (16), for every $x \in \mathcal{X}$. Thus under either of these conditions the optimal strategy given 
$f$ is unique, i.e., $\mathcal{P}(f)$ is a singleton. The result follows by Proposition 6.

\section{E.3 Compactness: Proof}

Throughout this subsection we suppose $\mathcal{X}=\mathbb{Z}_{+}^{d}$ and that Assumptions 1 and 4 are in effect.

Lemma 5. Given $x^{\prime} \geq x, x, x^{\prime} \in \mathcal{X}, a \in \mathcal{A}$, and $f \in \mathfrak{F}$, there exists a probability space with random variables $\xi^{\prime} \sim \mathbf{Q}\left(\cdot \mid x^{\prime}, a, f\right), \xi \sim \mathbf{Q}(\cdot \mid x, a, f)$, such that $\xi^{\prime} \leq \xi$ almost surely, and $x^{\prime}+\xi^{\prime} \geq x+\xi$ almost surely.

Proof. The proof uses a coupling argument. Let $U$ be a uniform random variable on $[0,1]$. Let $F_{\ell}\left(\right.$ resp., $\left.F_{\ell}^{\prime}\right)$ be the cumulative distribution function of $\mathbf{Q}_{\ell}(\cdot \mid x, a, f)$ (resp., $\mathbf{Q}_{\ell}\left(\cdot \mid x^{\prime}, a, f\right)$ ), and let $G_{\ell}$ (resp., $\left.G_{\ell}^{\prime}\right)$ be the cumulative distribution function of $\mathbf{P}_{\ell}(\cdot \mid x, a, f)$ (resp., $\mathbf{P}_{\ell}\left(\cdot \mid x^{\prime}, a, f\right)$ ). By Assumption $4, \mathbf{P}_{\ell}(\cdot \mid x, a, f)$ is stochastically nondecreasing in $x$, and $\mathbf{Q}_{\ell}(\cdot \mid x, a, f)$ is stochastically nonincreasing in $x$. Thus for all $z$, $F_{\ell}(z) \leq F_{\ell}^{\prime}(z)$, but for all $y, G_{\ell}(y) \geq G_{\ell}^{\prime}(y)$; further, $G_{\ell}(y)=F_{\ell}\left(y-x_{\ell}\right)$ (and $G_{\ell}^{\prime}(y)=F_{\ell}^{\prime}\left(y-x_{\ell}^{\prime}\right)$ ). Let $\xi_{\ell}=\inf \left\{z_{\ell}: F_{\ell}\left(z_{\ell}\right) \geq U\right\}$, and let $\xi_{\ell}^{\prime}=\inf \left\{z_{\ell}: F^{\prime}\left(z_{\ell}\right) \geq U\right\}$. Then $\xi_{\ell} \geq \xi_{\ell}^{\prime}$ for all $\ell$, i.e., $\xi \geq \xi^{\prime}$. Rewriting the definitions, we also have $x_{\ell}+\xi_{\ell}=\inf \left\{y_{\ell}: F_{\ell}\left(y_{\ell}-x_{\ell}\right) \geq U\right\}$, and $x_{\ell}^{\prime}+\xi_{\ell}^{\prime}=\inf \left\{y_{\ell}: F_{\ell}^{\prime}\left(y_{\ell}-x_{\ell}^{\prime}\right) \geq U\right\}$, i.e., $x_{\ell}+\xi_{\ell}=\inf \left\{y_{\ell}: G_{\ell}\left(y_{\ell}\right) \geq U\right\}$, and $x_{\ell}^{\prime}+\xi_{\ell}^{\prime}=\inf \left\{y_{\ell}: G_{\ell}^{\prime}\left(y_{\ell}\right) \geq U\right\}$. Thus $x_{\ell}+\xi_{\ell} \leq x_{\ell}^{\prime}+\xi_{\ell}^{\prime}$ for all $\ell$, i.e., $x^{\prime}+\xi^{\prime} \geq x+\xi$, as required.

Given a set $S$ define $\rho_{\infty}(x, S)=\inf _{y \in S}\|x-y\|_{\infty}$. Thus $\rho_{\infty}$ gives the $\infty$-norm distance to a set. We have the following lemma.

Lemma 6. As $\|x\|_{\infty} \rightarrow \infty, \sup _{f \in \mathfrak{F}} \sup _{\mu \in \mathcal{P}(f)} \rho_{\infty}\left(\mu(x), \mathcal{A}^{\prime}\right) \rightarrow 0$.

Proof. Suppose the statement of the lemma fails; then there exists $r>0$ and a sequence $f_{n} \in \mathfrak{F}, \mu_{n} \in$ $\mathcal{P}\left(f_{n}\right)$, and $x_{n}$ (where $\left.\left\|x_{n}\right\|_{\infty} \rightarrow \infty\right)$ such that $\rho_{\infty}\left(\mu_{n}\left(x_{n}\right), \mathcal{A}^{\prime}\right) \geq r$ for all $n$. We use this fact to construct a profitable deviation from the policy $\mu_{n}$, for sufficiently large $n$.

Observe that by Assumption 4, there must exist $a_{n}^{\prime} \in \mathcal{A}^{\prime}$ with $a_{n}^{\prime} \leq \mu_{n}\left(x_{n}\right)$, such that:

$$
\pi\left(x_{n}, a_{n}^{\prime}, f_{n}\right)-\pi\left(x_{n}, \mu_{n}\left(x_{n}\right), f_{n}\right) \geq \kappa\left(\left\|a_{n}^{\prime}-\mu_{n}\left(x_{n}\right)\right\|_{\infty}\right) \geq \kappa(r)>0,
$$

where the last inequality follows since $\kappa$ is strictly increasing with $\kappa(0)=0$. Importantly, note the bound on the right hand side is a constant, independent of $n$.

Let $x_{0, n}=x_{n}$, and let $x_{t, n}$ and $a_{t, n}$ denote the state and action sequence realized under $\mu_{n}$, starting from $x_{0, n}$, under the kernel $\mathbf{P}\left(\cdot \mid x, a, f_{n}\right)$. We consider a deviation from $\mu_{n}$, where at time 0 , instead of playing $a_{0, n}=\mu_{n}\left(x_{n}\right)$, the agent plays $a_{0, n}^{\prime}=a_{n}^{\prime}$; and then at all times in the future, the agent follows the same actions as the original sequence, i.e., $a_{t, n}^{\prime}=a_{t, n}$. Let $x_{t, n}^{\prime}$ denote the resulting state sequence.

Since the kernel is stochastically nondecreasing in $a$, and $a_{n}^{\prime} \leq a_{n}$, it follows that there exists a common probability space together with increments $\xi_{0, n}, \xi_{0, n}^{\prime}$, such that $\xi_{0, n} \sim \mathbf{Q}\left(\cdot \mid x_{n}, a_{n}, f_{n}\right), \xi_{0, n}^{\prime} \sim$ $\mathbf{Q}\left(\cdot \mid x_{n}, a_{n}^{\prime}, f_{n}\right)$, and $\xi_{0, n}^{\prime} \leq \xi_{0, n}$ almost surely. Thus we can couple together $x_{1, n}$ and $x_{1, n}^{\prime}$, by letting $x_{1, n}=x_{n}+\xi_{0, n}$, and $x_{1, n}^{\prime}=x_{n}+\xi_{0, n}^{\prime}$. In particular, observe that with these definitions we have $x_{1, n} \geq x_{1, n}^{\prime}$. Let $\Delta_{n}=\xi_{0, n}-\xi_{0, n}^{\prime} \geq 0$. Note that $\left\|\Delta_{n}\right\|_{\infty} \leq 2 M$, by Assumption 1 (bounded increments). 
Next, it follows from Lemma 5 that there exists a probability space with random variables $\xi_{1, n}, \xi_{1, n}^{\prime}$ such that $\xi_{1, n} \sim \mathbf{Q}\left(\cdot \mid x_{1, n}, a_{1, n}, f_{n}\right)$ and $\xi_{1, n}^{\prime} \sim \mathbf{Q}\left(\cdot \mid x_{1, n}^{\prime}, a_{1, n}, f_{n}\right), \xi_{1, n} \leq \xi_{1, n}^{\prime}$ almost surely, and yet $x_{1, n}+\xi_{1, n} \geq x_{1, n}^{\prime}+\xi_{1, n}^{\prime}$ almost surely. Thus we can couple together $x_{2, n}$ and $x_{2, n}^{\prime}$, by letting $x_{2, n}=$ $x_{1, n}+\xi_{1, n}$, and let $x_{2, n}^{\prime}=x_{1, n}^{\prime}+\xi_{1, n}^{\prime}$. Proceeding inductively, it can be shown that there exists a joint probability measure under which $0 \leq x_{t, n}-x_{t, n}^{\prime} \leq \Delta_{n}$, almost surely, for all $t \geq 1$ (where the inequalities are interpreted coordinatewise); this follows by a standard application of the Kolmogorov extension theorem.

We now compare the payoffs obtained under these two sequences. We have:

$$
\begin{aligned}
\mathbb{E}\left[\sum_{t} \beta^{t}\left(\pi\left(x_{t, n}, a_{t, n}, f_{n}\right)-\pi\left(x_{t, n}^{\prime}, a_{t, n}^{\prime}, f_{n}\right)\right)\right]=\pi\left(x_{n}, \mu_{n}\left(x_{n}\right), f_{n}\right)-\pi\left(x_{n}, a_{n}^{\prime}, f_{n}\right) \\
+\mathbb{E}\left[\sum_{t \geq 1} \beta^{t}\left(\pi\left(x_{t, n}, a_{t, n}, f_{n}\right)-\pi\left(x_{t, n}^{\prime}, a_{t, n}, f_{n}\right)\right)\right] \\
\leq-\kappa(r)+\mathbb{E}\left[\sum_{t \geq 1} \beta^{t} \sup _{\delta \geq 0:\|\delta\|_{\infty} \leq 2 M} \sup _{a, f}\left(\pi\left(x_{t, n}, a, f\right)-\pi\left(x_{t, n}-\delta, a, f\right)\right)\right] .
\end{aligned}
$$

Since increments are bounded (Assumption 4), in time $t$, the maximum distance the state could have moved in each coordinate from the initial state $x$ is bounded by $t M$. Thus if $x_{0, n}=x_{n}$, then:

$\sup _{\delta \geq 0:\|\delta\|_{\infty} \leq 2 M} \sup _{a, f}\left(\pi\left(x_{t, n}, a, f\right)-\pi\left(x_{t, n}-\delta, a, f\right)\right) \leq \sup _{\substack{\delta \geq 0, \epsilon:\|\delta\|_{\infty} \leq 2 M,\|\epsilon\|_{\infty} \leq t M}} \sup _{a, f}\left(\pi\left(x_{n}+\epsilon, a, f\right)-\pi\left(x_{n}+\epsilon-\delta, a, f\right)\right)$.

Let $A_{t, n}$ denote the right hand side of the preceding equation; note that this is a deterministic quantity, and that the supremum is over a finite set. Thus from Assumption 4, we have $\lim _{\sup } \rightarrow \infty A_{t, n} \leq 0$.

Finally, observe that since $\lim \sup _{\|x\|_{\infty} \rightarrow \infty} \sup _{a, f}(\pi(x+\delta, a, f)-\pi(x, a, f)) \leq 0$, it follows that:

$$
\sup _{y \in \mathbb{Z}_{+}^{d}, \delta \geq 0:\|\delta\|_{\infty} \leq 2 M} \sup _{a, f}(\pi(y, a, f)-\pi(y-\delta, a, f))<\infty .
$$

We denote the left hand side of the preceding inequality by $D$. Note that this is a constant independent of $n$.

Combining our arguments, we have that for all sufficiently large $n$, there holds:

$$
\mathbb{E}\left[\sum_{t} \beta^{t}\left(\pi\left(x_{t, n}, a_{t, n}, f_{n}\right)-\pi\left(x_{t, n}^{\prime}, a_{t, n}^{\prime}, f_{n}\right)\right)\right] \leq-\kappa(r)+\sum_{t=1}^{T} \beta^{t} A_{t, n}+\frac{\beta^{T} D}{1-\beta} .
$$

By taking $T$ sufficiently large, we can ensure that the last term on the right hand side is strictly less than $\kappa(r) / 2$; and by then taking $n$ sufficiently large, we can ensure that the second term on the right hand side is also strictly less than $\kappa(r) / 2$. Thus for sufficiently large $n$, we conclude that the left hand side is negativecontradicting optimality of $\mu_{n}$. The lemma follows.

Lemma 7. There exists $\bar{\epsilon}>0$ and $\bar{K}$ such that for all $\ell$ and all $x$ with $x_{\ell} \geq \bar{K}$, $\sup _{f} \sup _{\mu \in \mathcal{P}(f)} \sum_{z_{\ell}} z_{\ell} \mathbf{Q}_{\ell}\left(z_{\ell} \mid x, \mu(x), f\right)<-\bar{\epsilon}$. 
Proof. Fix $\epsilon>0$ so that for all $\ell$ and all $x^{\prime}$ with $x_{\ell}^{\prime} \geq K^{\prime}, \sup _{a^{\prime} \in \mathcal{A}^{\prime}} \sup _{f} \sum_{z_{\ell}} z_{\ell} \mathbf{Q}_{\ell}\left(z_{\ell} \mid x^{\prime}, a^{\prime}, f\right)<-\epsilon$; such a constant exists by the last part of Assumption 4. Observe that since $\mathcal{A}$ is compact and $\sup _{f} \sum_{z_{\ell}} z_{\ell} \mathbf{Q}_{\ell}\left(z_{\ell} \mid x, a, f\right)$ is continuous in $a$ (Assumption 4), it follows that $\sup _{f} \sum_{z_{\ell}} z_{\ell} \mathbf{Q}_{\ell}\left(z_{\ell} \mid x, a, f\right)$ is in fact uniformly continuous in $a \in \mathcal{A}$. Let $e^{(\ell)}$ denote the $\ell^{\prime}$ th standard basis vector (i.e., $e_{\ell^{\prime}}^{(\ell)}=0$ for $\ell^{\prime} \neq \ell$, and $e_{\ell}^{(\ell)}=1$ ). By uniform continuity, we can conclude there must exist a $\delta_{\ell}>0$ such that if $\left\|a-a^{\prime}\right\|_{\infty}<\delta_{\ell}$, then:

$$
\left|\sup _{f} \sum_{z_{\ell}} z_{\ell} \mathbf{Q}_{\ell}\left(z_{\ell} \mid K^{\prime} e^{(\ell)}, a, f\right)-\sup _{f} \sum_{z_{\ell}} z_{\ell} \mathbf{Q}_{\ell}\left(z_{\ell} \mid K^{\prime} e^{\ell}, a^{\prime}, f\right)\right|<\epsilon / 2 .
$$

Note in particular, if $\rho_{\infty}\left(a, \mathcal{A}^{\prime}\right)<\delta_{\ell}$, then there exists $a^{\prime} \in \mathcal{A}^{\prime}$ with $\left\|a-a^{\prime}\right\|_{\infty}<\delta_{\ell}$. By our choice of $\epsilon$ we have $\sup _{f} \sum_{z_{\ell}} z_{\ell} \mathbf{Q}_{\ell}\left(z_{\ell} \mid K^{\prime} e^{(\ell)}, a, f\right)<-\frac{\epsilon}{2}$. Now let $\delta=\min \left\{\delta_{1}, \ldots, \delta_{d}\right\}$. Since the increment kernel is stochastically nonincreasing in $x$, it follows that if $\rho_{\infty}\left(a, \mathcal{A}^{\prime}\right)<\delta$ and $x_{\ell} \geq K^{\prime}$, then $\sup _{f} \sum_{z_{\ell}} z_{\ell} \mathbf{Q}_{\ell}\left(z_{\ell} \mid x, a, f\right)<-\frac{\epsilon}{2}$. Since $\sup _{f} \sup _{\mu \in \mathcal{P}(f)} \rho_{\infty}\left(\mu(x), \mathcal{A}^{\prime}\right) \rightarrow 0$ as $\|x\|_{\infty} \rightarrow \infty$, the result follows if we let $\bar{\epsilon}=\epsilon / 2$.

Lemma 8. For every $f \in \mathfrak{F}, \Phi(f)$ is nonempty.

Proof. As described in the discussion of Section 5.3, it suffices to show that the state Markov chain induced by an optimal oblivious strategy possesses at least one invariant distribution-i.e., that $\mathcal{D}(\mu, f)$ is nonempty, where $\mu$ is an optimal oblivious strategy given $f$.

We first show that for every $f$ and every $\mu \in \mathcal{P}(f)$, the Markov chain on $\mathcal{X}$ induced by $\mu$ and $f$ has at least one closed class. Let $S=\left\{x:\|x\|_{\infty} \leq \bar{K}+M\right\}$. By Lemma 7, if $x \notin S$, then there exists some state $x^{\prime}$ with $\mathbf{P}\left(x^{\prime} \mid x, \mu(x), f\right)>0$ such that $x_{\ell}^{\prime} \leq x_{\ell}-\bar{\epsilon}$ for all $\ell$ where $x_{\ell}>\bar{K}$. On the other hand, since increments are bounded, for any $\ell$ where $x_{\ell} \leq \bar{K}$, we have $x_{\ell}^{\prime} \leq \bar{K}+M$. Applying this fact inductively, we find that for any $x \notin S$, there must exist a positive probability sequence of states from $x$ to $S$; i.e., a sequence $y_{0}, y_{1}, y_{2}, \ldots, y_{\tau}$ such that $y_{0}=x, y_{\tau} \in S$, and $\mathbf{P}\left(y_{t} \mid y_{t-1}, \mu\left(y_{t-1}\right), f\right)>0$ for all $t$. We say that $S$ is reachable from $x$.

So now suppose the chain induced by $\mu$ and $f$ has no closed class. Fix $x_{0} \in S$. Since the class containing $x_{0}$ is not closed, there must exist a state $x^{\prime}$ reachable from $x_{0}$ with positive probability, such that the chain never returns to $x_{0}$ starting from $x^{\prime}$. If $x^{\prime} \in S$, let $x_{1}=x^{\prime}$. If $x^{\prime} \notin S$, then using the argument in the preceding paragraph, there must exist a state $x_{1} \in S$ reachable from $x^{\prime}$. Arguing inductively, we can construct a sequence of states $x_{0}, x_{1}, x_{2}, \ldots$ where $x_{t} \in S$ for all $t$, and yet $x_{0}, \ldots, x_{t-1}$ are not reachable from $x_{t}$. But $S$ is finite, so at least one state must repeat in this sequence-contradicting the construction. We conclude that the chain must have at least one closed class.

To complete the proof, we use a Foster-Lyapunov argument. Let $U(x)=\sum_{\ell} x_{\ell}^{2}$. Then $\{x \in \mathcal{X}$ : $U(x) \leq R\}$ is finite for all $R$. So now let $\omega=\left(2 d \bar{K} M+d M^{2}+1\right) /(2 \bar{\epsilon})$, and suppose $\|x\|_{\infty}>\max \{\omega, \bar{K}\}$. 
We reason as follows:

$$
\begin{aligned}
\sum_{x^{\prime}} U\left(x^{\prime}\right) \mathbf{P}\left(x^{\prime} \mid x, \mu(x), f\right) & =U(x)+2 \sum_{\ell} x_{\ell} \sum_{z_{\ell}} z_{\ell} \mathbf{Q}_{\ell}\left(z_{\ell} \mid x, \mu(x), f\right)+\sum_{\ell} \sum_{z_{\ell}} z_{\ell}^{2} \mathbf{Q}_{\ell}\left(z_{\ell} \mid x, \mu(x), f\right) \\
& \leq U(x)+2 \sum_{\ell: x_{\ell} \leq \bar{K}} M x_{\ell}-2 \sum_{\ell: x_{\ell}>\bar{K}} \bar{\epsilon} x_{\ell}+d M^{2} \leq U(x)-1 .
\end{aligned}
$$

The first equality follows by definition of $\mathbf{Q}$ and $U$, and multiplicative separability of $\mathbf{Q}$. The next step follows since increments are bounded (Assumption 4), and by applying Lemma 7 for $x_{\ell}>\bar{K}$. The last inequality follows from the fact that the state space is $d$-dimensional, $\|x\|_{\infty}>\max \{\bar{K}, \omega\}$, and by definition of $\omega$. Since increments are bounded, it is trivial that for every $R$ :

$$
\sup _{x:\|x\|_{\infty} \leq R}\left(\sum_{x^{\prime}} U\left(x^{\prime}\right) \mathbf{P}\left(x^{\prime} \mid x, \mu(x), f\right)-U(x)\right)<\infty .
$$

It follows by the Foster-Lyapunov criterion that every closed class of the Markov chain induced by $\mu$ is positive recurrent, as required (Hajek 1982, Meyn and Tweedie 1993, Glynn and Zeevi 2006).

Lemma 9. For every $\eta \in \mathbb{Z}_{+}$, $\sup _{f} \sup _{\phi \in \Phi(f)} \sum_{x}\|x\|_{\eta}^{\eta} \phi(x)<\infty$.

Proof. We again use a Foster-Lyapunov argument. We proceed by induction; the claim is clearly true if $\eta=0$. So assume the claim is true up to $\eta-1$; in particular, define:

$$
\alpha_{k}=\sup _{f} \sup _{\phi \in \Phi(f)} \sum_{x}\|x\|_{k}^{k} \phi(x)
$$

for $k=0, \ldots, \eta-1$. Fix $f$, and let $\mu \in \mathcal{P}(f)$ be an optimal oblivious strategy given $f$. The preceding lemma establishes that the Markov chain induced by $\mu$ possesses at least one invariant distribution. Let $U(x)=\sum_{\ell} x_{\ell}^{\eta+1}$. Then we have:

$$
\begin{aligned}
\sum_{x^{\prime}} U\left(x^{\prime}\right) \mathbf{P}\left(x^{\prime} \mid x, \mu(x), f\right)= & \sum_{\ell} \sum_{z_{\ell}}\left(x_{\ell}+z_{\ell}\right)^{\eta+1} \mathbf{Q}_{\ell}\left(z_{\ell} \mid x, \mu(x), f\right) \\
= & \sum_{\ell} \sum_{z_{\ell}} \sum_{k=0}^{\eta+1}\left(\begin{array}{c}
\eta+1 \\
k
\end{array}\right) x_{\ell}^{k} z_{\ell}^{\eta+1-k} \mathbf{Q}_{\ell}\left(z_{\ell} \mid x, \mu(x), f\right) \\
= & U(x)+(\eta+1) \sum_{\ell} x_{\ell}^{\eta} \sum_{z_{\ell}} z_{\ell} \mathbf{Q}_{\ell}\left(z_{\ell} \mid x, \mu(x), f\right) \\
& +\sum_{\ell} \sum_{z_{\ell}} \sum_{k=0}^{\eta-1}\left(\begin{array}{c}
\eta+1 \\
k
\end{array}\right) x_{\ell}^{k} z_{\ell}^{\eta+1-k} \mathbf{Q}(z \mid x, \mu(x), f) .
\end{aligned}
$$

Define $g(x)$ as:

$$
g(x)=\sum_{k=0}^{\eta-1}\left(\begin{array}{c}
\eta+1 \\
k
\end{array}\right) M^{\eta+1-k} \sum_{\ell} x_{\ell}^{k}
$$


By the inductive hypothesis,

$$
\gamma \triangleq \sup _{f} \sup _{\phi \in \Phi(f)} \sum_{x} g(x) \phi(x)<\infty .
$$

Further, by Lemma 7, for all $\ell$ and all $x$ such that $x_{\ell} \geq \bar{K}$, we have:

$$
\sum_{z_{\ell}} z_{\ell} \mathbf{Q}_{\ell}\left(z_{\ell} \mid x, \mu(x), f\right)<-\bar{\epsilon}
$$

Define $h(x)$ as:

$$
h(x)=-(\eta+1) M \sum_{\ell: x_{\ell} \leq \bar{K}} x_{\ell}^{\eta}+\bar{\epsilon}(\eta+1) \sum_{\ell: x_{\ell}>\bar{K}} x_{\ell}^{\eta} .
$$

It follows that:

$$
\sum_{x^{\prime}} U\left(x^{\prime}\right) \mathbf{P}\left(x^{\prime} \mid x, \mu(x), f\right)-U(x) \leq-h(x)+g(x) .
$$

Now fix any distribution $\phi \in \mathcal{D}(\mu, f)$. Since the Markov chain induced by $\mu$ and $f$ must be irreducible on the support of $\phi$, it follows by the Foster-Lyapunov criterion (Meyn and Tweedie 1993) that:

$$
\sum_{x} h(x) \phi(x) \leq \sum_{x} g(x) \phi(x) \leq \gamma
$$

Rearranging terms, we conclude that:

$$
\sum_{x}\left(\sum_{\ell: x_{\ell}>\bar{K}} x_{\ell}^{\eta}\right) \phi(x) \leq \frac{\gamma}{\bar{\epsilon}(\eta+1)}+\frac{d M \bar{K}^{\eta}}{\bar{\epsilon}} .
$$

Thus:

$$
\sum_{x}\|x\|_{\eta}^{\eta} \phi(x) \leq \frac{\gamma}{\bar{\epsilon}(\eta+1)}+\left(\frac{d M}{\bar{\epsilon}}+d\right) \bar{K}^{\eta}
$$

(Recall that the sum is only over $x \in \mathbb{Z}_{+}^{d}$.) Since the right hand side is finite and independent of $f$ and $\phi$, the result follows.

Proof of Proposition 9. We have already established that $\Phi(f)$ is nonempty for all $f \in \mathfrak{F}$ in Lemma 8 . Define $B=\sup _{f} \sup _{\phi \in \Phi(f)} \sum_{x}\|x\|_{p+1}^{p+1} \phi(x)<\infty$, where the inequality is the result of Lemma 9 .

We define the set $\mathfrak{C}=\left\{f \in \mathfrak{F}: \sum_{x}\|x\|_{p+1}^{p+1} f(x) \leq B\right\}$. By the preceding observation, $\Phi(\mathfrak{F}) \subset \mathfrak{C}$. It is clear that $\mathfrak{C}$ is nonempty and convex. It remains to be shown that $\mathfrak{C}$ is compact in the 1-p-norm. It is straightforward to check that $\mathfrak{C}$ is complete; we show that $\mathfrak{C}$ is totally bounded, thus establishing compactness.

Fix $\epsilon>0$. Choose $K_{\epsilon}$ so that $B / K_{\epsilon}<\epsilon$. Then for all $f \in \mathfrak{C}$ :

$$
\sum_{x:\|x\|_{\infty} \geq K_{\epsilon}}\|x\|_{p}^{p} f(x) \leq \frac{B}{K_{\epsilon}}<\epsilon .
$$


Let $S_{\epsilon}=\left\{x:\|x\|_{\infty}<K_{\epsilon}\right\}$ and let $\mathfrak{S}_{C}$ be the projection of $\mathfrak{C}$ onto $S_{\epsilon}$; i.e.,

$$
\mathfrak{S}_{C}=\left\{g \in \mathbb{R}^{S_{\epsilon}}: \exists f \in \mathfrak{C} \text { with } g(x)=f(x) \forall x \in S_{\epsilon}\right\}
$$

It is straightforward to check that $\mathfrak{S}_{C}$ is a compact subset of the finite-dimensional space $\mathbb{R}^{S_{\epsilon}}$; so let $f_{1}, \ldots, f_{k} \in \mathfrak{S}_{C}$ be a $\epsilon$-cover of $\mathfrak{S}_{C}$ (i.e., $\mathfrak{S}_{C}$ is covered by the balls around $f_{1}, \ldots, f_{k}$ of radius $\epsilon$ in the 1 - $p$-norm). Then it follows that $f_{1}, \ldots, f_{k}$ is a $2 \epsilon$-cover of $\mathfrak{C}$, since (19) bounds the tail of any $f \in \mathfrak{C}$ by $\epsilon$. This establishes that $\mathfrak{C}$ is totally bounded in the 1 - $p$-norm, as required.

\section{E.4 Finite Actions}

We conclude by briefly discussing how the proof of Proposition 9 may be adapted in the case of finite action spaces (cf. Definition 9). Suppose that $S \subset \mathbb{R}^{q}$ is a finite set. We now show that as long as Assumption 4 holds with respect to pure actions - i.e., with $\mathcal{A}$ replaced by $S$-Proposition 9 continues to hold.

Lemma 5 follows as before, except with $\mathcal{A}$ replaced by $S$. Lemma 6 follows the same argument if we restrict attention to pure strategies $\mu$, i.e., strategies that take a pure action in every state. Let $\hat{\mathcal{P}}(f)$ denote the set of optimal pure strategies given $f$. Then Lemma 6 then yields that as $\|x\|_{\infty} \rightarrow \infty$ :

$$
\sup _{f} \sup _{\mu \in \hat{\mathcal{P}}(f)} \rho_{\infty}\left(\mu(x), \mathcal{A}^{\prime}\right) \rightarrow 0
$$

Since $\mathcal{A}^{\prime} \subset S$, it is finite as well. It follows that there exists $\zeta$ such that for $x$ such that $\|x\|_{\infty} \geq \zeta$, for all $f$, and all $\mu \in \hat{\mathcal{P}}(f)$, we have $\mu(x) \in \mathcal{A}^{\prime}$. From this and Assumption 4 the result of Lemma 7 holds for $\mu \in \hat{\mathcal{P}}(f)$, i.e., there exists $\epsilon>0$ such that for all $\ell$ and all $x$ with $x_{\ell} \geq K^{\prime}$,

$$
\sup _{f} \sup _{\mu \in \hat{\mathcal{P}}(f)} \sum_{z_{\ell}} z_{\ell} \mathbf{Q}_{\ell}\left(z_{\ell} \mid x, \mu(x), f\right)<-\epsilon
$$

To complete our proof, we need only note that the set of all optimal oblivious strategies $\mathcal{P}(f)$ can be obtained by pointwise convex combinations of optimal pure oblivious strategies; this follows from Bellman's equation and the fact that the payoff is linear in the mixed action. Thus we also have:

$$
\sup _{f} \sup _{\mu \in \mathcal{P}(f)} \sum_{z_{\ell}} z_{\ell} \mathbf{Q}_{\ell}\left(z_{\ell} \mid x, \mu(x), f\right)<-\epsilon .
$$

The remainder of the proof follows as before.

\section{F AME: Proof}

Throughout this section we suppose Assumption 1 holds. We begin by defining the following sets. 
Definition 11. For every $x \in \mathcal{X}$, define

$$
\mathcal{X}_{x}=\left\{z \in \mathcal{X} \mid \mathbf{P}(x \mid z, a, f)>0 \text { for some } a \in \mathcal{A} \text { and for some } f \in \mathfrak{F}_{p}\right\} .
$$

Also define $\mathcal{X}_{x, t}$ as

$$
\mathcal{X}_{x, t}=\left\{z \in \mathcal{X} \mid\|z\|_{\infty} \leq\|x\|_{\infty}+t M\right\}
$$

Thus, $\mathcal{X}_{x}$ is the set of all initial states that can result in the final state as $x$. Since the increments are bounded (Assumption 1), for every $x \in \mathcal{X}$, the set $\mathcal{X}_{x}$ is finite. The set $\mathcal{X}_{x, t}$ is a superset of all possible states that can be reached at time $t$ starting from state $x$ (since the increments are uniformly bounded over action $a$ and distribution $f$ ); note that $\mathcal{X}_{x, t}$ is finite as well.

The following key lemma establishes that as the number of players grows large, the population empirical distribution in a game with finitely many players approaches the limiting SE population. The result is similar in spirit to related results on mean field limits of interacting particle systems, cf. Sznitman (1991); there the main insight is that, under appropriate conditions, the stochastic evolution of a finite-dimensional interacting particle system approaches the deterministic mean field limit over finite time horizons. Our model introduces two sources of complexity. First, agents' state transitions are coupled, so the population state Markov process is not simply the aggregation of independent agent state dynamics. Second, our state space is unbounded, so additional care is required to ensure the tail of the population state distribution is controlled in games with a large but finite number of players. This is where the light tail condition plays a key role. Our proof proceeds by induction over time periods.

Lemma 10. Let $(\mu, f)$ be a stationary equilibrium with $f \in \mathfrak{F}_{p}$. Consider an m-player game. Let $x_{i, 0}^{(m)}=$ $x_{0}$ and suppose the initial state of every player (other than player $i$ ) is independently sampled from the distribution $f$. That is, suppose $x_{j, 0}^{(m)} \sim f$ for all $j \neq i$; let $f^{(m)} \in \mathfrak{F}^{(m)}$ denote the initial population state. Let $a_{i, t}^{(m)}$ be any sequence of (possibly random, possibly history dependent) actions. Suppose players' states evolve as $x_{i, t+1}^{(m)} \sim \mathbf{P}\left(\cdot \mid x_{i, t}^{(m)}, a_{i, t}^{(m)}, f_{-i, t}^{(m)}\right)$ and for all $j \neq i$, as $x_{j, t+1}^{(m)} \sim \mathbf{P}\left(\cdot \mid x_{j, t}^{(m)}, \mu\left(x_{j, t}^{(m)}\right), f_{-j, t}^{(m)}\right)$. Then, for every initial state $x_{0}$, for all times $t,\left\|f_{-i, t}^{(m)}-f\right\|_{1-p} \rightarrow 0$ almost surely ${ }^{10}$ as $m \rightarrow \infty$.

Proof. Note that $f \in \mathfrak{F}_{p}$ and hence $\|f\|_{1-p}<\infty$. Thus, given any $\epsilon>0$, there exists a finite set $\mathcal{C}_{\epsilon, f}$ such that:

$$
\sum_{x \notin \mathcal{C}_{\epsilon, f}}\|x\|_{p}^{p} f(x)<\epsilon .
$$

At $t=0$, we have

$$
f_{-i, 0}^{(m)}(x)=\frac{1}{m-1} \sum_{j=1}^{m-1} \mathbf{1}_{\left\{X_{j, 0}=x\right\}},
$$

\footnotetext{
${ }^{10}$ Note that the convergence is almost surely in the randomness associated with the initial population state.
} 
where $X_{j, 0}$ are i.i.d random variables distributed according to the distribution $f$. Define:

$$
Y_{j}=\left\|X_{j, 0}\right\|_{p}^{p} \mathbf{1}_{\left\{X_{j, 0} \notin \mathcal{C}_{\epsilon, f}\right\}} \cdot
$$

Note that the $Y_{j}$ are i.i.d. random variables, with:

$$
\mathbf{E}\left[Y_{j}\right]=\sum_{x \notin \mathcal{C}_{\epsilon, f}}\|x\|_{p}^{p} f(x) .
$$

Further, observe that:

$$
\sum_{x \notin \mathcal{C}_{\epsilon, f}}\|x\|_{p}^{p} f_{-i, 0}^{(m)}(x)=\frac{1}{m-1} \sum_{j=1}^{m-1} Y_{j}
$$

Thus by the strong law of large numbers, almost surely as $m \rightarrow \infty$,

$$
\sum_{x \notin \mathcal{C}_{\epsilon, f}}\|x\|_{p}^{p} f_{-i, 0}^{(m)}(x) \rightarrow \sum_{x \notin \mathcal{C}_{\epsilon, f}}\|x\|_{p}^{p} f(x)<\epsilon .
$$

Now observe that:

$$
\left\|f_{-i, 0}^{(m)}(x)-f\right\|_{1-p} \leq \sum_{x \in \mathcal{C}_{\epsilon, f}}\|x\|_{p}^{p}\left|f_{-i, 0}^{(m)}(x)-f(x)\right|+\sum_{x \notin \mathcal{C}_{\epsilon, f}}\|x\|_{p}^{p} f_{-i, 0}^{(m)}(x)+\sum_{x \notin \mathcal{C}_{\epsilon, f}}\|x\|_{p}^{p} f(x) .
$$

Each of the second and third terms on the right hand side is almost surely less than $\epsilon$ for sufficiently large $m$. For the first term, observe that $\left|f_{-i, 0}^{(m)}(x)-f(x)\right| \rightarrow 0$ almost surely, again by the strong law of large numbers (since $f^{(m)}(x)$ is the sample average of $m-1$ Bernoulli random variables with parameter $f(x)$ ). Thus the first term approaches zero almost surely as $m \rightarrow \infty$ by the bounded convergence theorem. Since $\epsilon$ was arbitrary, this proves that $\left\|f_{-i, 0}^{(m)}-f\right\|_{1-p} \rightarrow 0$ almost surely as $m \rightarrow \infty$.

We now use an induction argument; let us assume that, $\left\|f_{-i, \tau}^{(m)}-f\right\|_{1-p} \rightarrow 0$ almost surely as $m \rightarrow \infty$ for all times $\tau \leq t$. From the definition of $f_{-i, t+1}^{(m)}$ we have:

$$
f_{-i, t+1}^{(m)}(y)=\frac{1}{m-1} \sum_{j \neq i} \mathbf{1}_{\left\{x_{j, t+1}^{(m)}=y\right\}}
$$

where $x_{j, t+1}^{(m)} \sim \mathbf{P}\left(\cdot \mid x_{j, t}^{(m)}, \mu\left(x_{j, t}^{(m)}\right), f_{-j, t}^{(m)}\right)$ for all $j \neq i$. Note that if two players have same initial state, then the population state from their viewpoint is identical. That is, if $x_{j, t}^{(m)}=x_{k, t}^{(m)}$, then $f_{-j, t}^{(m)}(y)=f_{-k, t}^{(m)}(y)$ for all $y \in \mathcal{X}$. We can thus redefine the population state from the viewpoint of a player at a particular state. Let $\hat{f}_{t}^{(x, m)}$ be the the population state at time $t$ from the viewpoint of a player at state $x$. Then, if $x_{j, t}^{(m)}=x_{k, t}^{(m)}=x$, then for all $y \in \mathcal{X}, f_{-j, t}^{(m)}(y)=f_{-k, t}^{(m)}(y)=\hat{f}_{t}^{(x, m)}(y)$. Without loss of generality, we assume $m>1$. Let $\eta_{-i, t}^{(m)}(x)$ be the total number of players (excluding player $i$ ) that have their state at time $t$ as $x$, i.e., $\eta_{-i, t}^{(m)}(x)=(m-1) \boldsymbol{f}_{-i, t}^{(m)}(x)$. Note that $\eta_{-i, t}^{(m)}(x)=0$ if and only $\boldsymbol{f}_{-i, t}^{(m)}(x)=0$. We can now write 
$f_{-i, t+1}^{(m)}(y)$ as:

$$
\begin{aligned}
f_{-i, t+1}^{(m)}(y) & =\frac{1}{m-1} \sum_{x \in \mathcal{X}} \sum_{j=1}^{\eta_{-i, t}^{(m)}(x)} \mathbf{1}_{\left\{Y_{j, x, t}^{(m)}=y\right\}} \\
& =\sum_{x \in \mathcal{X}} \boldsymbol{f}_{-i, t}^{(m)}(x)\left[\frac{1}{\eta_{-i, t}^{(m)}(x)} \sum_{j=1}^{\eta_{-i, t}^{(m)}(x)} \mathbf{1}_{\left\{Y_{j, x, t}^{(m)}=y\right\}}\right] \\
& =\sum_{x \in \mathcal{X}_{y}} \boldsymbol{f}_{-i, t}^{(m)}(x)\left[\frac{1}{\eta_{-i, t}^{(m)}(x)} \sum_{j=1}^{\eta_{-i, t}^{(m)}(x)} \mathbf{1}_{\left\{Y_{j, x, t}^{(m)}=y\right\}}\right]
\end{aligned}
$$

where the last equality follows from the Definition 11 . Here, $Y_{j, x, t}^{(m)}$ are random variables that are independently drawn according to the transition kernel $\mathbf{P}\left(\cdot \mid x, \mu(x), \hat{f}_{t}^{(x, m)}\right)$. Note that if $\eta_{-i, t}^{(m)}(x)=0$, we interpret the term inside the parentheses as zero.

Let us now look at $\hat{f}_{t}^{(x, m)}$. We have

$$
\hat{f}_{t}^{(x, m)}(z)=\boldsymbol{f}_{-i, t}^{(m)}(z)+\frac{1}{m-1} \mathbf{1}_{\left\{x_{i, t}^{(m)}=z\right\}}-\frac{1}{m-1} \mathbf{1}_{\{z=x\}} .
$$

Consider $\left\|\hat{f}_{t}^{(x, m)}-f\right\|_{1-p}$. We have:

$$
\begin{aligned}
\left\|\hat{f}_{t}^{(x, m)}-f\right\|_{1-p} & =\sum_{z \in \mathcal{X}}\|z\|_{p}^{p}\left|\hat{f}_{t}^{(x, m)}(z)-f(z)\right| \\
& =\sum_{z \in \mathcal{X}}\|z\|_{p}^{p}\left|\boldsymbol{f}_{-i, t}^{(m)}(z)+\frac{1}{m-1} \mathbf{1}_{\left\{x_{i, t}^{(m)}=z\right\}}-\frac{1}{m-1} \mathbf{1}_{\{z=x\}}-f(z)\right| \\
& \leq \sum_{z \in \mathcal{X}}\|z\|_{p}^{p}\left|\boldsymbol{f}_{-i, t}^{(m)}(z)-f(z)\right|+\frac{1}{m-1} \sum_{z \in \mathcal{X}}\|z\|_{p}^{p} \mathbf{1}_{\left\{x_{i, t}^{(m)}=z\right\}}+\frac{1}{m-1} \sum_{z \in \mathcal{X}}\|z\|_{p}^{p} \mathbf{1}_{\{z=x\}} \\
& =\left\|\boldsymbol{f}_{-i, t}^{(m)}-f\right\|_{1-p}+\frac{1}{m-1} \sum_{z \in \mathcal{X}}\|z\|_{p}^{p} \mathbf{1}_{\left\{x_{i, t}^{(m)}=z\right\}}+\frac{1}{m-1} \sum_{z \in \mathcal{X}}\|z\|_{p}^{p} \mathbf{1}_{\{z=x\}}
\end{aligned}
$$

From the induction hypothesis, we have $\left\|\boldsymbol{f}_{-i, t}^{(m)}-f\right\|_{1-p} \rightarrow 0$ almost surely as $m \rightarrow \infty$. Note that at time $t$, $x_{i, t}^{(m)} \in \mathcal{X}_{x_{0}, t}$ from equation (21), and $\mathcal{X}_{x_{0}, t}$ is finite. Thus,

$$
\sup _{m} \sum_{z \in \mathcal{X}}\|z\|_{p}^{p} \mathbf{1}_{\left\{x_{i, t}^{(m)}=z\right\}}<\infty, \text { almost surely. }
$$

This implies that for all states $x \in \mathcal{X},\left\|\hat{f}_{t}^{(x, m)}-f\right\|_{1-p} \rightarrow 0$ almost surely as $m \rightarrow \infty$. From Assumption 1 , we know that the transition kernel is continuous in the population state $f$ (where $\mathfrak{F}_{p}$ is endowed with the 1- $p$ 
norm). Thus for every $x \in \mathcal{X}$, we have almost surely:

$$
\mathbf{P}\left(\cdot \mid x, \mu(x), \hat{f}_{t}^{(x, m)}\right) \rightarrow \mathbf{P}(\cdot \mid x, \mu(x), f),
$$

as $m \rightarrow \infty$.

Next, we show that $f_{-i, t+1}^{(m)}(y) \rightarrow f(y)$ almost surely as $m \rightarrow \infty$, for all $y$. We leverage equation (23). Observe that the set of points $x \in \mathcal{X}$ where $\|x\|_{p} \leq 1$ is finite, since $\mathcal{X}$ is a subset of an integer lattice. From the induction hypothesis, as $\sum_{x \in \mathcal{X}}\|x\|_{p}^{p}\left|\boldsymbol{f}_{-i, t}^{(m)}(x)-f(x)\right| \rightarrow 0$ almost surely as $m \rightarrow \infty$, it follows that $f_{-i, t}^{(m)}(x) \rightarrow f(x)$ almost surely for all $x \in \mathcal{X}$ as $x \rightarrow \infty$.

Suppose that $x \in \mathcal{X}_{y}$ and $f(x)>0$. Since $\boldsymbol{f}_{-i, t}^{(m)}(x) \rightarrow f(x)$, it follows that $\eta_{-i, t}^{(m)} \rightarrow \infty$ as $m \rightarrow \infty$, almost surely. Note that $Y_{j, x, t}^{(m)}$ are random variables that are independently drawn according to the transition kernel $\mathbf{P}\left(\cdot \mid x, \mu(x), \hat{f}_{t}^{(x, m)}\right)$. From equation (24), and Lemma 11, we get that for every $x, y \in \mathcal{X}$, there holds

$$
\frac{1}{\eta_{-i, t}^{(m)}(x)} \sum_{j=1}^{\eta_{-i, t}^{(m)}(x)} \mathbf{1}_{\left\{Y_{j, x, t}^{(m)}=y\right\}} \rightarrow \mathbf{P}(y \mid x, \mu(x), f)
$$

almost surely as $m \rightarrow \infty$.

On the other hand, suppose $x \in \mathcal{X}_{y}$ and $f(x)=0$. Again, since $\boldsymbol{f}_{-i, t}^{(m)}(x) \rightarrow f(x)$ as $x \rightarrow \infty$, it follows that as $m \rightarrow \infty$, almost surely:

$$
\boldsymbol{f}_{-i, t}^{(m)}(x)\left[\frac{1}{\eta_{-i, t}^{(m)}(x)} \sum_{j=1}^{\eta_{-i, t}^{(m)}(x)} \mathbf{1}_{\left\{Y_{j, x, t}^{(m)}=y\right\}}\right] \rightarrow 0
$$

since the term in brackets is nonnegative and bounded. (Recall we interpret the term in brackets as zero if $\boldsymbol{f}_{-i, t}^{(m)}(x)=0$.)

We conclude that, almost surely, as $m \rightarrow \infty$ :

$$
f_{-i, t+1}^{(m)}(y)=\sum_{x \in \mathcal{X}_{y}} \boldsymbol{f}_{-i, t}^{(m)}(x)\left[\frac{1}{\eta_{-i, t}^{(m)}(x)} \sum_{j=1}^{\eta_{-i, t}^{(m)}(x)} \mathbf{1}_{\left\{Y_{j, x, t}^{(m)}=y\right\}}\right] \rightarrow \sum_{x \in \mathcal{X}_{y}} f(x) \mathbf{P}(y \mid x, \mu(x), f)=f(y) .
$$

To complete the proof, we need to show that $\left\|f_{-i, t+1}^{(m)}-f\right\|_{1-p} \rightarrow 0$ almost surely as $m \rightarrow \infty$. Since $f_{-i, t}^{(m)}(x) \rightarrow f(x)$ almost surely, for all $\epsilon>0$ we have:

$$
\sum_{x \in \mathcal{C}_{\epsilon, f}}\|x\|_{p}^{p} \boldsymbol{f}_{-i, t}^{(m)}(x) \rightarrow \sum_{x \in \mathcal{C}_{\epsilon, f}}\|x\|_{p}^{p} f(x)
$$


This together with the fact that $\left\|\boldsymbol{f}_{-i, t}^{(m)}-f\right\|_{1-p} \rightarrow 0$ implies that, almost surely:

$$
\limsup _{m \rightarrow \infty} \sum_{x \in \mathcal{C}_{\epsilon, f}}\|x\|_{p}^{p} \boldsymbol{f}_{-i, t}^{(m)}(x)<\epsilon
$$

Now at time $t+1$, we have

$$
\begin{aligned}
\sum_{x \notin \mathcal{C}_{\epsilon, f}}\|x\|_{p}^{p} f_{-i, t+1}^{(m)} & =\sum_{x \notin \mathcal{C}_{\epsilon, f}} \sum_{\ell=1}^{d}\left|x_{\ell}\right|^{p} f_{-i, t+1}^{(m)}(x) \\
& \leq \sum_{x \notin \mathcal{C}_{\epsilon, f}} \sum_{\ell=1}^{d}\left(\left|x_{\ell}\right|+M\right)^{p} f_{-i, t}^{(m)}(x),
\end{aligned}
$$

where the equality follows because $\mathcal{X}$ is a subset of the $d$-dimensional integer lattice. The last inequality follows from the fact that the increments are bounded (Assumption 1). Without loss of generality, assume that $\left|x_{\ell}\right| \geq 1$ and that $M \geq 1$. Then we have:

$$
\begin{aligned}
\left(\left|x_{\ell}\right|+M\right)^{p} & =\sum_{j=1}^{p}\left(\begin{array}{c}
p \\
j
\end{array}\right)\left|x_{\ell}\right|^{j} M^{p-j} \\
& \leq \sum_{j=1}^{p}\left(\begin{array}{c}
p \\
j
\end{array}\right)\left|x_{\ell}\right|^{p} M^{p} \\
& =2^{p} M^{p}\left|x_{\ell}\right|^{p}=K_{1}\left|x_{\ell}\right|^{p},
\end{aligned}
$$

where we let $K_{1}=(2 M)^{p}$. Substituting in equation (26), we have, almost surely,

$$
\begin{aligned}
\limsup _{m \rightarrow \infty} \sum_{x \notin \mathcal{C}_{\epsilon, f}}\|x\|_{p}^{p} f_{-i, t+1}^{(m)} & \leq \sum_{x \notin \mathcal{C}_{\epsilon, f}} \sum_{\ell=1}^{d} K_{1}\left|x_{\ell}\right|^{p} f_{-i, t}^{(m)}(x) \\
& =K_{1} \sum_{x \notin \mathcal{C}_{\epsilon, f}}\|x\|_{p}^{p} f_{-i, t}^{(m)}(x) \\
& <K_{1} \epsilon
\end{aligned}
$$

where the last inequality follows from equation (25). Now observe that:

$$
\left\|f_{-i, t+1}^{(m)}-f\right\|_{1-p} \leq \sum_{x \in \mathcal{C}_{\epsilon, f}}\|x\|_{p}^{p}\left|f_{-i, t+1}^{(m)}(x)-f(x)\right|+\sum_{x \notin \mathcal{C}_{\epsilon, f}}\|x\|_{p}^{p} f_{-i, t+1}^{(m)}(x)+\sum_{x \notin \mathcal{C}_{\epsilon, f}}\|x\|_{p}^{p} f(x) .
$$

In taking a limsup on the left hand side, the second term on the right hand side is almost surely less than $K_{1} \epsilon$. From the definition of $\mathcal{C}_{\epsilon, f}$ and equation (22), we get that the third term on the right hand side is also less than $\epsilon$. Finally, since for every $x\left|f_{-i, t+1}^{(m)}(x)-f(x)\right| \rightarrow 0$ almost surely as $m \rightarrow \infty$, and $C_{\epsilon, f}$ is finite, the first term in the above equation approaches zero almost surely as $m \rightarrow \infty$ by the Bounded Convergence Theorem. Since $\epsilon$ was arbitrary, this proves the induction step and hence the lemma. 
The preceding proof uses the following refinement of the strong law of large numbers.

Lemma 11. Suppose $0 \leq p_{k} \leq 1$ for all $k$, and that $p_{k} \rightarrow p$ as $k \rightarrow \infty$. For each $k$, let $Y_{1}^{(k)}, \ldots, Y_{k}^{(k)}$ be i.i.d. Bernoulli random variables with parameter $p_{k}$. Then almost surely:

$$
\lim _{k \rightarrow \infty} \frac{1}{k} \sum_{i=1}^{k} Y_{i}^{(k)}=p
$$

Proof. Let $\epsilon>0$. By Hoeffding's inequality, we have:

$$
\operatorname{Prob}\left(\left|\frac{1}{k} \sum_{i=1}^{k} Y_{i}^{(k)}-p_{k}\right|>\epsilon\right) \leq 2 e^{-k \epsilon_{k}^{2}},
$$

since $0 \leq Y_{i}^{(k)} \leq 1$ for all $i, k$. Let $\epsilon_{k}=1 / k$; then by the Borel-Cantelli lemma, the event on the left hand side in the preceding expression occurs for only finitely many $k$, almost surely. In other words, almost surely:

$$
\lim _{k \rightarrow \infty}\left[p_{k}-\frac{1}{k} \sum_{i=1}^{k} Y_{i}^{(k)}\right]=0
$$

The result follows.

Before we prove the AME property, we need some additional notation. Let $(\mu, f)$ be a stationary equilibrium. Consider again an $m$ player game and focus on player $i$. Let $x_{i, 0}^{(m)}=x_{0}$ and assume that player $i$ uses a cognizant strategy $\mu_{m}$. The initial state of every other player $j \neq i$ is independently drawn from the distribution $f$, that is, $x_{j, 0}^{(m)} \sim f$. Denote the initial distribution of all $m-1$ players (excluding player $i$ ) by $f^{(m)} \in \mathfrak{F}^{(m)}$. The state evolution of player $i$ is given by

$$
x_{i, t+1}^{(m)} \sim \mathbf{P}\left(\cdot \mid x_{i, t}^{(m)}, a_{i, t}^{(m)}, \boldsymbol{f}_{-i, t}^{(m)}\right),
$$

where $a_{i, t}^{(m)}=\mu_{m}\left(x_{i, t}^{(m)}, \boldsymbol{f}_{-i, t}^{(m)}\right)$ and $\boldsymbol{f}_{-i, t}^{(m)}$ is the actual population distribution. Here the superscript $m$ on the state variable represents the fact that we are considering an $m$ player stochastic game. Let every other player $j$ use the oblivious strategy $\mu$ and thus their state evolution is given by

$$
x_{j, t+1}^{(m)} \sim \mathbf{P}\left(\cdot \mid x_{j, t}^{(m)}, \mu\left(x_{j, t}^{(m)}\right), f_{-j, t}^{(m)}\right) .
$$

Define $V^{(m)}\left(x, f^{(m)} \mid \mu_{m}, \boldsymbol{\mu}^{(m-1)}\right)$ to be the actual value function of player $i$, with its initial state $x$, the initial distribution of the rest of the population as $f^{(m)} \in \mathfrak{F}^{(m)}$, when the player uses a cognizant strategy $\mu_{m}$ and every other player uses an oblivious strategy $\mu$. We have

$$
\begin{array}{r}
V^{(m)}\left(x, f^{(m)} \mid \mu_{m}, \boldsymbol{\mu}^{(m-1)}\right)=\mathbb{E}\left[\sum_{t=0}^{\infty} \beta^{t} \pi\left(x_{i, t}, a_{i, t}, \boldsymbol{f}_{-i, t}^{(m)}\right) \mid x_{i, 0}=x, f_{-i, 0}^{(m)}=f^{(m)}\right. \\
\left.\mu_{i}=\mu_{m}, \boldsymbol{\mu}_{-i}=\boldsymbol{\mu}^{(m-1)}\right] .
\end{array}
$$


We define a new player that is coupled to player $i$ in the $m$ player stochastic games defined above. We call this player the coupled player. Let $\hat{x}_{i, t}^{(m)}$ be the state of this coupled player at time $t$. The subscript $i$ and the superscript $m$ reflect the fact that this player is coupled to player $i$ in an $m$ player stochastic game. We assume that the state evolution of this player is given by:

$$
\hat{x}_{i, t+1}^{(m)} \sim \mathbf{P}\left(\cdot \mid \hat{x}_{i, t}^{(m)}, \hat{a}_{i, t}^{(m)}, f\right),
$$

where $\hat{a}_{i, t}^{(m)}=a_{i, t}^{(m)}=\mu_{m}\left(x_{i, t}^{(m)}, \boldsymbol{f}_{-i, t}^{(m)}\right)$. In other words, this coupled player takes the same action as player $i$ at every time $t$ and this action depends on the actual population state of $m-1$ players. However, note that the state evolution is dependent only on the mean field population state $f$. Let us define

$$
\hat{V}^{(m)}\left(x \mid f ; \mu_{m}, \boldsymbol{\mu}^{(m-1)}\right)=\mathbb{E}\left[\sum_{t=0}^{\infty} \beta^{t} \pi\left(\hat{x}_{i, t}^{(m)}, \hat{a}_{i, t}^{(m)}, f\right) \mid \hat{x}_{i, 0}^{(m)}=x_{0}, \hat{a}_{i, t}^{(m)}=\mu_{m}\left(x_{i, t}, \boldsymbol{f}_{-i, t}^{(m)}\right) ; \boldsymbol{\mu}^{(m-1)}\right] .
$$

Thus, $\hat{V}^{(m)}\left(x \mid f ; \mu_{m}, \mu\right)$ is the expected net present value of this coupled player, when the player's initial state is $x$, the long run average population state is $f$, and the initial population state is $f_{-i, 0}^{(m)}=f^{(m)}$. Observe that

$$
\begin{aligned}
\hat{V}^{(m)}\left(x \mid f ; \mu_{m}, \boldsymbol{\mu}^{(m-1)}\right) & \leq \sup _{\mu^{\prime} \in \mathfrak{M}} \hat{V}^{(m)}\left(x \mid f ; \mu^{\prime}, \boldsymbol{\mu}^{(m-1)}\right)=\sup _{\mu^{\prime} \in \mathfrak{M}_{O}} \hat{V}^{(m)}\left(x \mid f ; \mu^{\prime}, \boldsymbol{\mu}^{(m-1)}\right) \\
& =\tilde{V}^{*}(x \mid f)=\tilde{V}(x \mid \mu, f) .
\end{aligned}
$$

Here, the first equality follows from Lemma 2, which implies that the supremum over all cognizant strategies is the same as the supremum over oblivious strategies (since the state evolution of other players does not affect the payoff of this coupled player), and the last equality follows since $\mu \in \mathcal{P}(f)$.

Lemma 12. Let $(\mu, f)$ be a stationary equilibrium and consider an $m$ player game. Let the initial state of player $i$ be $x_{i, 0}^{(m)}=x$, and let $f^{(m)} \in \mathfrak{F}^{(m)}$ be the initial population state of $m-1$ players whose initial state is sampled independently from the distribution $f$. Assume that player $i$ uses a cognizant strategy $\mu_{m}$ and every other player uses the oblivious strategy $\mu$. Their state evolutions are given by equation (27) and (28). Also define a coupled player with initial state $\hat{x}_{i, 0}^{(m)}=x$ and let its state evolution be given by equation (30). Then, for all times $t$, and for every $y \in \mathcal{X}$, we have $\left|\operatorname{Prob}\left(\hat{x}_{i, t}^{(m)}=y\right)-\operatorname{Prob}\left(x_{i, t}^{(m)}=y\right)\right| \rightarrow 0$, almost surely $^{11}$ as $m \rightarrow \infty$.

Proof. The lemma is trivially true for $t=0$. Let us assume that it holds for all times $\tau=0,1, \cdots, t-1$.

\footnotetext{
${ }^{11}$ The almost sure convergence of the probabilities is in the randomness associated with the initial population state.
} 
Then, we have

$$
\begin{aligned}
& \operatorname{Prob}\left(x_{i, t}^{(m)}=y\right)=\sum_{z \in \mathcal{X}_{y}} \operatorname{Prob}\left(x_{i, t-1}^{(m)}=z\right) \mathbf{P}\left(y \mid z, \mu_{m}\left(z, f_{-i, t-1}^{(m)}\right), f_{-i, t-1}^{(m)}\right) \\
& \operatorname{Prob}\left(\hat{x}_{i, t}^{(m)}=y\right)=\sum_{z \in \mathcal{X}_{y}} \operatorname{Prob}\left(\hat{x}_{i, t-1}^{(m)}=z\right) \mathbf{P}\left(y \mid z, \mu_{m}\left(z, f_{-i, t-1}^{(m)}\right), f\right) .
\end{aligned}
$$

Here we use the fact that the coupled player uses the same action as player $i$ and the state evolution of the coupled player is given by equation (30). Note that the summation is over all states in the finite set $\mathcal{X}_{y}$, where $\mathcal{X}_{y}$ is defined as in equation (20).

From Lemma 10, we know that for all times $t,\left\|f_{-i, t}^{(m)}-f\right\|_{1-p} \rightarrow 0$ almost surely as $m \rightarrow \infty$. From Assumption 1, we know that the transition kernel is jointly continuous in the action $a$ and distribution $f$ (where the set of distributions $\mathfrak{F}_{p}$ is endowed with 1- $p$ norm). Since the action set is compact, this implies that for all $y, z \in \mathcal{X}, \lim _{m \rightarrow \infty} \sup _{a \in \mathcal{A}}\left|\mathbf{P}\left(y \mid z, a, f_{-i, t-1}^{(m)}\right)-\mathbf{P}(y \mid z, a, f)\right|=0$. almost surely. It follows that for every $y, z \in \mathcal{X}, \lim _{m \rightarrow \infty}\left|\mathbf{P}\left(y \mid z, \mu_{m}\left(z, f_{-i, t-1}^{(m)}\right), f_{-i, t-1}^{(m)}\right)-\mathbf{P}\left(y \mid z, \mu_{m}\left(z, f_{-i, t-1}^{(m)}\right), f\right)\right|=0$ almost surely. From the induction hypothesis, we know that for every $z \in \mathcal{X},\left|\operatorname{Prob}\left(\hat{x}_{i, t-1}^{(m)}=z\right)-\operatorname{Prob}\left(x_{i, t-1}^{(m)}=z\right)\right| \rightarrow$ 0 almost surely as $m \rightarrow \infty$. This along with the finiteness of the set $\mathcal{X}_{y}$, gives that for every $y \in \mathcal{X}$ $\left|\operatorname{Prob}\left(\hat{x}_{i, t}^{(m)}=y\right)-\operatorname{Prob}\left(x_{i, t}^{(m)}=y\right)\right| \rightarrow 0$ almost surely as $m \rightarrow \infty$. This proves the lemma.

Lemma 13. Let $(\mu, f)$ be a stationary equilibrium and consider an $m$ player game. Let the initial state of player $i$ be $x_{i, 0}^{(m)}=x$, and let $f^{(m)} \in \mathfrak{F}^{(m)}$ be the initial population state of $m-1$ players whose initial state is sampled independently from the distribution $f$. Assume that player $i$ uses a cognizant strategy $\mu_{m}$ and every other player uses the oblivious strategy $\mu$. Their state evolutions are given by equation (27) and (28). Also define a coupled player with initial state $\hat{x}_{i, 0}^{(m)}=x$ and let its state evolution be given by equation (30). Then, for all times $t$, we have $\lim \sup _{m \rightarrow \infty} \mathbb{E}\left[\pi\left(x_{i, t}^{(m)}, \mu_{m}\left(x_{i, t}^{(m)}, \boldsymbol{f}_{-i, t}^{(m)}\right), \boldsymbol{f}_{-i, t}^{(m)}\right)-\pi\left(\hat{x}_{i, t}^{(m)}, \mu_{m}\left(x_{i, t}^{(m)}, \boldsymbol{f}_{-i, t}^{(m)}\right), f\right)\right] \leq$ 0, almost surely ${ }^{12}$

Proof. Let us write $a_{i, t}^{(m)}=\mu_{m}\left(x_{i, t}^{(m)}, \boldsymbol{f}_{-i, t}^{(m)}\right)$. We have

$$
\begin{aligned}
\Delta_{i, t}^{(m)} & =\mathbb{E}\left[\pi\left(x_{i, t}^{(m)}, a_{i, t}^{(m)}, \boldsymbol{f}_{-i, t}^{(m)}\right)-\pi\left(\hat{x}_{i, t}^{(m)}, a_{i, t}^{(m)}, f\right)\right] \\
& =\mathbb{E}\left[\pi\left(x_{i, t}^{(m)}, a_{i, t}^{(m)}, \boldsymbol{f}_{-i, t}^{(m)}\right)-\pi\left(x_{i, t}^{(m)}, a_{i, t}^{(m)}, f\right)\right]+\mathbb{E}\left[\pi\left(x_{i, t}^{(m)}, a_{i, t}^{(m)}, f\right)-\pi\left(\hat{x}_{i, t}^{(m)}, a_{i, t}^{(m)}, f\right)\right] \\
& \triangleq T_{1, t}^{(m)}+T_{2, t}^{(m)} .
\end{aligned}
$$

Consider the first term. We have

\footnotetext{
${ }^{12}$ The almost sure convergence of the expected value of the payoff is in the randomness associated with the initial population state.
} 


$$
\begin{aligned}
T_{1, t}^{(m)} & \leq \sum_{y \in \mathcal{X}} \operatorname{Prob}\left(x_{i, t}^{(m)}=y\right) \sup _{a \in \mathcal{A}}\left|\pi\left(y, a, \boldsymbol{f}_{-i, t}^{(m)}\right)-\pi(y, a, f)\right| \\
& =\sum_{y \in \mathcal{X}_{x, t}} \operatorname{Prob}\left(x_{i, t}^{(m)}=y\right) \sup _{a \in \mathcal{A}}\left|\pi\left(y, a, \boldsymbol{f}_{-i, t}^{(m)}\right)-\pi(y, a, f)\right|,
\end{aligned}
$$

where the last equality follows from the fact that $x_{i, 0}^{(m)}=x$ and from equation (21). From Assumption 1 , we know that the payoff is jointly continuous in action $a$ and distribution $f$ (with the set of distributions $\mathfrak{F}_{p}$ endowed with $1-p$ norm) and the set $\mathcal{A}$ is compact. Thus, for every $y \in \mathcal{X}$, we have $\sup _{a \in \mathcal{A}}\left|\pi\left(y, a, \boldsymbol{f}_{-i, t}^{(m)}\right)-\pi(y, a, f)\right| \rightarrow 0$, almost surely as $m \rightarrow \infty$. This along with the fact that $\mathcal{X}_{x, t}$ is finite shows that $\lim \sup _{m \rightarrow \infty} T_{1, t}^{(m)} \leq 0$ almost surely.

Now consider the second term. We have

$$
\begin{aligned}
T_{2, t}^{(m)} & =\mathbb{E}\left[\pi\left(x_{i, t}^{(m)}, a_{i, t}^{(m)}, f\right)-\left(\hat{x}_{i, t}^{(m)}, a_{i, t}^{(m)}, f\right)\right] \\
& \leq \sum_{y \in \mathcal{X}}\left|\operatorname{Prob}\left(x_{i, t}^{(m)}=y\right)-\operatorname{Prob}\left(\hat{x}_{i, t}^{(m)}=y\right)\right| \sup _{a \in \mathcal{A}}|\pi(y, a, f)| \\
& =\sum_{y \in \mathcal{X}_{x, t}}\left|\operatorname{Prob}\left(x_{i, t}^{(m)}=y\right)-\operatorname{Prob}\left(\hat{x}_{i, t}^{(m)}=y\right)\right| \sup _{a \in \mathcal{A}}|\pi(y, a, f)|,
\end{aligned}
$$

where the last equality follows from the fact that $x_{i, 0}^{(m)}=\hat{x}_{i, 0}^{(m)}=x$ and from Definition 11. From Lemma 12, we know that for every $y \in \mathcal{X},\left|\operatorname{Prob}\left(x_{i, t}^{(m)}=y\right)-\operatorname{Prob}\left(\hat{x}_{i, t}^{(m)}=y\right)\right| \rightarrow 0$ almost surely $m \rightarrow \infty$. Since $\mathcal{X}_{x, t}$ is finite for every fixed $x \in \mathcal{X}$ and every time $t$, this implies that $\lim _{\sup } \rightarrow \infty T_{2, t}^{(m)} \leq 0$ almost surely. This proves the lemma.

Before we proceed further, we need one additional piece of notation. Once again let $(\mu, f)$ be a stationary equilibrium and consider an oblivious player. Let $\tilde{x}_{t}$ be the state of this oblivious player at time $t$. We assume that $\tilde{x}_{0}=x$ and since the player used the oblivious strategy $\mu$, the state evolution of this player is given by

$$
\tilde{x}_{t+1} \sim \mathbf{P}\left(\cdot \mid \tilde{x}_{t}, \tilde{a}_{t}, f\right)
$$

where $\tilde{a}_{t}=\mu\left(\tilde{x}_{t}\right)$. We let $\tilde{V}(x \mid \mu, f)$ (as defined in equation (7)) to be the oblivious value function for this player starting from state $x$.

Also, consider an $m$ player game and focus on player $i$. We represent the state of player $i$ at time $t$ by $\check{x}_{i, t}^{(m)}$. As before, the superscript $m$ on the state variable represents the fact that we are considering an $m$ player stochastic game. Let $\check{x}_{i, 0}^{(m)}=x$ and let player $i$ also use the oblivious strategy $\mu$. The initial state of every other player $j \neq i$ is drawn independently from the distribution $f$, that is, $\check{x}_{j, 0}^{(m)} \sim f$. Denote the initial distribution of all $m-1$ players (excluding player $i$ ) by $f^{(m)} \in \mathfrak{F}^{(m)}$. The state evolution of player $i$ 
is then given by

$$
\check{x}_{i, t+1}^{(m)} \sim \mathbf{P}\left(\cdot \mid \check{x}_{i, t}^{(m)}, \check{a}_{i, t}^{(m)}, \boldsymbol{f}_{-i, t}^{(m)}\right)
$$

where $\check{a}_{i, t}^{(m)}=\mu\left(\check{x}_{i, t}^{(m)}\right)$. Note that even though the player uses an oblivious strategy, its state evolution is affected by the actual population state. Let every other player $j$ also use the oblivious strategy $\mu$ and let their state evolution be given by

$$
\check{x}_{j, t+1}^{(m)} \sim \mathbf{P}\left(\cdot \mid \check{x}_{j, t}^{(m)}, \mu\left(\check{x}_{j, t}^{(m)}\right), f_{-j, t}^{(m)}\right)
$$

Define $V^{(m)}\left(x, f^{(m)} \mid \boldsymbol{\mu}^{(m)}\right)$ to be the actual value function of the player, when the initial state of the player is $x$, the initial population distribution is $f^{(m)}$ and every player uses the oblivious strategy $\mu$. That is,

$$
V^{(m)}\left(x, f^{(m)} \mid \boldsymbol{\mu}^{(m)}\right)=\mathbb{E}\left[\sum_{t=0}^{\infty} \beta^{t} \pi\left(\check{x}_{i, t}, \check{a}_{i, t}, \boldsymbol{f}_{-i, t}^{(m)}\right) \mid \check{x}_{i, 0}=x, f_{-i, 0}^{(m)}=f^{(m)} ; \mu_{i}=\mu, \boldsymbol{\mu}_{-i}=\boldsymbol{\mu}^{(m)}\right] .
$$

Lemma 14. Let $(\mu, f)$ be a stationary equilibrium and consider an $m$ player stochastic game. Let $\check{x}_{i, 0}^{(m)}=x$, and let $f^{(m)} \in \mathfrak{F}^{(m)}$ be the initial population state of $m-1$ players whose initial state is sampled independently from $f$. Assume that every player uses the oblivious strategy $\mu$ and their state evolutions are given by equations (34) and (35). Also, consider an oblivious player with $\tilde{x}_{0}=x$ and let its state evolution be given by equation (33). Then, for every time $t$ and for all $y \in \mathcal{X}$, we have $\left|\operatorname{Prob}\left(\tilde{x}_{t}=y\right)-\operatorname{Prob}\left(\check{x}_{i, t}^{(m)}=y\right)\right| \rightarrow$ 0 , almost surely as $m \rightarrow \infty$.

Proof. The lemma is trivially true for $t=0$. Let us assume that it holds for all times $\tau=0,1, \cdots, t-1$. Then, we have

$$
\begin{aligned}
\operatorname{Prob}\left(\tilde{x}_{t}=y\right) & =\sum_{z \in \mathcal{X}_{y}} \operatorname{Prob}\left(\tilde{x}_{t-1}=z\right) \mathbf{P}(y \mid z, \mu(z), f) \\
\operatorname{Prob}\left(\check{x}_{i, t}^{(m)}=y\right) & =\sum_{z \in \mathcal{X}_{y}} \operatorname{Prob}\left(\check{x}_{i, t-1}^{(m)}=z\right) \mathbf{P}\left(y \mid z, \mu(z), \boldsymbol{f}_{-i, t}^{(m)}\right) .
\end{aligned}
$$

Note that the summation above is over all states in a finite set $\mathcal{X}_{y}$ (as defined in Definition 11).

From Lemma 10, we know that for all times $t,\left\|f_{-i, t}^{(m)}-f\right\|_{1-p} \rightarrow 0$ almost surely as $m \rightarrow \infty$. From Assumption 1, we know that the transition kernel is continuous in the distribution (where the set of distributions $\mathfrak{F}_{p}$ is endowed with 1- $p$ norm). From the induction hypothesis, we know that $\left|\operatorname{Prob}\left(\tilde{x}_{t-1}=z\right)-\operatorname{Prob}\left(\check{x}_{-i, t-1}^{(m)}=z\right)\right|$ 0 . This along with the finiteness of the set $\mathcal{X}_{y}$, gives that for every $x \in \mathcal{X}$

$$
\left|\operatorname{Prob}\left(\tilde{x}_{t}=x\right)-\operatorname{Prob}\left(\check{x}_{i, t}^{(m)}=x\right)\right| \rightarrow 0
$$

almost surely as $m \rightarrow \infty$. This proves the lemma. 
Lemma 15. Let $(\mu, f)$ be a stationary equilibrium and consider an m player stochastic game. Let $\check{x}_{i, 0}^{(m)}=$ $x$, and let $f^{(m)} \in \mathfrak{F}^{(m)}$ be the initial population state of $m-1$ players whose initial state is sampled independently from $f$. Assume that every player uses the oblivious strategy $\mu$ and their state evolutions are given by equations (34) and (35). Also, consider an oblivious player with $\tilde{x}_{0}=x$ and let its state evolution be given by equation (33). Then for all times $t$, we have $\mathbb{E}\left[\pi\left(\tilde{x}_{t}, \mu\left(\tilde{x}_{t}\right), f\right)-\pi\left(\check{x}_{i, t}^{(m)}, \mu\left(\check{x}_{i, t}^{(m)}\right), \boldsymbol{f}_{-i, t}^{(m)}\right)\right] \rightarrow$ 0 , almost surely as $m \rightarrow \infty$.

Proof. Define $\Delta_{i, t}^{(m)}$ as

$$
\begin{aligned}
\Delta_{i, t}^{(m)} & =\mathbb{E}\left[\pi\left(\tilde{x}_{t}, \mu\left(\tilde{x}_{t}\right), f\right)-\pi\left(\check{x}_{i, t}^{(m)}, \mu\left(\check{x}_{i, t}^{(m)}\right), \boldsymbol{f}_{-i, t}^{(m)}\right)\right] \\
& =\mathbb{E}\left[\pi\left(\tilde{x}_{t}, \mu\left(\tilde{x}_{t}\right), f\right)-\pi\left(\tilde{x}_{t}, \mu\left(\tilde{x}_{t}\right), \boldsymbol{f}_{-i, t}^{(m)}\right)\right]+\mathbb{E}\left[\pi\left(\tilde{x}_{t}, \mu\left(\tilde{x}_{t}\right), \boldsymbol{f}_{-i, t}^{(m)}\right)-\pi\left(\check{x}_{i, t}^{(m)}, \mu\left(\check{x}_{i, t}^{(m)}\right), \boldsymbol{f}_{-i, t}^{(m)}\right)\right] \\
& \triangleq T_{1, t}^{(m)}+T_{2, t}^{(m)} .
\end{aligned}
$$

Note that from Lemma 10, we have that $\left\|f_{-i, t}^{(m)}-f\right\|_{1-p} \rightarrow 0$ almost surely as $m \rightarrow \infty$. From Assumption 1 , we know that the payoff is continuous in the distribution, where the set of distributions $\mathfrak{F}_{p}$ is endowed with 1-p norm. Thus, for every $y$ and $a$, we have

$$
\left|\pi(y, a, f)-\pi\left(y, a, \boldsymbol{f}_{-i, t}^{(m)}\right)\right| \rightarrow 0
$$

as $m \rightarrow \infty$. Consider the first term. We have:

$$
\begin{aligned}
T_{1, t}^{(m)} & =\sum_{y \in \mathcal{X}} \operatorname{Prob}\left(\tilde{x}_{t}=y\right)\left|\pi(y, \mu(y), f)-\pi\left(y, \mu(y), \boldsymbol{f}_{-i, t}^{(m)}\right)\right| \\
& =\sum_{y \in \mathcal{X}_{x, t}} \operatorname{Prob}\left(\tilde{x}_{t}=y\right)\left|\pi(y, \mu(y), f)-\pi\left(y, \mu(y), \boldsymbol{f}_{-i, t}^{(m)}\right)\right|,
\end{aligned}
$$

where the last equality follows from the fact that $\tilde{x}_{0}=x$ and from Definition 11 . Since $\mathcal{X}_{x, t}$ is a finite set for every initial state $x \in \mathcal{X}$ and every time $t$, we get that $T_{1, t}^{(m)} \rightarrow 0$ almost surely as $m \rightarrow \infty$.

Consider now the second term. We have:

$$
\begin{aligned}
T_{2, t}^{(m)} & =\mathbb{E}\left[\pi\left(\tilde{x}_{t}, \mu\left(\tilde{x}_{t}\right), \boldsymbol{f}_{-i, t}^{(m)}\right)-\pi\left(\check{x}_{i, t}^{(m)}, \mu\left(\check{x}_{i, t}^{(m)}\right), \boldsymbol{f}_{-i, t}^{(m)}\right)\right] \\
& =\sum_{y \in \mathcal{X}} \operatorname{Prob}\left(\tilde{x}_{t}=y\right) \pi\left(y, \mu(y), \boldsymbol{f}_{-i, t}^{(m)}\right)-\sum_{y \in \mathcal{X}} \operatorname{Prob}\left(\check{x}_{i, t}^{(m)}=y\right) \pi\left(y, \mu(y), \boldsymbol{f}_{-i, t}^{(m)}\right) \\
& =\sum_{y \in \mathcal{X}_{t}}\left(\operatorname{Prob}\left(\tilde{x}_{t}=y\right)-\operatorname{Prob}\left(\check{x}_{i, t}^{(m)}=y\right)\right) \pi\left(y, \mu(y), \boldsymbol{f}_{-i, t}^{(m)}\right) .
\end{aligned}
$$

From Lemma 14, equation (37), and the finiteness of $\mathcal{X}_{x, t}$, we get that $\lim _{\sup } \rightarrow \infty T_{2, t}^{(m)} \leq 0$ almost surely. This proves the lemma.

Proof of Theorem 2. Let us define

$$
\Delta V^{(m)}\left(x, f^{(m)}\right) \triangleq V^{(m)}\left(x, f^{(m)} \mid \mu_{m}, \boldsymbol{\mu}^{(m-1)}\right)-V^{(m)}\left(x, f^{(m)} \mid \boldsymbol{\mu}^{(m)}\right) .
$$


Then we need to show that for all $x, \limsup _{m \rightarrow \infty} \Delta V^{(m)}\left(x, f^{(m)}\right) \leq 0$ almost surely. We can write

$$
\begin{aligned}
& \Delta V^{(m)}\left(x, f^{(m)}\right)=V^{(m)}\left(x, f^{(m)} \mid \mu_{m}, \boldsymbol{\mu}^{(m-1)}\right)-\tilde{V}(x \mid \mu, f)+\tilde{V}(x \mid \mu, f)-V^{(m)}\left(x, f^{(m)} \mid \boldsymbol{\mu}^{(m)}\right) \\
& \quad \leq V^{(m)}\left(x, f^{(m)} \mid \mu_{m}, \boldsymbol{\mu}^{(m-1)}\right)-\hat{V}^{(m)}\left(x \mid f ; \mu_{m}, \boldsymbol{\mu}^{(m-1)}\right)+\tilde{V}(x \mid \mu, f)-V^{(m)}\left(x, f^{(m)} \mid \boldsymbol{\mu}^{(m)}\right) \\
& \quad \triangleq T_{1}^{(m)}+T_{2}^{(m)} .
\end{aligned}
$$

Here the inequality follows from equation (32). Consider the term $T_{1}^{(m)}$. We have

$$
\begin{aligned}
T_{1}^{(m)} & =V^{(m)}\left(x, f^{(m)} \mid \mu_{m}, \boldsymbol{\mu}^{(m-1)}\right)-\hat{V}^{(m)}\left(x \mid f ; \mu_{m}, \boldsymbol{\mu}^{(m-1)}\right) \\
& =\mathbb{E}\left[\sum_{t=0}^{\infty} \beta^{t}\left(\pi\left(x_{i, t}^{(m)}, a_{i, t}^{(m)}, \boldsymbol{f}_{-i, t}^{(m)}\right)-\pi\left(\hat{x}_{i, t}^{(m)}, \hat{a}_{i, t}^{(m)}, f\right)\right)\right],
\end{aligned}
$$

where the last equality follows from equations (29) and (31). Note that $x_{i, 0}=\hat{x}_{i, 0}=x$ and $a_{i, t}=$ $\hat{a}_{i, t}=\mu_{m}\left(x_{i, t}, \boldsymbol{f}_{-i, t}^{(m)}\right)$ and the state transitions of players are given by equations (27), (28), and (30). From Lemma 13, we have $\limsup _{m \rightarrow \infty} \mathbb{E}\left[\sum_{t=0}^{T-1} \beta^{t}\left(\pi\left(x_{i, t}^{(m)}, a_{i, t}^{(m)}, \boldsymbol{f}_{-i, t}^{(m)}\right)-\pi\left(\hat{x}_{i, t}^{(m)}, \hat{a}_{i, t}^{(m)}, f\right)\right)\right] \leq 0$, almost surely for any finite time $T$. From Lemma 1, we have, almost surely

$$
\mathbb{E}\left[\sum_{t=T}^{\infty} \beta^{t}\left(\pi\left(x_{i, t}^{(m)}, \hat{a}_{i, t}^{(m)}, \boldsymbol{f}_{-i, t}^{(m)}\right)-\pi\left(\hat{x}_{i, t}^{(m)}, a_{i, t}^{(m)}, f\right)\right)\right] \leq 2 C(x, T),
$$

which goes to zero as $T \rightarrow \infty$. This proves that $\limsup _{m \rightarrow \infty} T_{1}^{(m)} \leq 0$ almost surely. Similar analysis (with an application of Lemma 15) shows that $\lim \sup _{m \rightarrow \infty} T_{2}^{(m)} \leq 0$ almost surely, yielding the result.

Proof of Theorem 3. Similar to the proof of Theorem 2, let us define

$$
\Delta V^{(m)}\left(x, f^{(m)}\right) \triangleq V^{(m)}\left(x, f^{(m)} \mid \mu_{m}, \boldsymbol{\mu}^{(m-1)}\right)-V^{(m)}\left(x, f^{(m)} \mid \boldsymbol{\mu}^{(m)}\right) .
$$

Then we need to show that for all $x, \lim _{\sup _{m \rightarrow \infty}} \Delta V^{(m)}\left(x, f^{(m)}\right) \leq 0$ almost surely. We can write

$$
\begin{aligned}
\Delta V^{(m)}\left(x, f^{(m)}\right) & =V^{(m)}\left(x, f^{(m)} \mid \mu_{m}, \boldsymbol{\mu}^{(m-1)}\right)-\tilde{V}(x \mid \mu, f)+\tilde{V}(x \mid \mu, f)-V^{(m)}\left(x, f^{(m)} \mid \boldsymbol{\mu}^{(m)}\right) \\
& \leq V^{(m)}\left(x, f^{(m)} \mid \mu_{m}, \boldsymbol{\mu}^{(m-1)}\right)-\hat{V}^{(m)}\left(x \mid f ; \mu_{m}, \boldsymbol{\mu}^{(m-1)}\right)+\tilde{V}(x \mid \mu, f)-V^{(m)}\left(x, f^{(m)} \mid \boldsymbol{\mu}^{(m)}\right) \\
& \triangleq T_{1}^{(m)}+T_{2}^{(m)}
\end{aligned}
$$

where $\hat{V}^{(m)}$ is defined as in (31) (and in particular, using the limit profit function $\pi$ ). Here the inequality 
follows from equation (32). Consider the term $T_{1}^{(m)}$. We have

$$
\begin{aligned}
T_{1}^{(m)} & =V^{(m)}\left(x, f^{(m)} \mid \mu_{m}, \boldsymbol{\mu}^{(m-1)}\right)-\hat{V}^{(m)}\left(x \mid f ; \mu_{m}, \boldsymbol{\mu}^{(m-1)}\right) \\
& =\mathbb{E}\left[\sum_{t=0}^{\infty} \beta^{t}\left(\pi_{m}\left(x_{i, t}^{(m)}, a_{i, t}^{(m)}, \boldsymbol{f}_{-i, t}^{(m)}\right)-\pi\left(\hat{x}_{i, t}^{(m)}, \hat{a}_{i, t}^{(m)}, f\right)\right)\right],
\end{aligned}
$$

where the last equality follows from equation (29) (with $\pi$ replaced by $\pi_{m}$ ) and equation (31). Note that $x_{i, 0}=\hat{x}_{i, 0}=x$ and $a_{i, t}=\hat{a}_{i, t}=\mu_{m}\left(x_{i, t}, \boldsymbol{f}_{-i, t}^{(m)}\right)$ and the state transitions of players are given by equations (27), (28), and (30). Now,

$T_{1}^{(m)}=\mathbb{E}\left[\sum_{t=0}^{\infty} \beta^{t}\left(\pi_{m}\left(x_{i, t}^{(m)}, a_{i, t}^{(m)}, \boldsymbol{f}_{-i, t}^{(m)}\right)-\pi_{m}\left(\hat{x}_{i, t}^{(m)}, \hat{a}_{i, t}^{(m)}, f\right)\right)+\left(\pi_{m}\left(\hat{x}_{i, t}^{(m)}, \hat{a}_{i, t}^{(m)}, f\right)-\pi\left(\hat{x}_{i, t}^{(m)}, \hat{a}_{i, t}^{(m)}, f\right)\right)\right]$,

Using equicontinuity and the uniform growth rate bound, a similar argument to the proof of Theorem 2 (via Lemmas 13 and 15) shows that:

$$
\limsup _{m \rightarrow \infty} \mathbb{E}\left[\sum_{t=0}^{\infty} \beta^{t}\left(\pi_{m}\left(x_{i, t}^{(m)}, a_{i, t}^{(m)}, \boldsymbol{f}_{-i, t}^{(m)}\right)-\pi_{m}\left(\hat{x}_{i, t}^{(m)}, \hat{a}_{i, t}^{(m)}, f\right)\right)\right] \leq 0
$$

almost surely. Recall that, for all $x, a, f, \lim _{m \rightarrow \infty} \pi_{m}(x, a, f)=\pi(x, a, f)$. Since $\mathcal{A}$ is compact and increments are bounded, it follows that $\pi_{m}\left(\hat{x}_{i, t}^{(m)}, \hat{a}_{i, t}^{(m)}, f\right)-\pi\left(\hat{x}_{i, t}^{(m)}, \hat{a}_{i, t}^{(m)}, f\right) \rightarrow 0$ almost surely as $m \rightarrow$ $\infty$, for all times $t$. Using the fact that increments are bounded, the uniform growth rate bound, and the dominated convergence theorem, the expectation of the preceding difference also approaches zero almost surely. Finally, by truncating the sum at time $T$, an argument similar to the proof of Theorem 2 gives:

$$
\limsup _{m \rightarrow \infty} \mathbb{E}\left[\sum_{t=0}^{\infty} \beta^{t}\left(\pi_{m}\left(\hat{x}_{i, t}^{(m)}, \hat{a}_{i, t}^{(m)}, f\right)-\pi\left(\hat{x}_{i, t}^{(m)}, \hat{a}_{i, t}^{(m)}, f\right)\right)\right] \leq 0
$$

This proves that $\lim \sup _{m \rightarrow \infty} T_{1}^{(m)} \leq 0$ almost surely. Similar analysis shows that $\lim _{\sup } \operatorname{sim}_{2} T_{2}^{(m)} \leq 0$ almost surely, yielding the result. 